August 1999 • NREL/BK-590-26952

\title{
Workshop on Basic Research Opportunities in Photovoltaics
}

Editors: John Benner, Satyen Deb, and Robert McConnell

Held in Conjunction with the 195th Meeting of the Electrochemical Society

Seattle, Washington

May 3, 1999

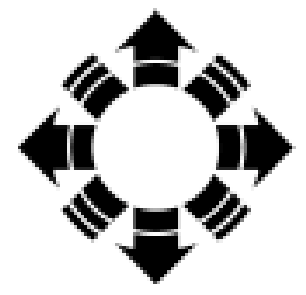

National Renewable Energy Laboratory

1617 Cole Boulevard

Golden, Colorado 80401-3393

NREL is a U.S. Department of Energy Laboratory

Operated by Midwest Research Institute $\bullet$ Battelle $\bullet$ Bechtel

Contract No. DE-AC36-98-G010337 

August $1999 \quad \cdot \quad$ NREL/BK-590-26952

\title{
Workshop on Basic Research Opportunities in Photovoltaics
}

\author{
Editors: John Benner, Satyen Deb, and \\ Robert McConnell
}

Held in Conjunction with the 195th Meeting of the Electrochemical Society

Seattle, Washington

May 3, 1999

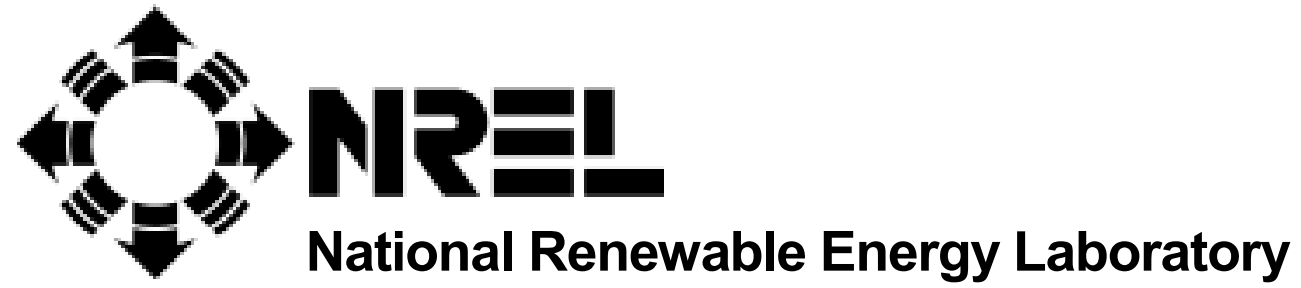

1617 Cole Boulevard

Golden, Colorado 80401-3393

NREL is a U.S. Department of Energy Laboratory

Operated by Midwest Research Institute $\bullet$ Battelle $\bullet$ Bechtel

Contract No. DE-AC36-98-G010337 


\section{NOTICE}

This report was prepared as an account of work sponsored by an agency of the United States government. Neither the United States government nor any agency thereof, nor any of their employees, makes any warranty, express or implied, or assumes any legal liability or responsibility for the accuracy, completeness, or usefulness of any information, apparatus, product, or process disclosed, or represents that its use would not infringe privately owned rights. Reference herein to any specific commercial product, process, or service by trade name, trademark, manufacturer, or otherwise does not necessarily constitute or imply its endorsement, recommendation, or favoring by the United States government or any agency thereof. The views and opinions of authors expressed herein do not necessarily state or reflect those of the United States government or any agency thereof.

Available to DOE and DOE contractors from:

Office of Scientific and Technical Information (OSTI)

P.O. Box 62

Oak Ridge, TN 37831

Prices available by calling 423-576-8401

Available to the public from:

National Technical Information Service (NTIS)

U.S. Department of Commerce

5285 Port Royal Road

Springfield, VA 22161

$703-605-6000$ or $800-553-6847$

or

DOE Information Bridge

http://www.doe.gov/bridge/home.html

Printed on paper containing at least $50 \%$ wastepaper, including $20 \%$ postconsumer waste 


\section{TABLE OF CONTENTS}

Basic Research Opportunities in Photovoltaics Workshop

J. Benner, S. Deb, and R.D. McConnell 1

Research Opportunities in Crystalline Silicon Photovoltaics for the $21^{\text {st }}$ Century

H.A. Atwater, B. Sopori, T. Ciszek, L.C. Feldman, J. Gee, and A. Rohatgi

Amorphous and Microcrystalline Silicon Solar Cells

S. Wagner, D.E. Carlson, and H.M. Branz.

Basic Research Opportunities in Cu-Chalcopyrite Photovoltaics

A. Rockett, R.N. Bhattacharya, C. Eberspacher, V. Kapur, and S.-H Wei

Critical Issues and Research Needs for CdTe-Based Solar Cells

A.D. Compaan, J.R. Sites, R.W. Birkmire, C.S. Ferekides, and A.L. Fahrenbruch ...43

Next Generation Thin Films for Photovoltaics: InGaAsN

E.D. Jones, A.A. Allerman, D.J. Friedman, J.F. Geisz, J.F. Klem, S.R. Kurtz,

N.R. Modine, W. Shan, C. Tu, and W. Walukiewicz .55

Novel Materials for Photovoltaic Technologies

P. Alivisatos, S. Carter, D. Ginley, G. Meyer, A. Nozik, and S. Rosenthal.

Transparent Conducting Oxides: Status and Opportunities in Basic Research

T.J. Coutts, T.O. Mason, J.D. Perkins, and D.S. Ginley .. .77

Photovoltaics Characterization: An Overview

Y.H. Kao, L. Kazmerski, K.G. Lynn, and A. Mascarenhas 


\title{
BASIC RESEARCH OPPORTUNITIES IN PHOTOVOLTAICS WORKSHOP
}

\author{
J. Benner, S. Deb, and R.D. McConnell \\ National Renewable Energy Laboratory \\ Golden, CO 80401-3393
}

\section{Preface and Executive Summary}

Photovoltaic (PV) technology for conversion of sunlight to electricity is the most cost-effective method for meeting the electric power needs of many consumers around the world today. This is due in large part to more than 25 years of research and development supported by nations throughout the world with the vision to provide a clean, renewable source of energy. The U.S. PV research program was by far the largest during the first decade of development. U.S. based manufacturers today hold the major market share of this successful, rapidly growing worldwide business that generated nearly a billion dollars in sales during 1998. Most of the product serves applications some distance from the utility power grid or in installations that compete with retail prices for electricity. Consistent with the time horizon needed for any major change in national infrastructure, another 25 years of sustained, aggressive growth will be required for PV to displace a significant fraction of conventional energy generation sources. This growth will rely on continuous introduction of new technology, underpinned by sound fundamental research. As we enter this phase, unfortunately, U.S. programs lag fundamental research efforts in Europe and Japan in both scope and funding.

As we embark on this phase of development, both the National PV Program within the DOE Office of Energy Efficiency and Renewable Energy and Basic Energy Science within the Office of Science are focusing increasing attention on the basic research issues that must be addressed to maintain the PV growth rate as well as on opportunities to accelerate this pace. The Basic Research Opportunities in Photovoltaics Workshop, held May 3, 1999 in Seattle, Washington, brought together experts in PV and related fields to offer guidance for initiatives on high payoff research programs.

The expediency of a one-day meeting was made possible by a number of factors. First, this workshop built on the findings of a prior meeting. A Research Assistance Task Force, chaired by Alex Zunger, met during July 27-29, 1992 to discuss Research Opportunities in Photovoltaic Semiconductors. These extended interactions were documented in a special issue of the Journal of Electronic Materials (Volume 22, number 1, January 1993). Second, the $195^{\text {th }}$ Meeting of the Electrochemical Society, which included a special Symposium focused on photovoltaics, provided a venue which attracted many of workshop participants such that costs and inconvenience of travel could be minimized. Finally, and most importantly, the workshop participants carried out extensive dialogue by phone, fax, and e-mail prior to arriving on May 3 and after the meeting as they identified their key research issues and prepared the final manuscripts.

The workshop was structured into eight topics. Each topic area opened with a presentation in which the participants were asked to address the following areas:

- A brief introduction of the area of research

- Key research issues that were identified in the previous workshop of July 1992

- What fundamental research has been done since then or is currently being done to address those issues

- What are the research issues that are still relevant in light of advances made since the first workshop

- Identification of new fundamental research opportunities that will lead to important advances and innovations 
- Identification of significant commonalties and common research issues that have a crosscutting impact such as logically exist in silicon-based thin films, II-VI and related materials.

Participants in each topic area broke out into separate discussion groups to develop a concise set of issues and opportunities in light of their own views along with input from questions and comments from the other workshop participants that followed the presentation. Many of the attendees roved between groups to share in discussion of several topics. Results of the discussions were captured on flip-charts for presentation to the entire workshop as we reconvened at the end of the day.

After the meeting, participants in each working topic continued discussions by electronic means completing journal articles which are to be published as a separate section in the ECS Proceedings of the PV for the $21^{\text {st }}$ Centurv Sumposium.

For executive review, the key issues and opportunities identified by the topic area participants are highlighted below. These are not necessarily in order of priority.

Amorphous and micro-crystalline silicon (these three bullets are in priority order)

- Understanding and control of Si and alloy film structure, with increasing emphasis on high deposition rate as the structural order increases.

- Understand the role of $\mathrm{H}$ in establishing nanostructure, in alloying and doping, in metastability, and as a structural modifier during solar cell operation.

- Understand and control the gas phase chemistry, the reactions on the growing surface, and their effects on device properties

Crystalline Silicon

- Fundamentals of impurities and defects

- Interface and passivation issues for screen-printed contacts

- Impurity separation technology

- Thin-layer crystalline silicon of high quality, deposited at high growth rate using either lowtemperature processing or a low-cost substrate compatible with high temperature processing

- Control of light - texturing, light trapping, and optical modeling

Cadmium Telluride

- Understand the basic nature of polycrystalline CdTe needed for truly predictive models, alternative process pathways, and meaningful process monitors and control.

- Measurements on samples prepared with systematic variation of process variables and incorporation of extrinsic dopants to elucidate the role of impurity atoms $(\mathrm{Cl}, \mathrm{O}, \mathrm{Cu})$ and defects on bulk CdTe properties and nanoscale variations of the polycrystalline material and its related alloys

- CdS/CdTe junction modeling and analysis with controlled degree of interdiffusion and other process variables.

- Transparent conducting oxide front layers and their impact on subsequent depositions.

- Understanding the role of $\mathrm{Cu}$ in back contacts and exploration of $\mathrm{Cu}$-free contact

Copper Indium Diselenide

- Development of an integrated predictive understanding of CIGSS materials and devices

- Development of novel deposition techniques and characterization of mechanisms of growth in existing and novel processes

- Novel materials, especially with wide energy gaps $(>1.7 \mathrm{eV})$ other than CIGSS alloys 
- Development of real-time material characterization for process control

- Alternative front and rear contact materials

III-V Materials

- Investigation of the influence of InGaAsN growth conditions on materials properties such as background impurity incorporation, carrier trap density and energies, and minority carrier properties.

- Fundamental properties of $1 \mathrm{eV}$ semiconductors for high efficiency, multiple junction devices.

- Details transport studies in compensated InGaAsN to determine if electron localization is intrinsic to InGaAsN

- Development of technology capable of measuring I-V performance of multiple junction device.

Novel Materials and Energy Conversion Approaches

- Nano/molecular composites - hierarchical structures

- Organic semiconductors

- Hot carrier devices

Semiconducting Oxides

- Synthesis, characterization, and understanding of new compositions and phases of TCOs

- Investigate scattering mechanisms to guide development of TCOs with higher electron mobilities

- Investigate the role of impurities and defects

- All oxide devices

Characterization

- Atomic and nanoscale characterization of impurity, native defects, extended defects, and interfaces - higher spatial resolution and trace impurities

- Performance characterization of developing technologies - high flux operation, multijunction, lower bandgap

- In-situ probes for diagnostics and process control - immediate response, integrated to ensure relevance 


\title{
RESEARCH OPPORTUNITIES IN CRYSTALLINE SILICON PHOTOVOLTAICS FOR THE $21^{\text {ST }}$ CENTURY
}

\author{
Harry A. Atwater \\ Thomas J. Watson Laboratory of Applied Physics \\ California Institute of Technology, Pasadena, CA 91125 \\ Bhushan Sopori and Ted Ciszek \\ National Renewable Energy Laboratory \\ Golden, CO 80401-3393 \\ Leonard C. Feldman \\ Department of Physics and Astronomy \\ Vanderbilt University \\ Nashville, TN 37235 \\ James Gee \\ Sandia National Laboratories \\ Albuquerque, NM 87185-0752 \\ Ajeet Rohatgi \\ School of Electrical Engineering \\ Georgia Institute of Technology \\ Atlanta, GA 30332
}

\begin{abstract}
Crystalline silicon continues to be the dominant semiconductor material used for terrestrial photovoltaics. This paper discusses the scientific issues associated with silicon photovoltaics processing and cell design that may yield cell and module performance improvements, both evolutionary and revolutionary in nature. We first survey critical issues in "thick" crystalline silicon photovoltaics, including novel separations processes for impurity removal, impurity and defect fundamentals, interface passivation, the role of hydrogen, and high-throughput, kinetically-limited materials processing. Second, we outline emerging opportunities for creation of a very different "thin-layer" silicon cell structure, including the scientific issues and engineering challenges associated with thinlayer silicon processing and cell design.
\end{abstract}

\section{INTRODUCTION}

Today's basic research advances in materials physics and materials synthesis and processing will provide the foundation for a large-scale industrial photovoltaics technology that appears likely to develop over the next 10-30 years. Over this time frame, the photovoltaics industry is expected to expand to a production level on the order of 10's GW/year worldwide, at which point it will be able to provide an important global source of clean energy. In this future, a prototypical photovoltaic manufacturing facility may be anticipated to produce on the order of $1 \mathrm{GW} /$ year - and by simple considerations one can project for example that such a plant will need to achieve a throughput on the order of 10 $\mathrm{m}^{2}$ of modules per minute. 
Crystalline silicon is very likely to maintain a quite significant role in photovoltaics technology over this time frame. Indeed, between 1992 and 1998, crystalline silicon has expanded its market share from $73 \%$ to $86 \%$ of the market relative to other photovoltaics technologies. Shipments of crystalline Si photovoltaics amounted to $132 \mathrm{MW}$ per annum by 1998 , and currently the Si photovoltaics industry is growing faster than its large cousin the microelectronics industry. Because of this large and continuing investment in silicon photovoltaics, it will be critical to address fundamental materials physics and materials synthesis issues related to crystalline silicon photovoltaics, since these basic research investments may enable further efficiency improvements and cost-reductions to occur.

Several significant scientific, technical and economic advantages accrue to crystalline $\mathrm{Si}:$

- Its device physics and materials physics issues are better understood than competing device materials. However, very important basic materials physics issues remain outstanding for crystalline silicon photovoltaics; critical issues related to minority carrier lifetime enhancement in multicrystalline or polycrystalline silicon are unlikely to be addressed by the microelectronics industry in the future.

- It is a serendipitous materials system: it has an extremely useful native oxide in $\mathrm{SiO}_{2}$; as an elemental material, it lacks stoichiometry problems; dopants such as $\mathrm{Al}$ and $\mathrm{P}$ can also play a role in gettering; silicon is mechanically robust relative to other semiconductors, facilitating cell processing.

- Salubrity: it is a non-toxic, plentiful (and indeed even a nondepletable) material.

- There exists already a market-proven industrial manufacturing infrastructure.

Nevertheless, a crystalline silicon photovoltaics industry with GW/year-scale plants will likely look very different from today's technology. Cells and modules will need to be much easier to manufacture while maintaining high efficiency, processes will have much higher throughputs, and devices and processes will have significantly reduced material and energy inputs and reduced waste streams. High-throughput processing will be critical importance. The silicon photovoltaics industry has traditionally adapted processes from the silicon microelectronics industry for manufacturing. The high throughputs required for the future $\mathrm{GW} /$ year-scale plants will require manufacturing paradigms more closely resembling other industries with similar processing throughputs, such as petrochemicals, plastics or glass products. Basic research opportunities exist for adapting high-throughput processes for crystalline-silicon photovoltaic cell and module manufacturing, developing new cell and module designs that are more amenable to highthroughput processing, development of fundamental process models to help guide research, and development of in-situ process monitors for better control and optimization of processes. More rapid processing of silicon will also require a more thorough understanding of the silicon material, principally the effects of defects and impurities. Research in fundamentals of gettering, passivation, precipitate formation and dissolution will be necessary. As the silicon materials demand and consumption of the photovoltaics industry grow beyond that which can be satisfied at the margins of silicon production for microelectronics, new methods of synthesis and purification of solar-grade silicon feedstock will be required. Also needed are more productive, less material consumptive methods for producing silicon substrates. In particular, thin substrates can reduce material consumption and improve manufacturing productivity. However, thin substrates offer a number of challenges for fundamental research, including advanced surface and contact 
passivation, mechanical strength of thin substrates, and enhancement of optical absorption.

At the same time, significant research and manufacturing investments are also being made in thin-film photovoltaic materials, such as amorphous silicon, CdTe, and copper indium diselenide and related chalcopyrite semiconductors. This is at least partly motivated by the potential manufacturing cost reductions that accompany processing of large, module-size sheets of absorber material rather than individual cells. For this reason, it is interesting to explore the possibility of a "thin layer" (1-30 $\mu \mathrm{m}$ thick) polycrystalline silicon cell or even perhaps a polycrystalline silicon/amorphous silicon tandem structure may compete favorably with the other thin film technologies.

Thus, we can anticipate two kinds of basic research opportunities in crystalline silicon photovoltaics:

1. Rapid Evolutionary: those that enable a rapid evolution from today's cell designs and industrial processes to those that can sustain manufacturing plants with GW/year throughputs. Critical areas include impurity separation from Si feedstock, impurity and defect effects on cell performance, interface passivation issues at contacts, and the role of hydrogen. These are all currently issues facing Si photovoltaics, but they will have to be addressed anew in the future in the context of very high throughput manufacturing.

2. Revolutionary: those that enable a new silicon photovoltaics technology to be developed based on thin-layer polycrystalline silicon growth in sheet form on low-cost substrates, most likely with very different cell designs and materials processing than that used in "thick" crystalline silicon photovoltaics today. This is currently a high-risk approach, since many uncertainties exist about cell structure and silicon processing approaches.

\section{IMPURITY REMOVAL FROM SILICON FEEDSTOCK}

The starting silicon for both photovoltaics and semiconductor integrated circuit applications is $99 \%$ pure metallurgical-grade (MG) $\mathrm{Si}$. Integrated circuit industry chlorosilane purification and deposition steps increase the purity to more than adequate levels for photovoltaic use, but also increase the cost unacceptably. So the $\mathrm{Si}$ photovoltaics industry has been using reject material from integrated circuit polysilicon and single-crystal production. But as production techniques improve and as the $\mathrm{Si}$ photovoltaics industry grows ( $\sim 30 \%$ per annum) at a faster rate than the integrated circuit industry, this material becomes rarer and more expensive. New sources of polysilicon will be needed [1,2]. Demand first exceeded supply in 1996. The present downturn in the integrated circuit industry has temporarily relieved the photovoltaics feedstock shortage, but projections by one of the largest polysilicon manufacturers indicate that demand for reject silicon will exceed the supply by a factor of 2 to 4 within 10 years. This does not represent a fundamental material shortage problem, since the technology, quartzite, and coke needed to make feed stock is in abundant supply. The issue is to supply feedstock with necessary, but only sufficient, purity at an acceptable cost. 
Figure 1. Boron depth profiles at the surface of MG-Si subjected to various surface and annealing treatments. All treatments that included porous $\mathrm{Si}$ etching (inset) resulted in preferential accumulation of $B$ at the surface [8].

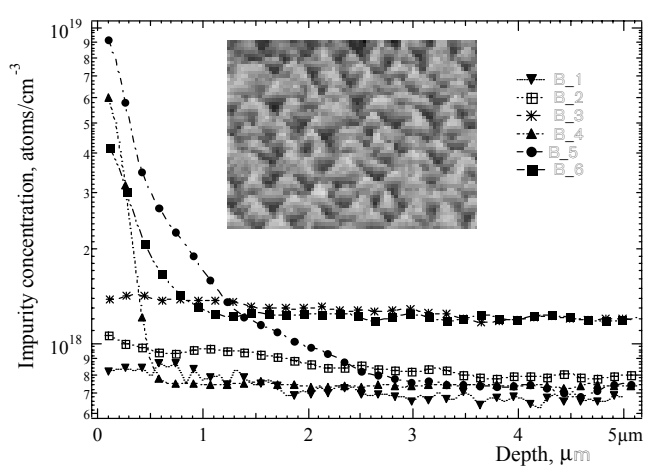

The trichlorosilane $\left(\mathrm{SiHCl}_{3}\right)$ distillation and reduction method is used for over $95 \%$ of polysilicon production, but is very energy intensive, and it produces large amounts of waste, including a mix of environmentally damaging chlorinated compounds and about $80 \%$ of the initial MG-Si material. In addition, the feedstock produced by this method far exceeds the following preferred purity requirements of the photovoltaics industry: either $\mathrm{B}$ or $\mathrm{P}$ doping, with no compensation; resistivity at $25^{\circ} \mathrm{C}$ should be greater than $1 \mathrm{ohm}-$ $\mathrm{cm}$; oxygen and carbon should not exceed the saturation limits in the melt; and the total non-dopant impurity concentration should be less than 1 ppma [3].

Fresh approaches are needed to originate novel separation technologies that can extract $\mathrm{B}, \mathrm{Al}$, transition metal impurities and other impurities from impure silicon, to meet the purity requirements listed above, but in a simpler process. Some examples of new approaches that are in early stages of investigation include:

(i) use of electron beam and plasma treatments to remove impurities from MG$\mathrm{Si}[4]$.

(i) directly purifying granular MG-Si using repeated porous silicon etching, subsequent annealing, and surface impurity removal, as illustrated in Fig. 1 [5].

(ii) a method that uses MG-Si and absolute alcohol as the starting materials [6].

(iii) gaseous treatments of MG Si melts guided by thermochemical calculations [7].

(iv) use of impurity partitioning when silicon is recrystallized from MG Si/metal eutectic systems [8].

Approaches like these or other yet-to-be-determined innovative methods could have a major impact on the continued success and growth of the Si photovoltaics industry if one is discovered that is intrinsically simpler than current technology, yet yields adequate $\mathrm{Si}$ purity.

\section{IMPURITIES AND DEFECTS IN PHOTOVOLTAIC-SI}

The silicon photovoltaics industry uses low-cost substrates, which contain high concentrations of impurities and defects. In recent years, a variety of measures such as higher quality feedstock and better crucible quality have resulted in reduced metallic impurity content to the levels approaching $10^{14} \mathrm{~cm}^{-3}$ while carbon and oxygen 
concentrations remain below saturation levels. Furthermore, there are ongoing attempts to improve the thermal conditions during the crystal growth processes that have yielded substrates with very low-average defect density-typically $<10^{5} / \mathrm{cm}^{2}$. However, as the defect density reduces, the defects have a tendency to form clusters [9].

Figure 1 is a map showing the distribution of defects in a $25 \mathrm{~cm}^{2}$ section of a commercial, multicrystalline silicon (mc-Si) wafer. The darker regions indicate higher defect densities. This figure shows that a majority of the wafer has a low or zero dislocation density, while other regions have high concentrations of defects that are clustered together. Although the average value of the dislocation density in the entire wafer is about $10^{5} / \mathrm{cm}^{2}$, defect clusters can be seen where the local defect density can exceed $10^{7} / \mathrm{cm}^{2}$. Detailed analyses show that a defect cluster involves a series of long, intertwined dislocation loops. Because these loops and networks are high-energy defect configurations, they are thermally unstable and can change during device processing. Furthermore, the defect clusters can be efficient nucleation sites that can become decorated with impurity precipitates during crystal growth. This propensity for impurity decoration of a defect cluster has a strong bearing on how it affects the device performance.

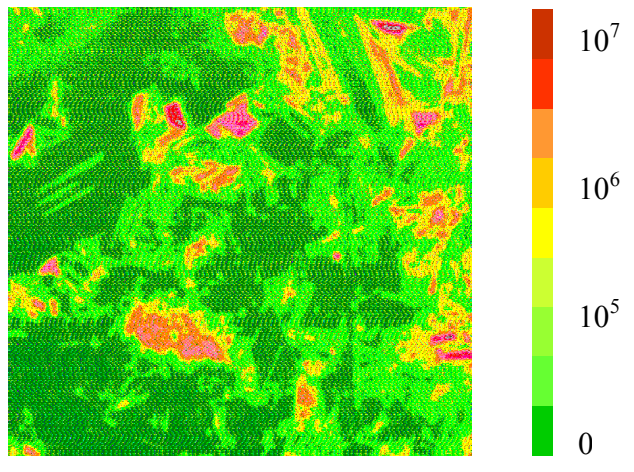

(a)

Figure 2. In (a), a defect map of a $5-\mathrm{cm}$ $x$ 5-cm section of a commercial mc-Si wafer. The scale is in defects $/ \mathrm{cm}^{2}$.

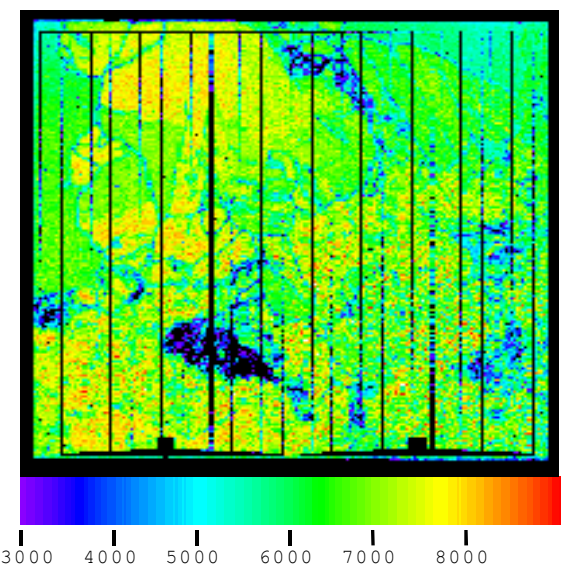

(b)

In (b), a photocurrent map of a solar cell fabricated on the wafer in (a), showing low response at defect clusters.

Impurities and defects present in the as-grown substrate strongly affect its material quality. In general, the minority-carrier diffusion length of as-grown substrates is low and varies spatially. The lowest minority carrier diffusion length occurs at defect clusters. Many approaches have been developed to mitigate the effects of defects and impurities. Impurity gettering is now extensively used in the silicon solar cell fabrication using phosphorous diffusion and Al alloying. Because these processes are generally combined with junction and contact formation processes, they need to be optimized for impurity gettering by maximizing the cell performance. Impurity gettering does not occur uniformly over the entire wafer - the regions with zero- or low-defect density exhibit 
very effective gettering while defect cluster regions do not getter well [10]. This effect can be seen from Fig. 2(b), which is photoresponse map of a solar cell fabricated on the wafer shown in Fig. 2(a). The map was generated by a long wavelength excitation that produces response proportional to the local minority-carrier diffusion length. The reason for a low gettering efficiency in defect cluster regions has been attributed to the presence of precipitated metallic impurities. Some initial calculations have shown that it would require many hours of annealing at high temperatures exceeding $1000{ }^{\circ} \mathrm{C}$ to dissolve such precipitates. The presence of impurity-precipitates poses another problem-if cell processing is done at temperatures near or exceeding $900{ }^{\circ} \mathrm{C}$, some of them dissociate to produce high concentrations of dissolved impurities which may not getter during the processing. In some cases this process can results in a degradation of the wafer quality [11].

Another approach to combat the influence of defects and impurities is to perform hydrogen passivation. Hydrogen passivation is very effective in some materials like edgedefined film growth and string ribbon silicon and may not produce significant effect on others. Recently, it has been possible to combine hydrogen passivation with PECVD nitride deposition used for antireflection coatings.

Theoretical analyses have shown that defect clusters limit the efficiency of many current commercial silicon solar cells. An interesting feature of defect clusters is that they primarily influence the voltage-related cell parameters without significantly lowering the photocurrent [9]. It is further shown that $18 \%$ efficiency cells can be fabricated if impurity precipitates within defect clusters can be gettered. This is a major effort within the DOE/NREL University Si Materials Research Program. It is important to recognize that formation of defect clusters is not inherent in the mc-Si growth, but is a result of thermal stresses associated with higher-growth speeds that result in formation of defects. However, at low thermal stresses, formation of defect clusters is preferred in mc-Si.

\section{INTERFACE AND PASSIVATION ISSUES FOR SCREEN-PRINTED CONTACTS}

One of the most difficult aspects of large-scale Si solar cell production is forming lowcost, high-quality front contacts. Screen-printing offers a simple, cost-effective method for contact formation that is consistent with the requirements for high throughput manufacturing $[12,13]$. The current problem with screen printing, however, is that the throughput gains are attained at the expense of device performance. The technical literature shows considerable scatter in fill factor values $(0.68-0.78)$ of screen-printed solar cells. In addition, there are no clear guidelines for achieving high fill factors reproducibly, as the problem requires a compromise among a variety of complex design issues. Therefore, an approach for understanding the critical interface metallization issues relevant to optimizing screen-printed metallization is required, recognizing the fact that fill factor can be degraded by gridline resistance, contact resistance, and contact formation induced junction leakage and shunting.

A combination of modeling, fabrication, characterization is necessary to provide guidelines for achieving high fill factors $(>0.78)$ on single crystal cells. The first step 
involves measuring metal resistivity as a function of firing temperature. For the $\mathrm{Ag}$ paste, metal resistivity decreases with the increase in firing temperature. In a recent study[13], for a firing time of $30 \mathrm{sec}$, the metal resistivity went below $3 \mu$-ohm-cm for firing temperatures above $700^{\circ} \mathrm{C}$. Model calculations indicated that $3 \mu$-ohm-cm is sufficient for grid design to achieve fill factor in excess of 0.78 . The next step involves measuring shunt resistance $\left(\mathrm{R}_{\mathrm{sh}}\right)$ as a function of firing temperature. In reference 13 , it was found that for a $30 \mathrm{sec}$ firing time, firing temperature should not exceed $730^{\circ} \mathrm{C}$ to maintain $R_{s h}$ in excess of $1 \mathrm{k} \Omega-\mathrm{cm}^{2}$, which is the second requirement for achieving fill factor in excess of 0.78 . The third step involves tailoring the junction depth for $730^{\circ} \mathrm{C} / 30$ minute firing cycle in order to minimize junction leakage current $\left(\mathrm{J}_{02}\right)$. It was found that $\sim 0.5 \mu \mathrm{m}$ deep junction with a sheet resistance of $\sim 40 \mathrm{ohms} / \mathrm{sq}$ was required for the above paste and firing condition to maintain $\mathrm{J}_{02}$ value below $10^{-8} \mathrm{~A} / \mathrm{cm}^{2}$, which is the third requirement for achieving $>0.78$ fill factor. The fourth step, a $400^{\circ} \mathrm{C} / 10 \mathrm{~min}$ forming gas anneal was found to be necessary for the above paste and firing conditions to reduce the series resistance to about $0.5 \mathrm{ohm}-\mathrm{cm}^{2}$. Systematic optimization of the firing cycle and junction depth, coupled with a post contact forming gas anneal, resulted in $17 \%$ efficient cells with fill factors in the range of $0.78-0.796$ on monocrystalline float-zone silicon. This approach is sensitive to paste composition, junction depth, substrate quality and firing equipment or cycle.

The processes required for high quality contacts to low-cost materials may be quite different due to a high defect density, defect density non-uniformity, and paste/defect interactions. In a recent study[13] conducted on mc-Si from Eurosolare S.R.L. corporation using rapid beltline processing of emitter and screen-printed contacts, a 40-45 $\mathrm{ohms} / \mathrm{sq}$ emitter was formed at $925^{\circ} \mathrm{C}$ in 6 minutes in a lamp heated beltline furnace. This resulted in a shallow-junction depth of $0.25 \mu \mathrm{m}$, which makes devices vulnerable to screen-printed, contact-induced junction shunting and leakage. In the course of investigating effects of paste composition on fill factor, it was found that impurities from the paste are able to get to the junction during a slow, prolonged firing cycle. It was found that rapid thermal processing during firing gave reasonable shunt and leakage current values but higher series resistance, preventing the fill factor from reaching 0.78 . The best mc-Si cell efficiency achieved in this study was $15 \%$, indicating the role of defects or paste/defect interaction in limiting the fill factor in $\mathrm{mc}-\mathrm{Si}$.

Since the fill factors achieved in these and other recent studies [14] are much greater than the fill factors $(0.68-0.75)$ of current industrial cells [15], there is a need for further development and technology transfer to bridge the gap between laboratory and industrial cells. Research should be conducted on fundamental understanding of fill-factor loss mechanisms associated with paste chemistry and composition, defect inhomogeniety and defect/paste interaction in order to achieve large area screen-printed cells reproducibly with high fill factor $(>0.78)$ on low-cost $\mathrm{Si}$ materials. These issues will become even more important for thinner silicon materials with more defects and smaller grain size. Development of a low-cost selective emitter, with $<40 \mathrm{ohms} / \mathrm{sq}$ diffusion underneath the grid and $>80 \mathrm{ohms} / \mathrm{sq}$ between the gridlines, may lead to significant improvements in the performance of screen-printed cells. Finally, fine-line printing, rapid thermal processing, 
and other novel low-cost contact formation techniques should be explored to reduce this dominant loss mechanism in the next generation silicon cells.

\section{THE BEHAVIOR OF HYDROGEN IN SILICON}

The understanding of the behavior of hydrogen in all forms of silicon continues to evolve and is an essential component of the science underlying silicon based photovoltaic technology. We describe here several interesting hydrogen-silicon phenomena that have emerged in the last few years that may provide research opportunities that are relevant to future silicon photovoltaics.

Hydrogen-silicon interactions have been found to be capable of cleaving macroscopic wafer-size thin $(<1 \mu \mathrm{m}$ thick $)$ layers of silicon from silicon crystals[16]. The hydrogeninduced cleaving process occurs through a series of steps in which hydrogen is implanted into silicon at high concentrations. A subsequent anneal initiates a planar cleaving process, resulting in a thin slab of silicon, either self supporting or transferable to other substrates[16]. The process has been employed extensively for the implementation of silicon integrated circuit fabrication in a silicon-on-insulator configuration, and hydrogeninduced cleaving processes for preparation of large $(200 \mathrm{~mm})$ silicon-on-insulator substrates are now entering high-volume manufacturing. A series of experimental findings have revealed a detailed picture of the process. Bech-Nielsen et al.[17] have considered the dilute limit, associated with isolated, hydrogen-coupled, point defects in silicon. The results identify a series of defect complexes, which can be described as $\mathrm{VH}_{\mathrm{n}}$, a vacancy with n-attached hydrogen atoms passivating the dangling bonds. Chabal and co-workers $[18,19]$ have used infrared spectroscopy to study the hydrogen-silicon system in the high concentration limit. The results illustrate the initiation of hydrogen decorated platelets which eventually initiate a cleaving process, driven by the trapped, highpressure gas. This process and the underlying science may play a significant role in further development of silicon-based photovoltaics employing thin crystalline silicon.

It has recently been discovered, and now well confirmed, that deuterium passivation of the $\mathrm{Si}-\mathrm{SiO}_{2}$ interface renders a metal-oxide-semiconductor field-effect transistor (MOSFET) very resistant to trap generation by hot electron impingement [20,21]. This has lead to speculation on the effect of deuterium passivation in other silicon-based materials and devices. Indeed, the stability of deuterated a-Si based solar cells [22,23] and deuterated terminated porous silicon [24] have been found to improve with the use of the isotope and have shown enhanced stability against degradation due to light and field exposure. The practical applicability of deuterium processing for all these systems is still under consideration. Nevertheless, it is clear that new understanding of the energetics and dynamics of hydrogen processes in silicon will emerge from this exciting research.

\section{THIN LAYER SILICON PHOTOVOLTAICS}

Although crystalline silicon technology, including both single crystal and multicrystalline, has been the dominant photovoltaics technology in the marketplace up to now, improvements in efficiency and reductions in cost/Watt for thin-film technologies (based, e.g., on amorphous silicon, cadmium telluride and copper indium diselenide) 
strongly suggest that a significant part of the future of photovoltaics will be defined by thin films. This has motivated the exploration of an analogous approach for silicon, called here "thin-layer" silicon technology in which the silicon absorber layer is not selfsupporting but is instead supported on a low-cost substrate (e.g., glass). A viable thinlayer silicon cell fabrication process, with its concomitant choice of low-cost substrate, must:

1. Yield module efficiencies of $\eta>13-14 \%$.

2. Demonstrate potential for lower cost/area $\left(<\$ 100 / \mathrm{m}^{2}\right)$ and/or cost/Watt $(<$ $\$ 2 / \mathrm{Wp}$ ) than competing "thick" c-Si, a-Si and compound thin-film technologies.

3. Have a fabrication throughput that can potentially equal or exceed process. throughputs for "thick" c-Si and a-Si.

4. Enable use of low-cost (e.g., glass or ceramic) substrates.

5. Have potential to reach large-scale production on a reasonable time scale (10 years or less).

A thin-layer silicon module with 13-14 \% efficiency will require: a sufficiently thick silicon layer $(\sim 5-30 \mu \mathrm{m})$ to achieve good red-spectral response, a thin-layer cell with base diffusion length in excess of the base thickness, adequate grain size $(\sim 10-60 \mu \mathrm{m})$, control of intragranular defects such as point defect complexes, dislocations and stacking faults, and light trapping on one or both sides of the active layers.

Several groups have investigated low temperature thin-layer polycrystalline silicon formation on glass for photovoltaic applications [25-29]. Polycrystalline silicon cells of $9.2 \%$ efficiency formed by solid phase crystallization were demonstrated several years ago [26]. Recently, a group at Kaneka Corporation demonstrated a completely stable, JQA-confirmed polycrystalline silicon cell of $10.7 \%$ efficiency in a $2.0 \mu \mathrm{m}$ thick silicon film grown by plasma-enhanced chemical vapor deposition (PECVD) on glass substrates [27]. These results establish the viability of thin crystalline silicon film materials for photovoltaics, and emphasize the extent to which careful attention to passivation and effective optical design in thin silicon cells can overcome the inherent disadvantage of crystalline silicon's indirect band gap.

To realize a practical thin-film silicon photovoltaics technology, several key problems and opportunities need to be addressed. For example, use of slightly thicker silicon films in the 5-20 $\mu \mathrm{m}$ range is expected to enable substantial improvements in cell short-circuit current, due to improved spectral response in the red and near infrared. However, growth of 5-20 $\mu \mathrm{m}$ silicon films is not currently practical due to low growth rates for the PECVD growth technique employed by Kaneka [3] and others. Kaneka reported a $7 \mu \mathrm{m}$ minoritycarrier diffusion length extracted from cell spectral response data, implying impressive defect passivation in their $2 \mu \mathrm{m}$ thick films with $\sim 1 \mu \mathrm{m}$ grain size. However, use of thicker silicon films in the 5-20 $\mu \mathrm{m}$ range will require substantially better quality material (with larger grain size and lower defect density) to enable minority-carrier diffusion lengths greater than or equal to the film thickness. Moreover, since material quality typically degrades with increasing growth rate in low-temperature vapor phase deposition 
processes, simultaneous achievement of high material quality and high growth rate for polycrystalline silicon is a significant challenge.
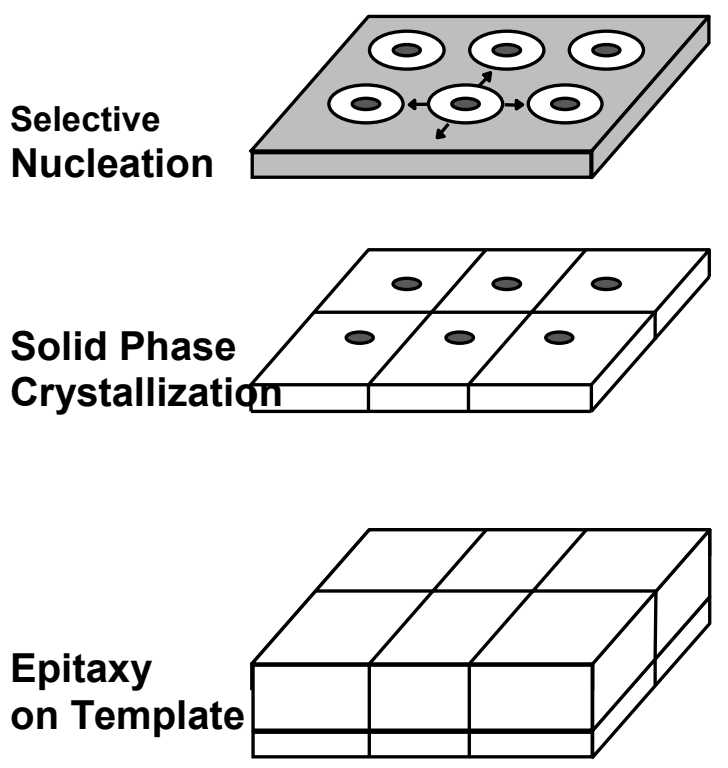

(a)

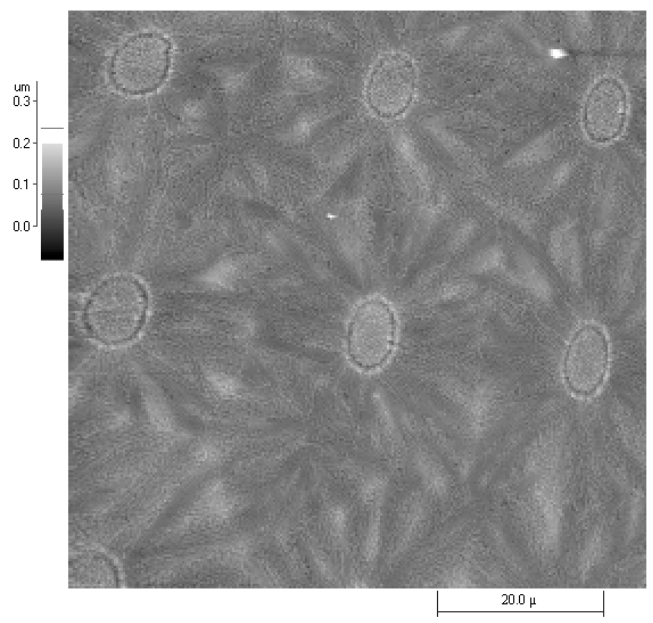

(b)

Figure 3. In (a), schematic of low-temperature process for formation of large-grained thin-layer silicon on low-cost substrates via selection nucleation and solid phase epitaxy[28]. Selective nucleation of crystalline silicon in an amorphous silicon film forms a large-grained template for a thicker epitaxial silicon layer. In (b), atomic force micrograph of $0.5 \mu \mathrm{m}$ crystal silicon grown at $T=490{ }^{\circ} \mathrm{C}$ on large-grained $(20 \mu \mathrm{m}$ grain size) Ge template formed at $T=400^{\circ} \mathrm{C}$.

Thus, development of polycrystalline silicon cell processes and cell efficiencies that are competitive with other thin-film technologies and "thick" crystalline silicon will require:

1. New approaches to improvement in thin-layer silicon material quality (increased grain size, lowered defect density) at the low temperatures demanded by use of lowcost substrates.

2. A breakthrough in thin-layer silicon growth rates, enabling polycrystalline silicon films of photovoltaically useful thickness to be grown while retaining high film quality.

3. A surface morphology with controlled roughness that enables enhanced optical absorption (i.e., "light-trapping"), preferably formed during growth.

Broadly, approaches to thin-layer silicon growth can be divided into two classes: lowtemperature processes $(\mathrm{T}<600 \mathrm{C})$ and high-temperature processes $(\mathrm{T}>900 \mathrm{C})$.

The biggest motivation for low-temperature processes is the existence of low-cost substrates available in large quantity (e.g., soda-lime glass) and the technology base related to the prior existence of another large-area, thin-film silicon electronics 
technology, namely, flat-panel displays. The challenges facing low-temperature, thinlayer silicon processes are many, because key steps in conventional crystalline silicon technology, such as crystal growth and junction formation, are done at temperatures of $\mathrm{T}$ $>900{ }^{\circ} \mathrm{C}$. The options for low-temperature crystal growth appear to be limited to solid phase crystallization, chemical vapor deposition or metal solution crystal growth. A large-grain silicon film made by a low-temperature process, selective nucleation and solid phase epitaxy (SNSPE)[28] is depicted in Fig. 3.

For high-temperature processes $(\mathrm{T}>900 \mathrm{C})$, which may potentially enjoy greater flexibility in cell process design than for low-temperature processes, the biggest challenge is to identify a demonstrably low-cost, useful substrate. The options for substrate formation may include speciality glasses and glass-ceramic materials, or sintered pressed ceramic sheets of SiAlON or related materials. A large-grain silicon film made by a high-temperature process, chemical vapor transport using the silicon tetraiodide reaction [29] is depicted in Fig. 4.

Figure 4. A $10 \mu \mathrm{m}$ thick silicon film grown at $\mathrm{T}=900{ }^{\circ} \mathrm{C}$ on high-

temperature glass substrates by chemical

vapor transport, yielding a grain size of 5-10 $\mu \mathrm{m}$ and an effective minority carrier lifetime of $5 \mu \mathrm{sec}$.

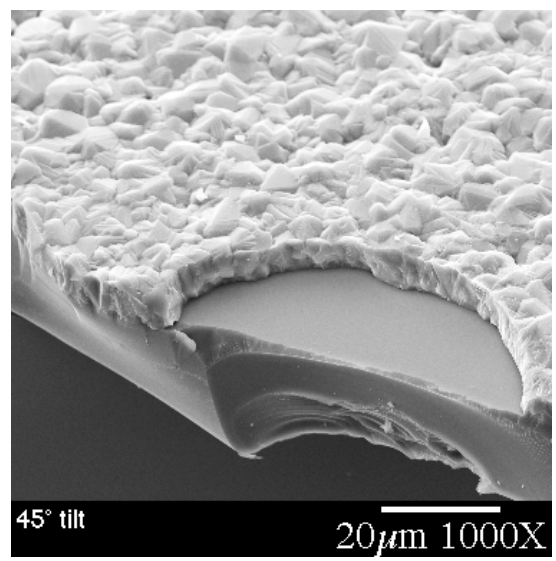

\section{CONCLUSIONS}

Crystalline silicon will remain as an important and possibly dominant technology in photovoltaics over the next 10-30 years, owing to its well-recognized desirable material properties and also to its established infrastructure for photovoltaic manufacturing. Basic research needs for the $21^{\text {st }}$ century include development of new separations processes for removing impurities from silicon feedstock, improved understanding of defect and impurity interactions in multicrystalline silicon, the development of novel, orientation-independent processes for light-trapping, surface passivation at contacts and other interfaces in thin Si structures, and improved understanding of the role of hydrogen in crystalline silicon.

Thin-layer silicon is now in the earliest stages of research, and the most important challenge at present is to grow a thin-layer silicon absorber on a low-cost substrate in a cost-effective manner that can achieve adequate photovoltaic performance. This will require new understanding of the kinetic limits to vapor phase $\mathrm{Si}$ deposition rates at low to intermediate temperatures, and understanding of the relation between vapor phase 
epitaxial crystal growth and defect generation. Other important issues for thin-layer silicon parallel those that are critical for thicker crystalline silicon. Achievement of good quality thin-layer silicon material on low-cost substrates will guide the way to progress in development of complete thin-layer silicon cell and module processes that can build upon experience gained from today's crystalline silicon photovoltaics technology.

\section{REFERENCES}

1. M.G. Mauk, P.E. Sims, and R.B. Hall, "Feedstock for Crystalline Silicon Solar Cells," American Institute of Physics Conf. Proc., 404, 21 (1997).

2. K.M. Mitchell, "The Status of Polysilicon Feedstock," American Institute of Physics Conf. Proc., 462, 362 (1998).

3. Summary of the Panel Discussions of the Sixth Workshop on the Role of Impurities and Defects in Silicon Device Processing, NREL/SP-413-21640, September 1996.

4. N. Nakamura, M. Abe, K. Hanazawa, H. Baba, N. Yuge, and Y. Kato, "Development of NEDO Melt-Purification Process for Solar Grade Silicon and Wafers," Proc. $2^{\text {nd }}$

World Conf. on Photovoltaic Solar Energy Conversion, 1193 (1998).

5. P. Menna, Y.S. Tsuo, M.M. Al-Jassim, S.E. Asher, R. Matson, and T.F. Ciszek, "Purification of Metallurgical-Grade Silicon by Porous-Silicon Etching," Proc. $2^{\text {nd }}$ World Conf. on Photovoltaic Solar Energy Conversion, 1232 (1998).

6. Y.S. Tsuo, J.M. Gee, P. Menna, D.S. Strebkov, A. Pinov, and V. Zadde, "Environmentally Benign Silicon Solar Cell Manufacturing," Proc. $2^{\text {nd }}$ World Conf. on Photovoltaic Solar Energy Conversion, 1199 (1998).

7. J. M. Gee, P. Ho, J. Van Den Avyle, and J. Stepanek, "Some Thermochemical Calculations on the Purification of Silicon Melts," $8^{\text {th }}$ Workshop on Crystalline Silicon Solar Cell Materials and Processes, August, 1998.

8. T.H. Wang and T.F. Ciszek, "Impurity segregation in LPE growth of silicon from $\mathrm{Cu}-$ Al solutions," Journal of Crystal Growth 174 (1997) 176-181.

9. B. L. Sopori, Proc. ICDS-19, Trans Tech Pub., Edited by G. Davies and M.H. Nazare, 527 (1997).

10. B. L. Sopori, L. Jastrzebski, T. Y. Tan, and S. Narayanan, Proc. $12^{\text {th }}$ PVSEC, 1003(1994).

11. B. L. Sopori, W. Chen, T.Y. Tan, and P. Plekhanov, National Center for Photovoltaics, Photovoltaics Program Review, AIP Conference Procd. 462, 341 (1998).

12. J. Nijs et. al., "Latest Efficiency Results with the Screen-printing Technology and Comparison with Buried Contact Structure," Proceedings of the $1^{\text {st }}$ World Conference on Photovoltaic Energy Conversion, Hawaii, 1242, (1994).

13. A. Rohatgi et. al., "Understanding and Implementation of Rapid Thermal Technologies for Low-Cost High Efficiency Silicon Solar Cells," to be published in IEEE Transaction on Electron Devices, (1999).

14. F. Duernickx et. al, "Simplified and Efficient Screen Printing Process for Multicrystalline Silicon Solar Cells Based on Firing Through Silicon Nitride," Proceedings of $14^{\text {th }}$ European Photovoltaic Solar Energy Conference, Barcelona, Spain, 792, (1997). 
15. T. Koval, J. Wohlgemuth, and B. Kinsey, "Dependence of Cell Performance on Wafer Thickness for BSF and non-BSF Cells, "Proceedings of $25^{\text {th }}$ IEEE Photovoltaic Specialists Conference, Washington, D.C., 505, (1996).

16. M. Bruel, Nucl. Instr. and Meth. B108, 313 (1996)

17. B.Bech Nielsen, L. Hoffmann and M. Budde, Matls Sci. and Eng. B36, 259 (1996).

18. M.K. Weldon, V. Marsico, Y.J.Chabal, S.B. Christman, E.E. Chaban, D.C. Jacobson, J.B. Sapjeta, A. Pinczuk, B.S. Dennis, A.P. Mills, C.A. Goodwin, and C.-M. Hsieh, Proc 1996 IEEE Int. SOI Conf., 150 (1996).

19. M.K. Weldon, Y.J. Chabal, D.R. Hamann, S. B. Christman, E.E. Chaban and L.C. Feldman, J. Vac. Sci. and Tech. B14, 3095, (1996).

20. J. W. Lyding, K.Hess, and I.C. Kizilyalli, Appl. Phys Let. 68, 2526 (1996).

21. K. Hess, I.C. Kizilyalli and J.W. Lyding, IEEE Trans. Elec. Dev., 45, 406 (1998).

22. J.H. Wei, M.S. Sun and S.C. Leee, Appl. Phys. Let., 71, 1498 (1997).

23. S. Sugiyama, J. Yang, and S. Guha, Appl. Phys. Let. 70, 378, (1997).

24. T. Matsumoto, Y. masumoto, T, Nakagawa, M. Hashimoto, K. Ueno, and N. Koshida, Jpn. J. Appl. Phys., 36, L1089 (1997).

25. See for example: K. Yamamoto, A. Nakajima, T. Suzuki, M. Yoshimi, H. Nishio and M. Izumina, Jpn.J. Appl. Phys. 33 L1751 (1994); R. Brendel, R. Bergmann, P. Lolgen, M. Wolf, and J. Werner, Appl. Phys. Lett. 70390 (1997); C.M Chen, C.M. Yang and H.A. Atwater, presented at $7^{\text {th }}$ NREL Workshop on Defects and Impurities in Silicon Device Processing, Vail CO, 1997; Z. Shi and S. Wenham, Prog. Photovoltaics, 2 153 (1994).

26. T. Matsuyama, N. Terada, T. Baba, T. Sawada, S. Tsuge, K. Wakisaka, and S. Tsuda, J. Non-cryst. Solids 200, 940 (1996).

27. K. Yamamoto, M. Yoshimi, T. Suzuki, Y. Tawada, T. Okamoto and A. Nakajima, presented at Spring 1998 Materials Research Society Meeting, San Francisco, CA; published in Proceedings of the EPVSEC, Barcelona, p. 1018 (1997).

28. "Polycrystalline Si Films Fabricated by Low Temperature Selective Nucleation and Solid Phase Epitaxy Process," C. M. Chen, and H. A. Atwater, in ThinFilm Structures for Photovoltaics, edited by E. D. Jones, J. Kalejs, R. Noufi, and B. L. Sopori, Mater. Res. Soc. Proc. 485, 67-72 (1998) .

29. T.F. Ciszek, private communication. 


\title{
AMORPHOUS AND MICROCRYSTALLINE SILICON SOLAR CELLS
}

\author{
Sigurd Wagner \\ Department of Electrical Engineering, Princeton University, Princeton, NJ 08544 \\ David E. Carlson \\ Solarex, 3601 Lagrange Parkway, Toano, VA 23168 \\ Howard M. Branz \\ National Renewable Energy Laboratory, Golden, CO 80401
}

\begin{abstract}
We review the progress made by amorphous silicon solar cells, including the emerging technology of solar cells of microcrystalline silicon. The long-term trend in the efficiency of stabilized laboratory cells based on a-Si:H has been a rise of $\sim 0.6 \%$ per year. The recent trend in the a-Si,Ge:H cell efficiency alone, measured in the spectral window assigned to the bottom device in a triple-junction cell, has been an increase of $\sim 0.16 \%$ per year. These improvements have brought within reach the target of $15 \%$ efficiency identified by EPRI and DOE for widespread application. Our review leads to an identification of areas of promising research, with emphasis on the fundamental science required to reach the $15 \%$ target, and then to move to the next-level efficiency goal.
\end{abstract}

\section{INTRODUCTION}

Solar cells of hydrogenated amorphous silicon and microcrystalline silicon are archetypal thin-film cells. They are thin, can be made in large area, and are made at low substrate temperatures. The low substrate temperatures of $\sim 150^{\circ} \mathrm{C}$ to $\sim 400^{\circ} \mathrm{C}$ provides flexibility in the choice of substrates, which includes plate glass and foils of steel or plastic. Hydrogenated amorphous silicon (a-Si:H) is finding growing use in other industries, which include activematrix, liquid-crystal displays (AMLCDs), electrophotography, application-specific sensor arrays on complementary metal-oxide semiconductor (CMOS) circuits, photosensor arrays for electronic cameras, and antifuses. This broadening range of applications multiplies the number of scientists and engineers that contribute to a-Si:H technology and provides increasing leverage to a-Si:H solar cell work. For example, equipment suppliers to the AMLCD industry are designing deposition systems for motherglass areas of $1-\mathrm{m}^{2}$ to $2-\mathrm{m}^{2}$ size, thereby helping solve questions of productivity, and of film uniformity over large area.

\section{The prospect for high efficiency.}

The present efficiencies of stable cells based on a-Si:H are [1]:

$\begin{array}{lc}\text { Single-junction a-Si:H } & 9.3 \% \\ \text { Triple-junction a-Si:H/a-Si,Ge:H/a-Si,Ge:H } & 13.0 \%\end{array}$


The microcrystalline cell has reached an efficiency of $8.5 \%$ [2]. Because predictive theories for the optical and electronic properties do not exist, cogent forecasts cannot be made of the efficiencies that are achievable with a-Si:H and $\mu \mathrm{c}-\mathrm{Si}: \mathrm{H}$. However, the steady efficiency increase of amorphous silicon cells documented in Figure 1 suggests that no plateau is in sight [3]. We proceed with a working assumption of physically achievable cell efficiencies in the neighborhood of $20 \%$ for single junctions and $30 \%$ for multijunctions.

\section{RESEARCH ISSUES OF THE PAST SEVEN YEARS}

In a previous workshop [4] seven groups of research issues with a-Si:H and its alloys were identified. The $\mu \mathrm{c}-\mathrm{Si}: \mathrm{H}$ cell was not yet known at the time of the 1992 workshop. The a-Si:H research issues were:

- Novel materials and growth methods

- Nanoscale structures and their effects on the electronic properties: structural and chemical heterogeneity

- Hydrogen configurations and their role in metastability

- Renewed studies of electronic transport

- Heterostructures and interfaces

- Impurities and defects

- Device modeling

\section{FUNDAMENTAL RESEARCH DONE IN THE PAST SEVEN YEARS}

Because of the large world-wide community in $\mathrm{R} \& \mathrm{D}$ on a-Si:H, an enumeration of all research accomplishments would go beyond the bounds of this report. Although the emphasis during this recent period lay on device work, very interesting fundamental results have been obtained and new techniques are coming to the fore. We first enumerate areas in which progress has been made, and then we illustrate the progress with a few arbitrarily chosen highlights.

Areas in which progress was made.

Novel materials and growth methods.

Protocrystalline silicon

Microcrystalline silicon

Hot-wire catalyzed growth

Nanoscale structures and their effects on the electronic properties:

structural and chemical heterogeneity.

SAXS

TEM microcrystalline inclusions

Hydrogen.

H-diffusion

H-recombination

Internal friction

Low $\mathrm{H}$ in hot wire 
Transport.

Hole transport

Alloys.

H dilution

Deposition.

High rate

Cluster formation

Impurities.

Correlation between impurity content and SW effect

Modeling.

AMPS established material property targets for stable 15\% triple-junction. AMPS modeling of device performance led to a revision of the mobility gap of a-Si:H and to a value for the discontinuity of the conduction-band edge between a-Si:H and $\mu \mathrm{c}-\mathrm{Si}: \mathrm{H}$.

The progress is easiest seen in illustrations that we provide in the following for some of the areas.

\section{Progress in cell efficiency.}

The steady rise in cell efficiency at an average rate of $0.6 \%$ per year has been maintained over the past seven years. Figure 1 [4] tracks the efficiency of a-Si:H based cell technology in the form of single-junction and multi-junction cells, and also shows the efficiency of modules. Note that the time delay between cell and module efficiency is only $\sim 5$ years, which reflects the fast technology transfer in the a-Si:H community. Recent progress in the efficiency of the low-gap, a-Si,Ge:H cell, used as the bottom device of triple-junctions, is shown in Figure 2 [5] The efficiency of Figure 2 is measured in the spectral window assigned to the bottom device in a triple-junction cell. It has been increasing at a rate of $\sim 0.16 \%$ per year. The efficiency improvements documented in Figures 1 and 2 have brought a-Si:H cell technology within reach of the present target of $15 \%$.

Hole mobility. The 1990s have seen widespread use and investigation of the techniques of hydrogen dilution [6] and of hot-wire catalyzed deposition [7, 8]. Their application has produced a-Si:H with hole mobilities in the 0.1 to $1 \mathrm{~cm}^{2} / \mathrm{Vs}$ range [9].

High-purity a-Si:H. Foreign impurities have been one suspected cause of metastable, lightinduced defects. In a series of experiments that included deposition of a-Si:H from highlypurified source gas in UHV-quality deposition systems, and secondary ion mass spectrometry (SIMS) at a high sputtering rate, the concentration of the atmospheric impurities of carbon, nitrogen, and oxygen in a-Si:H was brought to below the density of light-induced dangling bonds. This result rules out a one-to-one correspondence between light-induced defects and these impurities [10]. The concentration profiles of $\mathrm{O}, \mathrm{C}$ and $\mathrm{N}$ in the a-Si:H layer deposited on $\mathrm{x}-\mathrm{Si}$ is shown in Figure 3 [11]. The subgap optical absorption spectra before and after light-soaking are shown in Figure 4 [11]. 
Models of hydrogen in metastability. The role that hydrogen may play in the creation of metastable defects found ample attention during the period in review. One very interesting proposed mechanism of dangling-bond defect creation is by hydrogen collision [12]. In this model, shown in Figure 5, an incoming photon or an injected charge carrier releases a hydrogen atom from an $\mathrm{Si}-\mathrm{H}$ bond. The $\mathrm{H}$ atom diffuses through the network. If two diffusing hydrogen atoms open a weak $\mathrm{Si}-\mathrm{Si}$ bond and form two $\mathrm{Si}-\mathrm{H}$ bonds, the original sites that these two hydrogen atoms left behind remain dangling bonds. A new type of metastable defect associated with hydrogen is the H-flip defect [13]. The partial result of a molecular dynamics calculation of Figure 6 shows at the top the creation of the metastable $\mathrm{H}$ configuration as a result of a flip. The bars illustrate the change in electron density of several $\mathrm{H}$ atoms as a consequence of the flip. The flip changes the local structure around the $\mathrm{H}$ atom in question, but are not necessarily associated with the creation of a dangling bond.

Medium-range order, protocrystalline and microcrystalline silicon. Our view of the atomic arrangement in a-Si:H has become more refined in recent years, in part because of results obtained by new tools, and in part by the introduction of deposited microcrystalline silicon as a solar cell material. Small-angle X-ray scattering (SAXS) has shown that it is possible to make a-Si:H so free of micropores that the SAXS signal drops to the background level [14]. On the other hand, SAXS data from alloys clearly show microvoids, which may be preferentially oriented. Although voids have been drawing attention, crystalline inclusions also have come into focus. Under some growth conditions, such inclusions may be produced from clusters formed in the glow discharge $[15,16]$ Figure 7 illustrates the growth of negatively-charged clusters into particles that eventually become occluded in the growing film. A different path toward forming crystalline inclusions is taken when a-Si:H is grown under strong hydrogen dilution. Under these conditions, an initially pure a-Si:H film develops first protocrystallinity in the form of ordered regions and eventually microcrystalline inclusions. The transition from purely amorphous to microcrystalline silicon has been followed by ellipsometry, and characterized so extensively, that an a-Si:H - $\mu \mathrm{c}-\mathrm{Si}: \mathrm{H}$ has been established [17]. Figure 8 illustrates this phase diagram as a function of film thickness and hydrogen dilution, and shows that the transition depends on the type of substrate [18]. A new tool based on transmission-electron microscopy, fluctuation microscopy, is sensitive to variations in medium-range order and thus promises to provide information about the protocrystalline state [19]. The development of the microcrystalline silicon solar cell [2] showed that $\mu \mathrm{c}-\mathrm{Si}: \mathrm{H}$ is a semiconductor capable of bipolar operation, and has opened a new era in thin-film silicon technology. State-of-the-art cell performance is illustrated by Figure 9. [20]. 


\section{ISSUES THAT REMAIN RELEVANT}

Although the research emphasis in a-Si:H and $\mu \mathrm{c}-\mathrm{Si}: \mathrm{H}$ has varied over the years, most issues remain active, if only because the rising demands on cell performance require ever deeper understanding. Solar cells are high-performance analog devices, with highly interrelated parameters. Therefore, it is not surprising to see that solving one issue exposes another issue that must be solved. All issues in a-Si:H and $\mu \mathrm{c}-\mathrm{Si}: \mathrm{H}$ are connected. We seek to illustrate this connection with the following table.

Hierarchy and Connection of Issues:

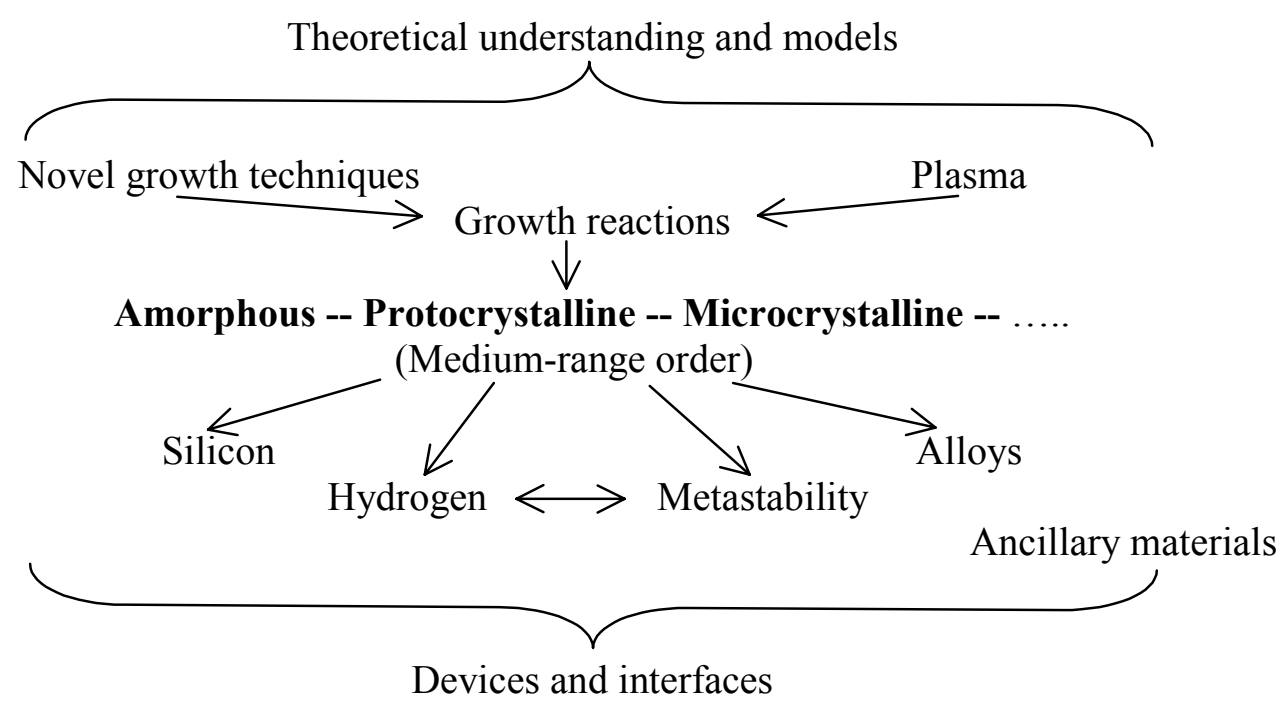

\section{Discussion of issues.}

In this section, we list input collected from our colleagues in the a-Si:H and $\mu \mathrm{c}-\mathrm{Si}: \mathrm{H}$ research community. The input is grouped by entries in the table above.

1. Amorphous -- Protocrystalline -- Microcrystalline -- .

a-Si is the end point of a continuum

Is the in-between (protocrystalline, on-the-edge) material really different?

Develop quantifiable measures for this difference

Difficulty of controlling large-area uniformity of protocrystalline silicon

Make the ideal material: absorption of a-Si, transport of $\mathrm{x}-\mathrm{Si}$

Understand and control the a/c transition

Nanocrystalline Si: electron states, doping, recombination, and confinement effects?

Relation between crystallinity and optical-absorption coefficient

Grain boundary properties, including those of $\mathrm{Si}, \mathrm{Ge}$ and $\mathrm{Si}, \mathrm{C}$ alloys

Need methods for characterizing electronic and optical properties of mixed-phase materials, and materials with continuously varying structure

Need more and cleverer structural probes

Transport properties: compare materials for high hole mobility 
What are the commonalties in structure that provide high mobility (substrate, growth)?

Growth parameters that affect medium-range order, protocrystallinity and microcrystallinity:

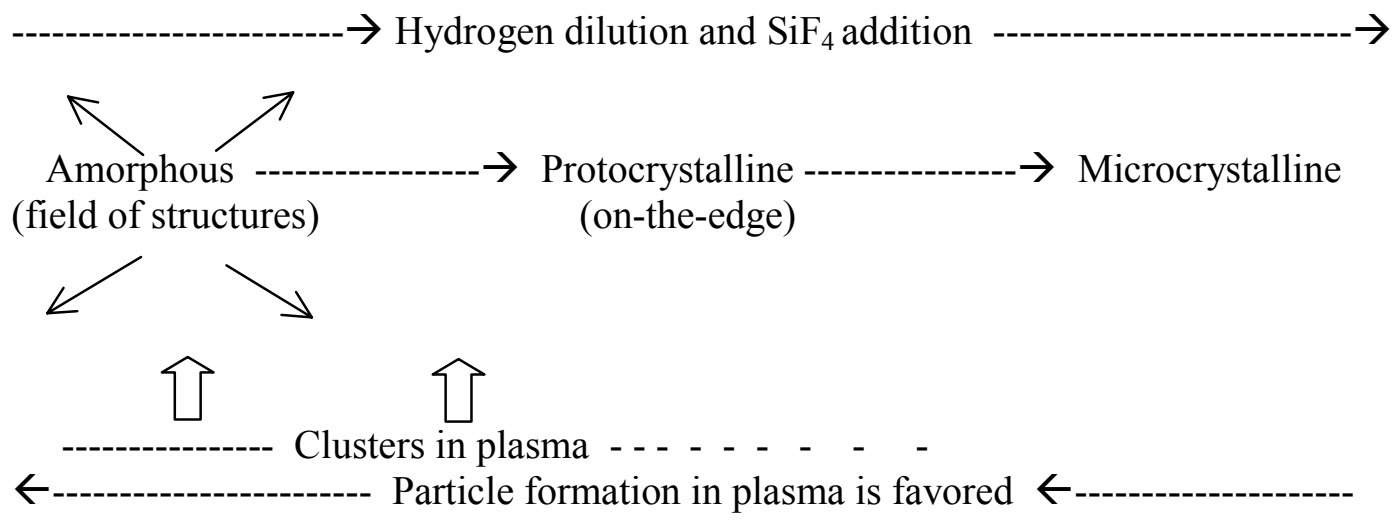

2. Medium-range order.

Which difference between PECVD a-Si:H, H-diluted a-Si:H, hot-wire a-Si:H

Techniques for measuring MRO

3. Theoretical understanding and models.

Medium-range order, dangling bonds, amorphous/crystalline interfaces, transport, recombination in materials

Need first-principles calculation of electronic structure

More extensive use of molecular dynamics computation

Model growth: gas phase and surface

Lack of critical mass in theory

Growth, electronic, device, optical models

4. Metastability.

Theory of light induced degradation

Does a single event produce dangling bonds and structural changes?

Do structural changes precede the creation of dangling bonds? Which precedes what?

Time-resolved measurements

Sources of irreversibility

Irreversibility arising from trace amounts of boron

5. Hydrogen.

How does $\mathrm{H}$ affect the network, how does the network affect $\mathrm{H}$ ?

Structure/configuration and topology

$\mathrm{H}$ microstructure other than by (gross) IR absorption

$\mathrm{H}$ models for a-Si:H with inhomogeneous structure

Describe how $\mathrm{H}$ breaks bonds as it moves and leaves structural changes in its wake

Localized H motion 
Effect of charge state on $\mathrm{H}$ motion

Relation to metastability of $\mathrm{H}$ structures

$\mathrm{H}$ vs. D: changes discharge kinetics, not film itself? Make the same films with H and D to test

Effect of other bond terminators, $\mathrm{F}, \mathrm{Cl}$ on discharge and in film

6. Plasma.

Physics and chemistry of plasma processes, dependence on excitation frequency

Transients in plasma deposition

High deposition rate (importance for capital cost), indispensable for practical use of $\mu \mathrm{c}-\mathrm{Si}$

What to do to keep particles out (a-Si:H) or to include them (protocrystalline $\mathrm{Si}$ )

Homogeneity over large area. Electrode spacing, showerhead structure, excitation

frequency

The following schematic diagram [16] illustrates the relation between precursors and particle growth:

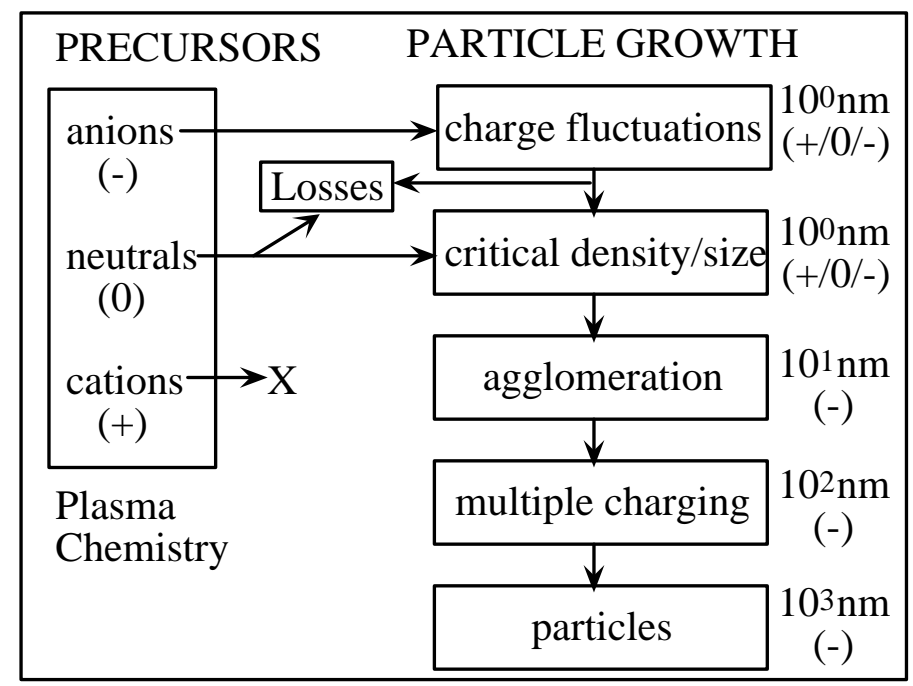

7. Novel materials and growth techniques.

Create materials with designed optical absorption and transport properties. Can we make a material with the optical-absorption characteristics of a-Si:H (Figure 10) [20] and the transport properties of $\mathrm{x}-\mathrm{Si}$ ?

Designed mixtures of amorphous and crystalline, design quantum properties

Low-pressure, plasma-free techniques: hot wire, ...

Hot wire: filament stability and life, chamber-induced differences

Design remote reactors to separately control feed of reactive species and of particles, and of growth reactions on the surface

Develop techniques for designing clean reactors

8. Growth reactions.

Systematic study of the chemistry of growth reactions

Relation between growth chemistry - structure- electro-optic properties - device performance 
Material and device must be made in the same system

Multiple-zone reactors for the manipulation of film properties (e.g., a small density of nuclei is established in zone 1, and is injected as seed into surface in zone 2 to control growth)

Reactions in hot wire deposition

Induction period for microcrystalline growth

Raise growth rate of microcrystalline silicon

Microcrystalline Si for tunnel junctions is the least controlled industrial material at present

Effect of substrate on film structure (High- $\mu_{\mathrm{p}}$ material grows best on $\mathrm{x}-\mathrm{Si}$. Hot wire produces epitaxial $\mathrm{Si}$ at $300^{\circ} \mathrm{C}$ if surface is cleaned properly.)

Growth chemistry of alloys

9. Alloys.

Bring understanding and control of a-Si,C:H and a-Si,Ge:H to the level of a-Si:H

What did improve in a-Si,Ge:H when USSC raised a-Si,Ge:H cell efficiency?

Different role of $\mathrm{H}$ in different alloys: clustered on $\mathrm{C}$ in a-Si, $\mathrm{C}: \mathrm{H}$, clustered on $\mathrm{Si}$ in a-

$\mathrm{Si}, \mathrm{Ge}: \mathrm{H}$

Understand doping of alloys

To date mostly alloys with group IV partners: explore others, Si-Se, Si-metals

Precursor molecules for alloys

Very little basic work now

10. Devices and interfaces.

Fundamentals of devices: assemblies of thin films of varying structure, interfaces

Superlattice structures for the study of interfaces

Limits to $\mathrm{V}_{\mathrm{oc}}$ : band tails, interfaces, band alignments

Devices including a range of structures: amorphous in-between, microcrystalline

Measure device physics by other than solar cell parameters (e.g., EL, capacitance, transient transport)

Interfaces: measure, describe, and model

Understand role of buffer layers. Why are they needed?

Connection between device performance and material quality: how strong is it?

11. Ancillary materials.

Transparent conductors

Formulation of optical waveguiding

Minimizing materials use with thin materials

Flexible substrates

Mechanical properties, stress

12. Combinatorial techniques for speeding up research.

NEW FUNDAMENTAL RESEARCH OPPORTUNITIES

The preceding list of research issues already implies and reflects a host of research opportunities. With the following list we take a step back and survey the broader areas of 
research that we consider important to continued progress in a-Si:H and $\mu \mathrm{c}-\mathrm{Si}: \mathrm{H}$ solar cell technology.

Experiment with new growth techniques

Gas-phase chemistry of glow discharge and hot wire

Surface reactions, nucleation and growth, substrate effects

Molecular dynamics of large cells: $10^{8}$ atoms

Techniques to measure and interpret medium-range order

Protocrystalline material: how does it differ from a-Si:H

Nano-, microcrystalline material: induction period, substrate effect, optical and electronic properties, quantify grain boundaries, doping

Design silicon with a high optical-absorption coefficient and long transport length

Early kinetics of metastability: sequence of changes in structure and defects

Probes for hydrogen topography

Role of alternative bond terminators: $\mathrm{F}, \mathrm{Cl}$

Alloys: what makes a-Si,Ge:H and a-Si,C different from a-Si:H

Doping of alloys

Make and measure microcrystalline alloys

Make alloys with non-column IV elements

Establish complete models: Growth, structure, electrical, optical, including interfaces

\section{COMMONALITIES WITHIN PHOTOVOLTAICS}

Amorphous -- Protocrystalline -- Microcrystalline -- ....., Hydrogen

Relation to single-, polysilicon

Plasma, Novel growth techniques, Growth reactions

Plasma processing, surface reactions

Devices and interfaces

Numerical modeling for all thin-film cells: Optical, electronic, Ancillary materials: TCOs

\section{OUTLOOK}

We consider the following three directions of basic research crucial to continued progress in a-Si:H and $\mu \mathrm{c}-\mathrm{Si}: \mathrm{H}$ solar cell technology.

1. The understanding and control of Si and alloy film structure, ranging from amorphous over MRO and protocrystalline to $\mu \mathrm{c}$ material, with increasing emphasis on high deposition rate as the structural order increases.

2. The acquisition of a comprehensive understanding of the role of $\mathrm{H}$ in establishing nanostructure, in alloying and doping, in metastability, and as a structural modifier during solar cell operation.

3. The understanding and control of the gas-phase chemistry, the reactions on the growing surface of Si and alloy films, and their effect on device properties. 


\section{ACKNOWLEDGEMENTS}

We gratefully acknowledge receiving figures and comments from our colleagues R. Biswas, R.W. Collins, J.M. Gibson, S. Guha, Ch. Hollenstein, A. Howling, T. Kamei, H. Meier, E.A. Schiff, A. Shah, and P. Voyles, and advice from J.R. Abelson, R. Crandall, V.L. Dalal, S.J. Fonash, H. Fritzsche, A.C. Gallagher, G. Ganguly, R. Gordon, D. Han, S. Hegedus, T.M. Peterson, R.A. Street, D.L. Williamson and K. Zweibel. Without their swift response we would not have been able to pull this information together on short notice.

\section{REFERENCES}

(1) S. Guha and J. Yang, Record of the Eleventh NREL-EPRI Amorphous Silicon Guidance Team Review Meeting, April 9-10, San Francisco. NREL, Golden, CO 1999. J. Yang, A. Banerjee, and S. Guha, Appl. Phys. Lett. 70, 2975 (1997)

(2) J. Meier, R. Flückiger, H. Keppner, and A. Shah, Appl. Phys. Lett. 65, 860 (1994). J. Meier and A. Shah, Université de Neuchâtel, Switzerland, private communication 1999

(3) B. Stafford and B. von Roedern, NREL, private communication, 1999

(4) W.A. Paul, R.A. Street and S. Wagner, in J. Electronic Materials 22, 39 (1993)

(5) S.Guha, United Solar Systems Corporation, private communication, 1999

(6) S. Guha, K.L. Narasimhan and S.M. Pietruszko, J. Appl. Phys. 52, 859 (1981)

(7) H. Matsumura, Mat. Res. Soc. Symp. Proc. 557, to be published

(8) A.H. Mahan, J. Carapella, B.P. Nelson, R.S. Crandall and I. Balberg, J. Appl. Phys. 69, 6728 (1991)

(9) Ganguly et al., Jpn. J. Appl. Phys. 34, L227, 1995. E.A. Schiff, Syracuse University, personal communication, 1999

(10) T. Kamei, N. Hata, A. Matsuda, T. Uchiyama, S. Amano, K. Tsukamoto, Y. Yoshioka, and T. Hirao, Appl. Phys. Lett. 68, 2380 (1996). T. Kamei et al., J. Vac. Sci. Tech. A, 17, 113 (1999)

(11) T. Kamei, personal communication 1999

(12) H. Branz, Phys. Rev. B 59, 5498 (1999)

(13) R. Biswas, private communication 1999

(14) D.L. Williamson, Mat. Res. Soc. Symp. Proc. 377, 251 (1995)

(15) A. Gallagher, Mat. Res. Soc. Symp. Proc. 557, to be published

(16) J. Perrin and Ch. Hollenstein, "Dusty Plasmas Between Science and

Technology," ed. A. Bouchole, Wiley, New York, 1999, in press. A.A. Howling, C. Courteille, J.-L. Dorier, L. Sansonnens and Ch. Hollenstein, Pure and Appl. Chem. 68, 1017 (1996).

(17) J. Koh, Y. Lee, H. Fujiwara, C.R. Wronski, and R.W. Collins, Appl. Phys. Lett. 73, 1526 (1998).

(18) R.W. Collins, private communication 1999.

(19) J.M. Gibson and M.M.J. Treacy, Phys. Rev. Lett. 78, 1074 (1997); J.M. Gibson,

M.M.J. Treacy and P. Voyles, Appl. Phys. Lett. 73, 3093 (1998)

(20) H. Meier and A. Shah, private communication 1999 


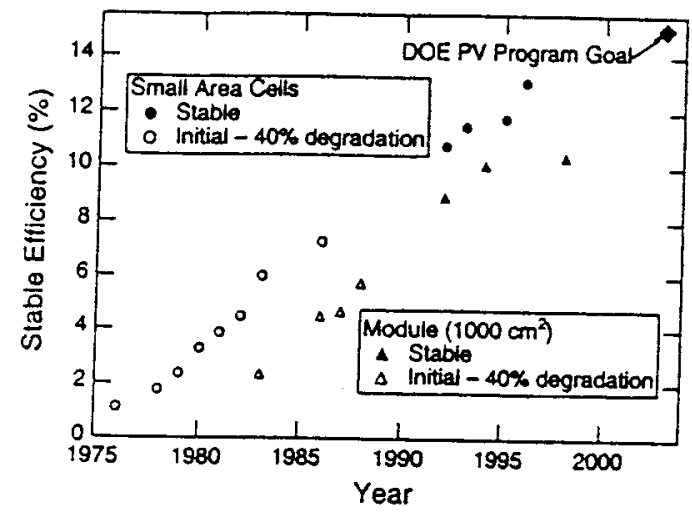

Figure 1. Chronological record of the efficiencies of a-Si:H based solar cells and modules (Ref 3 ).

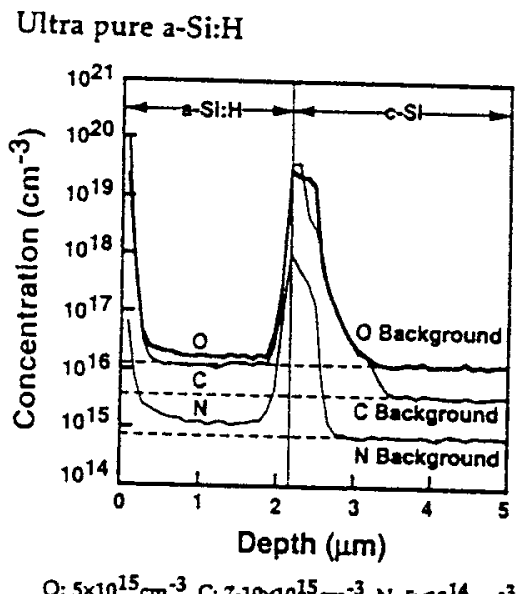

Very high primary ion beam current density high rate sputtering by primary ions

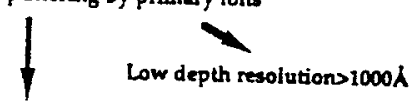

High Sensitivity

(Nornal detection libnit of STMS : $10^{17} \mathrm{~cm}^{-3}$ for $\mathrm{O}$ or C. $10^{16} \mathrm{~cm}^{-3}$ for $\mathrm{N}$ )

Figure 3. SMS profile of an ultra-high purity sample of a-Si:H, made to correlate impurity content and light-induced defect generation (Ref. 10).
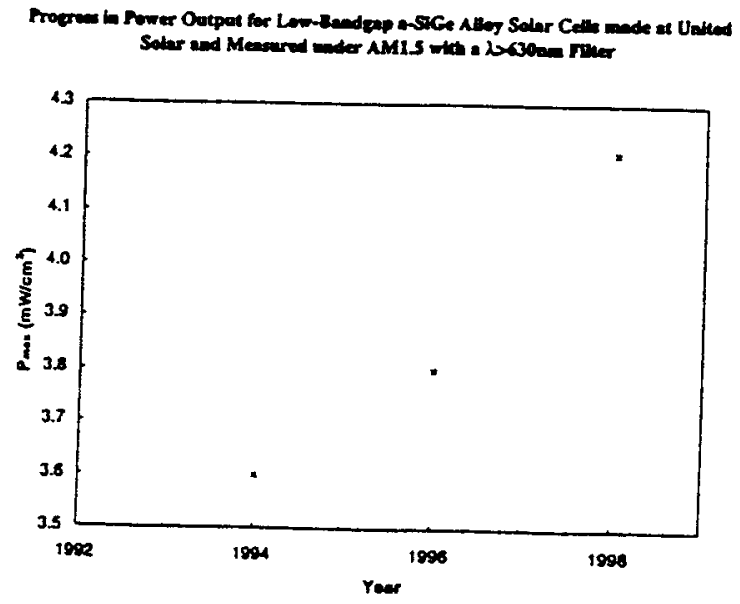

Figure 2. Chronological record of the efficiency of the low-gap a-Si.Ge:H cell for use in a triple junction devices.

Efficiency measured under filtered red light (Ref. 5).

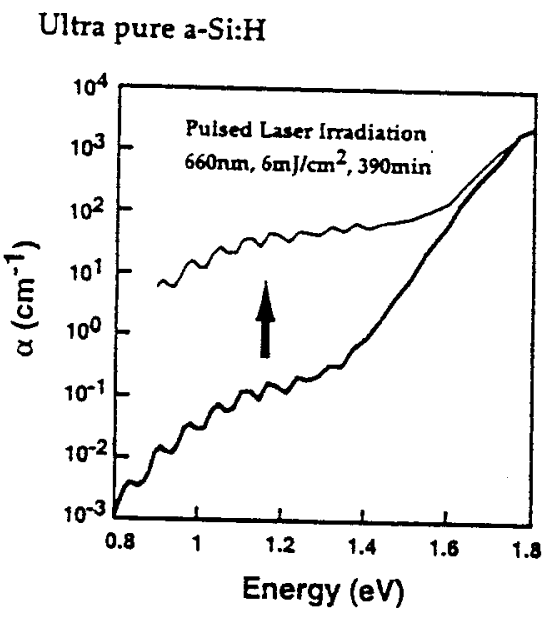

LID density >> Impurity contents Extrinsic model which postulates
one-to-one correlation between LID and impurity

Figure 4. Subgap optical absorption of an ultra-high purity sample of a-Si:H, which exhibits a saturated density of defects higher than the concentration of atmospheric impurities (Ref. 10). 


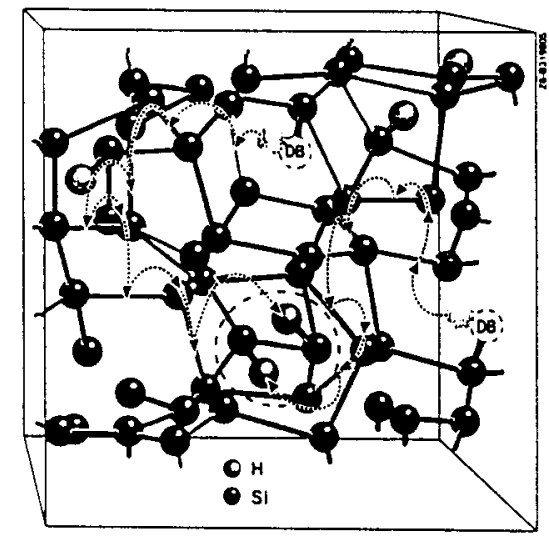

Figure 5. Illustration of the hydrogen collision model for the generation of metastable dangling bonds in a-Si:H (Ref. 12).

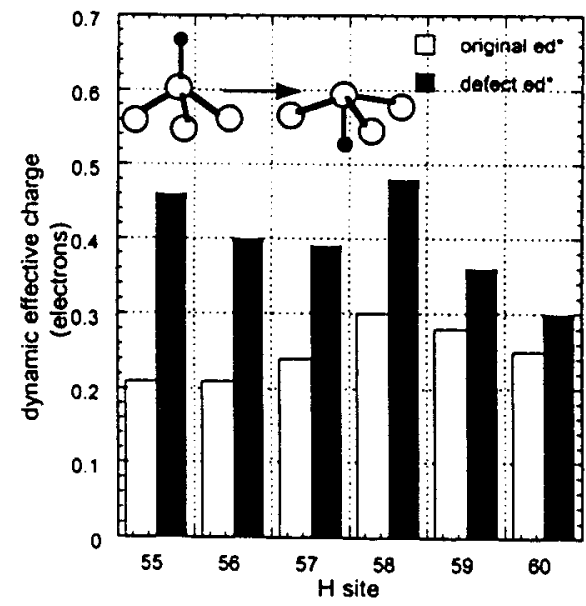

Figure 6. Mlustration of the hydrogen flip metastability, and of the effective electron charge on a set of hydrogen atoms before and after the flip process (Ref. 13).
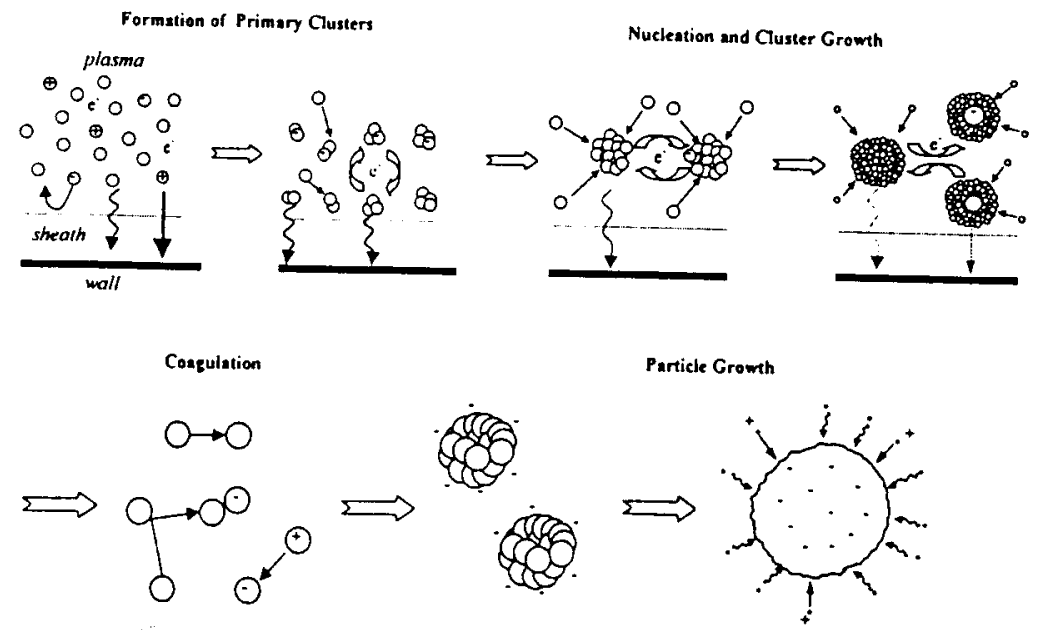

Figure 7. Formation of particles in a silane glow discharge in four steps: cluster formation, particle nucleation, coagulation, and independent growth of trapped multiplycharged macroscopic particles by condensation. (Ref. 16). 


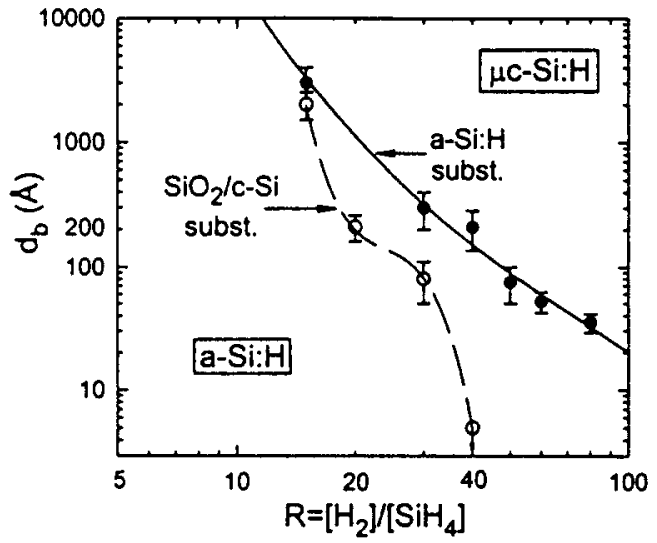

Figure 8. "Phase diagram" of a-Si:H and $\mu \mathrm{c}-\mathrm{Si}: \mathrm{H}$ as a function of hydrogen dilution and film thickness. Not that the "phase boundary" depends on the type of substrate on which the film is grown (Refs. 17 and 18).

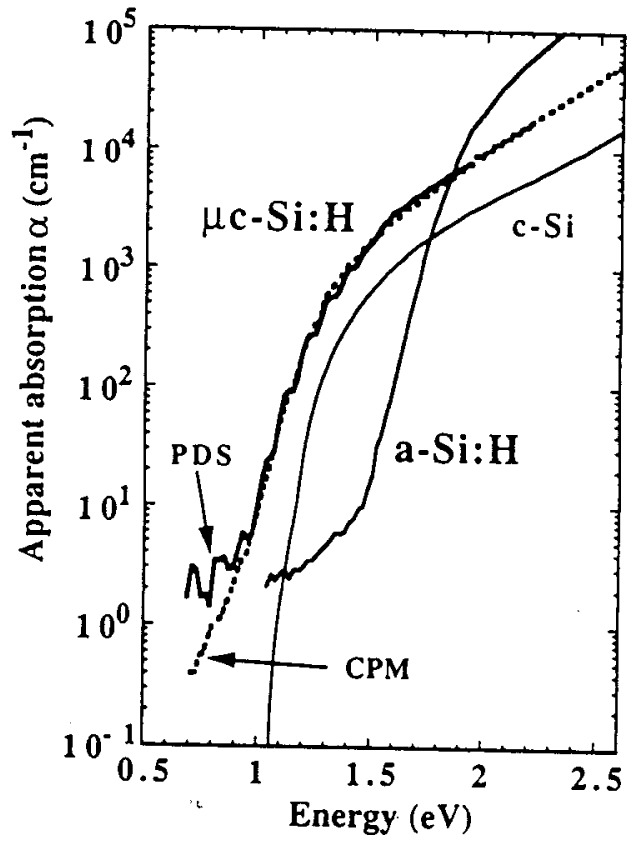

Figure 10. Optical absorption spectra of $\mu c-S i: H, a-S i: H$ and $x-S i$ (Ref. 20).

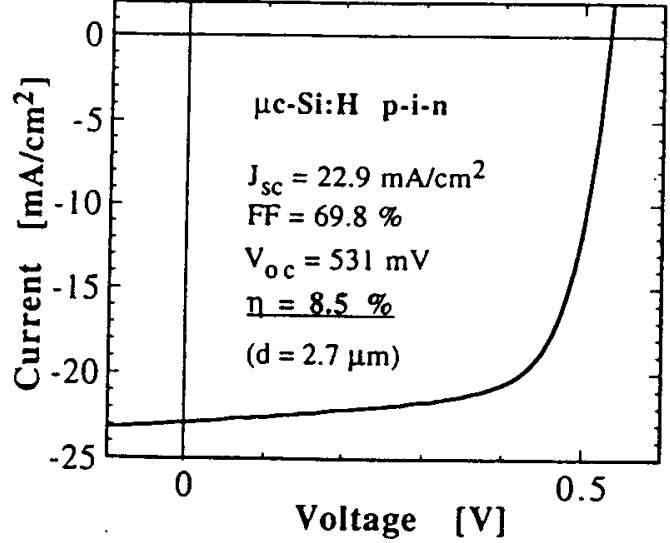

\begin{tabular}{|c|l|l|l|l|l|l|l|}
\hline$\eta[\%]$ & 7.7 & 8.3 & 7.7 & 8.1 & 8.5 & 3.2 & 4.4 \\
\hline $\mathrm{J}_{\mathrm{sc}}\left[\mathrm{mA} / \mathrm{cm}^{2}\right]$ & 25.3 & 25.2 & 21.5 & 23.2 & 22.9 & 18.4 & 17.9 \\
\hline FF $[\%]$ & 67.9 & 68.2 & 71.1 & 68 & 69.8 & 30.5 & 41.8 \\
\hline$V_{\text {oc }}[\mathrm{mV}]$ & 448 & 483 & 503 & 512 & 531 & 568 & 592 \\
\hline
\end{tabular}

Figure 9. Current-voltage characteristic of a state-of-the-art solar cell made with a $\mu \mathrm{c}-\mathrm{Si}: \mathrm{H}$ absorber layer. The table lists the characteristics of several such cells and highlights the ability to exceed a $\mathrm{V}_{\mathrm{oc}}$ of $0.5 \mathrm{~V}$ (Ref. 20). 


\title{
BASIC RESEARCH OPPORTUNITIES IN CU-CHALCOPYRITE PHOTOVOLTAICS
}

\author{
A. Rockett, ${ }^{(a)}$ R.N. Bhattacharya,${ }^{(b)}$ C. Eberspacher, ${ }^{(c)}$ V. Kapur, ${ }^{(d)}$ and S.-H. Wei ${ }^{(b)}$
}

(a) University of Illinois, 1-107 ESB, MC-233, 1101 W. Springfield Ave., Urbana, IL 61801

(b) National Renewable Energy Laboratory, 1617 Cole Boulevard, Golden, CO 80401-3393

(c) UNISUN, 587-F North Ventu Park Road, PMB 124, Newbury Park, CA 91320-2723

(d) ISET, 8635 Aviation Boulevard, Unit B, Inglewood, CA 90301

\begin{abstract}
A brief review is presented of fundamental research topics of primary importance to the development of improved solar cells based on chalcopyrite-structure materials. The opinions presented are a consensus opinion of the authors of the paper, with input from members of the chalcopyrite solar cells research community in the United States. Major topical areas identified included, in order of importance, are 1) development of an integrated predictive understanding of CIGS(S) materials and devices, 2) development of novel deposition techniques and characterization of the mechanisms of growth in existing and novel processes, 3) novel materials, especially with wide-energy gaps $(\geq 1.7 \mathrm{eV})$ other than $\mathrm{Cu}$-based chalcopyrites, 4) development of real-time material characterizations for process control, and 5) alternative front- and rearcontact materials. Although the five topics identified are quite broad, they do not include all topics of interest. Also discussed briefly are some other potential research areas not in the highest priority topics, in particular, areas identified as primarily "engineering" rather than "science."
\end{abstract}

\section{INTRODUCTION}

This paper presents the results of a discussion of basic science research topics of primary importance in developing improved solar cells based on chalcopyrite-structure materials. Participants in the discussion were the authors of this paper, with input from a number of other experts in the field. The discussion was held May 3, 1999, in Seattle, Washington, as part of the Basic Research Opportunities in PV Workshop.

\section{I.A. Significance of Chalcopyrite-Structure Solar Cells}

Solar cells based on chalcopyrite materials are produced from thin-film layers on inexpensive soda-lime glass substrates. The specific materials used in the best devices are $\mathrm{Cu}\left(\mathrm{In}_{1-\mathrm{x}} \mathrm{Ga}_{\mathrm{x}}\right) \mathrm{Se}$ [CIGS] alloys, although other highly efficient devices have been produced with some selenium replaced with sulfur [CIGSS] and with pure $\mathrm{CuInSe}_{2}$ (CIS). Significant advantages of these alloys include: they span the energy-gap range needed to make the highest efficiency single- and 
multijunction solar cells, they work well in the form of small-grained $(\sim 1 \mu \mathrm{m})$ polycrystals, and they are not strongly sensitive to impurities and crystalline defects. It is important that the materials function well in thin-film form, as thin-film manufacturing processes are well known to be scalable and capable of coating very large areas of glass at practical costs. It will be necessary to produce thousands of square kilometers of solar cells to make a major contribution to world energy needs.

CIGS(S) $\left[\mathrm{Cu}\left(\operatorname{In}_{1-\mathrm{x}} \mathrm{Ga}_{\mathrm{x}}\right) \mathrm{Se}_{2}\right.$ or $\left.\mathrm{Cu}\left(\operatorname{In}_{1-\mathrm{x}} \mathrm{Ga}_{\mathrm{x}}\right)\left(\mathrm{Se}_{1-\mathrm{y}} \mathrm{S}_{\mathrm{y}}\right)_{2}\right]$-based solar cells have the highest verified efficiencies in both small-area devices and large-area $\left(4000 \mathrm{~cm}^{2}\right)$ modules, $18.8 \%$ and $12.1 \%$, respectively, of any thin-film photovoltaic technology. Performances exceeding $16 \%$ conversion efficiency are achieved routinely in a number of laboratories worldwide. Test modules and arrays based on CIGS(S) alloys have been operating outdoors for over 10 years with little degradation. Furthermore, no increase in degradation has been found as overall performance of devices and modules has improved. The prospects for CIGS(S) solar cell technology changed significantly in the past year when Siemens Solar Industries began selling modules based on CIGS(S). Several other manufacturers are making exciting progress, and they appear poised to enter the market with products of their own in the future.

Not only do CIGS(S) solar cells hold the current thin-film performance records, but these records have also been achieved in single-junction devices with energy gaps that are not optimized to the solar spectrum. Significant further advances can be anticipated with either tandem-structure devices or with larger energy gaps in the absorber layers. By contrast, competing technologies have either nearly fully exploited the potential of multijunction devices (as in the case of amorphous $\mathrm{Si}$ ) or lack the broad range of alloys needed to make a multijunction device (as for CdTe).

In spite of the many advantages of CIGS(S), major hurdles exist before it can achieve its optimal performance and lowest cost of manufacturing. The most significant hurdle is the lack of a sufficient understanding of the materials, processing methods, and devices. A number of senior members of the CIGS(S) community producing high-performance devices have been heard to make comments that can be paraphrased as, "We do it, but we do not fully understand why it works." Optimization of current devices and the planning and design of new devices are often done empirically, based on experience with what worked before or based on "conventional wisdom." As the scientific understanding of CIGS(S) materials has gradually progressed, many of these assumptions have been proven wrong or misguided. Unfortunately, most major questions remain unsatisfactorily understood, or serious disagreements exist about the validity of the conclusions. Consequently, the research topics needing immediate attention include some relatively elementary questions.

These problems are magnified by the fact that there is hardly any research on chalcopyrites outside the photovoltaics (PV) community. Thus, while the PV community working on Si or IIIVs can benefit from the tremendous investment that has existed outside PV in basic materials science of Si and III-Vs, the PV community working on CIGS(S) has to carry out its own materials science research.

In addition to basic science issues directly addressing the problems of current-generation CIGS(S) devices, future-generation devices require major scientific research. Questions can be divided into a clearer set addressing specific needs of next-generation devices that will evolve predictably from current devices, and more speculative questions such as whether some undiscovered material exists that can radically improve all devices. In other words, both evolutionary and revolutionary scientific approaches to next-generation technologies are worthy of effort. The 
recommendations presented here include research addressing both current and future-generation devices through both evolutionary and revolutionary pathways.

In summary, the potential benefits to basic science research in CIGS(S) materials, processes, and device physics are large, and the problems needing attention are fairly clear.

\section{I.B. Objectives of Basic Science for Chalcopyrite Devices}

When laying out recommended areas of research, it is important to state in advance the objectives of that research. We considered the time scale of application of any potential research recommended and concluded that dramatic benefits can be expected on all time frames. However, such significant benefits can be anticipated from research in support of current technologies that the highest-priority recommendations address these topics. The results will also be fundamental to planning future generations of devices and even to design of cost-effective and well-organized scientific research exploring the possibility of future device designs. We believe that scientific research on CIGS(S) will yield conclusions that will reach beyond solar cells. With this in mind, we suggest that the scientific objectives of a basic research program in CIGS(S) should be as follows.

- To conduct fundamental experiments leading to broadly applicable physical and chemical principles specifically addressing the material properties and processing of CIGS(S).

- To develop from these results novel materials and processes and quantitative and predictive models of materials and devices.

- To develop the science required for methodical design and manufacture of next-generation chalcopyrite-based solar cells.

- To explore potential revolutionary advances in chalcopyrite materials and processes.

\section{I.C. Current Understanding of Chalcopyrite Materials and Solar Cells}

Recent experiments have yielded extremely encouraging results in small and module-sized devices based on CIGS(S) alloys. The best devices generally have alloys containing a majority of CuInSe $e_{2}$, with additions of $\mathrm{CuGaSe}_{2}$ and $\mathrm{CuInS}_{2}$ at the back and front surfaces in some devices and at constant concentrations in other cases. In general, the performance of the devices falls when the $\mathrm{Ga} /(\mathrm{In}+\mathrm{Ga})$ contents exceed $\sim 0.50$, which is not understood. A comparison of the performance of the best small-area CIGS solar cell (18.8\%) with that of the best Si solar cell $(24 \%)$ is shown in Figure 1. It can be seen from the quantum efficiency data that the majority of the difference in performance is in the blue end of the spectrum. Further refinements of the device and development of novel materials for the front contact could potentially reduce or eliminate this difference, further enhancing the CIGS cell performance. One important consideration in making this comparison is that the Si cells are made by a photolithography process that is difficult to scale to large volumes; in contrast, the CIGS(S) cell is made by standard thin-film deposition methods that are currently being expanded to larger areas. In spite of this remarkable performance, factors limiting reproducibility in production of the best devices remain poorly understood.

Some of the major outstanding issues in the materials science and electrical engineering of CIGS(S) devices include the following. 


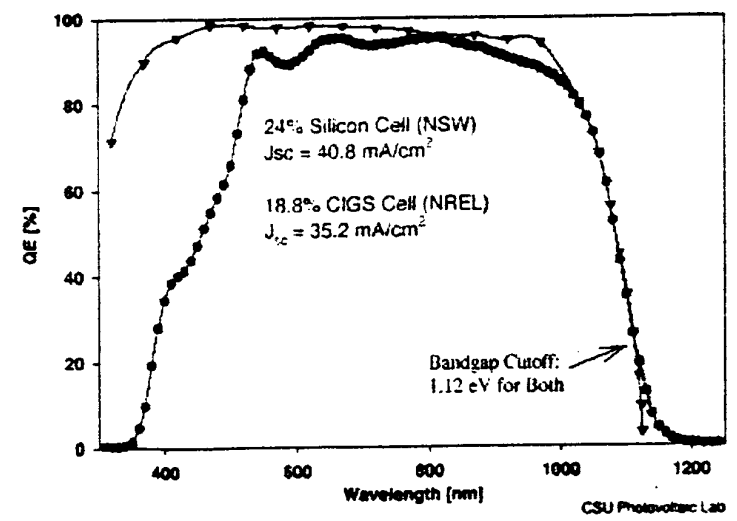

Figure 1: Compares the spectral response (left) and current/voltage (right) curves for the best CIGS solar cell with the best single-crystal Si cell.

- The primary materials factors (phases present, defects, impurities, surfaces, alloy, etc.,) that limit the performance of both record and standard devices are not known.

- The nature of the current-collecting barrier (homojunction-like or heterojunction-like) is not known.

- A detailed and predictive model of even general aspects of device performance is not available.

- All materials in the device show a clear need for improvement. This includes the front contact, the rear contact, the heterojunction-forming material, and the absorber layer itself.

- The materials and processes currently available are not compatible with fabrication of topjunction devices in tandem- structure CIGS(S) solar cells.

In addition to these areas where science can provide a more systematic procedure for improving the technology of the devices, the limits to performance of existing devices are currently set by a lack of control of the manufacturing processes. Thus, the performance of large-area devices falls significantly below the average performance of small areas of the same units. This reflects problems with yield and is closely coupled to the lack of diagnostic tools that could assist in manufacturing. Although the design and implementation of these tools for individual processes is considered by some to be "engineering" rather than "science," the development of the basic methods used in the characterization of the material and the proof of the concept to be used in process control requires significant true science to be done.

\section{BASIC RESEARCH CHALLENGES IN CIGS(S)}

\section{II.A. Major Topical Areas for Basic Research}

A prioritized list of research topical areas was developed during the course of the workshop. Each topic was assigned a consensus priority for research effort representing the fraction of total effort that the team felt should be devoted to that topic. It was assumed that all topics would be studied in parallel, and all were considered important to the ultimate success of chalcopyritebased devices. The topical areas, in order of importance and assigned priorities, were: 
Topic

I Development of an integrated predictive understanding of CIGS(S) materials and devices.

II Development of novel deposition techniques and characterization of $\quad 30 \%$ the mechanisms of growth in existing and novel processes.

III Novel materials, especially with wide energy gaps $(1.7 \mathrm{eV})$ other than $\quad 15 \%$ CIGS(S) alloys.

IV Development of real-time material characterizations for process control. $\quad 15 \%$

$\mathrm{V}$ Alternative front- and rear-contact materials.

$10 \%$

As can be seen from the priorities assigned, these topical areas fell into three ranges of importance. The first two were considered the highest priority and vital to effective engineering of advanced materials and devices. In particular, Topic I encompasses a set of detailed theoretical and experimental studies needed to resolve long-standing debates over how the materials and devices function (see discussion below).

Although tandem-junction devices are not specifically mentioned in the above list, it was clear from the discussion that these represent an important area of research. Specific issues needed to produce a working tandem-junction device based on chalcopyrite absorbers fall into Topics I, II, III, and V.

It should be noted that while the five topics identified are quite broad, they do not include all topics of interest. Section II.C. discusses some of the potential research areas that were considered interesting and potentially worthy of funding, but that did not fall into the highestpriority areas. Areas identified as primarily "engineering" rather than "science" topics were not included in the list above, but also represent important areas of research.

\section{II.B. Discussion of Topics of Research Recommended}

II.B.1. Development of an integrated predictive understanding of CIGS(S) materials and devices. The most pressing problems in the design of CIGS(S) solar cells and processes result from the lack of a detailed understanding and models of the materials and cells. Experimental and theoretical work is needed to determine assumptions to be built into device models. The objective of modeling should be to build a three-tier structure. First, basic theoretical studies using first-principles calculations are needed as a foundation for device models. Second, detailed models of the devices need to be shown to predict as many aspects of device behavior as possible. Several versions of such models exist, but they themselves, their input assumptions, and their parameters still require refinement. Finally, a simple device model providing predictiveness without intensive calculations is needed for application at the manufacturing design stage.

First-principles theoretical studies are useful primarily for two reasons: (1) Theory can isolate one factor at a time (e.g., study one particular defect, or one particular surface), whereas this is difficult experimentally. [The inability to separate experimental variables has proven a major problem in characterization of CIGS(S).] (2) Theory can consider geometries or structures that are conceivable, but currently difficult to make in the laboratory. First-principles electronic structure calculations (such as density functional theory) are important tools. In the past, most of the calculations were performed for pure chalcopyrite compounds and simple chalcopyrite alloys and interfaces. Past accomplishments include the analysis of band structures and interband optical transitions of chalcopyrites and their ordered defect compounds (ODC), order-disorder transitions of chalcopyrites and chalcopyrite/II-VI alloys, X-ray structure factors, charge density maps, band offsets between chalcopyrites and between chalcopyrites and II-VI compounds, and band-gap 
bowing of chalcopyrite alloys. However, only recently, due to a series of theoretical and computational developments, it has become possible to apply first-principles quantum theory to predict the defect properties of ternary chalcopyrite compounds. This has provided new insights into defect physics in CIGS(S). For example, these calculations investigated the effects of Ga and $\mathrm{Na}$ doping in $\mathrm{CuInSe}$, and, along with experimental results, have begun to explain the unusual defect properties in $\mathrm{CuInSe}$.

Despite recent advances in understanding of the material properties of $\mathrm{CuInSe}_{2}$ and related compounds, there are still many fundamental questions that need to be answered. It will be important in future work to examine materials from as broad a range of laboratories as possible, produced under as wide a range of conditions as possible.

Although not a complete list by any means, some of the important topics needing more work include the following:

- Understanding the relationship among surface energies and structures, the formation, structure, chemistry and growth of grain boundaries in CIGS(S), and how these affect electronic properties CIGS(S). In particular, debates concerning segregation of any of the constituent elements to the heterojunction or grain boundaries must be settled.

- Developing effective strategies for doping of CIGS(S) by extrinsic impurities including determination of solubility, level-depth, whether co-doping can improve the dopability in CIGS(S), and which intrinsic defect limits the maximum doping in $\mathrm{CuInSe}_{2}$. It will also be useful to determine the defects controlling the lifetime of minority carriers and the trapping and detrapping rates for these.

- Developing improved alloys, defect phases, and polytypes of $\mathrm{CuInSe}_{2}$ that can improve the PV properties.

- Determining whether the solar cells should be viewed as homojunction or heterojunction devices, and determine the role of the window/heterojunction materials.

- Establishing a data base for all chalcopyrite compounds, and use it to select the best materials for absorbers, multijunction partners and window materials.

- Developing a user-friendly device model using the first-principles model results, as well as experimental data, as input to predict the efficiency of the solar cells.

II.B.2. Characterization of film growth mechanisms and development of novel deposition techniques. The development of photovoltaic device structures based on CIGS(S) has advanced rapidly during the last few years. The direct energy gap of CIGS(S) results in a large optical absorption coefficient, which permits the use of thin layers $(1-2 \mu \mathrm{m})$ of active material, and allows high-device performances in spite of the modest carrier diffusion lengths. The highestefficiency CIGS(S) device was prepared based on a multi-step physical vapor deposition (PVD) process in which elements are simultaneously co-deposited onto the substrate. Unfortunately, PVD is challenging to scale up in both deposited area and throughput. Process temperatures also tend to be high, which complicates use of polymer substrates and permits interdiffusion of layers in superstrate device geometries. Thus, there is a need to develop novel low-cost and lowtemperature methods to form CIGS(S) films. A related high-priority issue in CIS PV technology is improving the understanding of thin-film growth mechanisms in both traditional and novel processes. It is good news that the quality of the CIGS(S) films and devices is becoming increasingly decoupled from the method of film delivery because of improved understanding of growth. However, much work remains to be done. 
Vapor-phase co-deposition of elements deposits the chalcopyrite film atom-by-atom with great precision. There are a number of unresolved questions about how PVD co-deposition parameters (e.g., substrate temperature, total- and differential-atomic fluxes, background ambient, and pressure) might be optimized to further improve CIGS(S) film quality. Multi-step sequential vapor-phase processes are arguably better suited than vapor-phase co-deposition to large-scale, low-cost manufacturing. However, much remains to be understood about them. For example, the mechanisms through which Group I-III metal alloy layers react with Group VI elements to form dense, large-grain CIGS(S) films that adhere well to an underlying electrode are incompletely studied. The mechanisms by which the substrate and electrodes affect the characteristics of resulting CIGS(S) films, and how one might optimally select substrates/electrodes and/or tailor CIGS(S) film deposition processes to surrounding materials need work. Optimal use of alkali metals to improve film properties and achieving desired composition grading in multi-step processes would also benefit process designs. Given the industrial importance of vapor-phase codeposition and multi-step processes, there is great leverage in their better understanding.

Vapor-phase co-deposition and multi-step processes have in common the use of vacuum deposition methods, which have numerous advantages, including precision composition control, a broad base of processing know-how, and a knowledge base for design of research and production-scale equipment. However, vacuum deposition suffers from high up-front capital equipment costs. A variety of novel film-deposition methods promise to replace capital-intensive physical vapor deposition with non-vacuum deposition of constituent layers and/or complete CIGS(S) films.

Currently, a great deal of effort is being made to develop such low-cost technologies. Processes based on electrodeposition (ED), electroless-deposition (EL), and nanoparticle-based processes have the potential for: (a) a low-cost, high-rate synthesis of CIGS(S); (b) large-area, continuous, multi-component, low-temperature depositions; (c) non-vacuum, low-capital-cost processes; and (d) efficient material use. The devices fabricated using ED, EL, and nanoparticle precursors resulted in efficiencies of $15.4 \%, 13.4 \%$, and $12.4 \%$, respectively. Such novel techniques offer a variety of potential advantages, but in general they are much less mature -- both from the standpoint of basic science understanding and from the standpoint of empirically developed standard recipes. For example, the mechanisms of nanoparticle layer densification and grain growth are not yet clear. As with studies of vacuum processes, there is significant leverage in exploring non-vacuum processes in more detail.

II.B.3. Novel materials. Novel materials based on ternary CuInSe $\mathrm{C}_{2}$ hold promise for further increasing device efficiencies and lowering product costs. Of particular interest are wider-bandgap materials suitable as absorber films in multijunction devices. Isovalent alloys with gallium and sulfur are useful in this regard, and improved understanding of these materials is a research priority. To date, modest additions of $\mathrm{Ga}$ and $\mathrm{S}$ in $\mathrm{CuInSe} \mathrm{Se}_{2}$ have been shown to increase singlejunction device efficiencies. However, considerable work is needed to make wide-band-gap materials work as well as currently employed narrow-gap compounds. Furthermore, novel materials, including non-isovalent, non-isostructural alloys such as CIS-ZnSe and nanocrystalline and multi-phase materials may provide advantages relative to the CIGS(S) alloys explored to date. Of particular value would be wide-bandgap materials that can yield efficient superstrate cells that can be mechanically stacked with existing substrate cells to form tandem junctions. Materials that can be processed at low temperatures would facilitate the fabrication of monolithic tandem structures. Further work in characterizing such new materials and exploring which materials might be useful in very high-efficiency multijunction structures should be a priority. 
II.B.4. Development of real-time material characterizations for process control. A major issue in any manufacturing environment is the ability to assess the product performance non-destructively during processing. The goals of any such analyses are first to produce material within given tolerances reproducibly. Second, it is necessary to control uniformity and defects in the devices to increase product yield and performance. To be most effective, the test should be nondestructive so that it can be used on all devices. It should not be performed on occasional test structures. Rather, it should take place at many points during product formation on the normal production material to reduce continued manufacturing on already unsatisfactory devices. Finally, an in-situ analysis should permit feedback control of the process to correct problems as quickly and effectively as possible.

The first problem in designing process control systems is knowing what to test. As described above, major questions remain concerning what is characteristic of a good device. General composition ranges can be specified, but many other issues such as the best surface morphology, crystalline orientation, and microstructure are matters of intense debate. Thus, achievements in this category of basic research will be aided by progress in the other topical areas listed here. Nonetheless, some general recommendations can be made.

It is generally agreed that the optimal composition of CIGS(S) films is near stoichiometry, but slightly to moderately rich in the group III elements. The exact amount by which the film should be III-rich is still controversial. Some in-situ, non-destructive, non-contact strategies for composition control have been reported. In addition, uniformity and defect density in devices have been studied using optical-beam-induced current (OBIC) measurements. Although this is not a non-contact method and is used at the end of the process, it has been useful in quickly characterizing defects in large areas of devices. A number of other process control strategies have been considered. However, there is clearly a need for much more sophisticated techniques. Examples of such methods could include applications of X-ray fluorescence spectroscopy, photoluminescence, ellipsometry, resistivity, laser scattering, and dual-beam optical modulation (DBOM). Results have been reported with all of these methods characterizing the CIGS(S) materials and devices. However, none have been effectively exploited for process control.

Successful development of effective process control strategies requires detailed scientific analysis of CIGS(S) materials using the techniques and a careful quantitative study of the results to show how specific conditions can be detected and maintained. To control processes effectively, it is necessary to understand the physics of the analysis methods. Relatively little effort has thus far been devoted to understanding the methods outside of manufacturing environments. What has been done has been largely empirical and generally does not produce the basic understanding needed to truly design new methods of process control.

II.B.5. Alternative front- and rear-contact materials. Current CIGS(S) devices use Mo for the back contact and $\mathrm{CdS}$ combined with $\mathrm{ZnO}$ as the front contact. It is widely agreed that none of these materials is satisfactory. Mo retains large amounts of stress under typical deposition conditions. This is particularly awkward when using flexible substrates. Adhesion of Mo both to the glass substrate and to the CIGS(S) film can be a problem and can lead to localized or general failures. The surface chemistry and reactivity of Mo with Se can influence the characteristics of the absorber and the performance of the device. For example, the presence of a $\mathrm{Mo}_{\mathrm{x}} \mathrm{Se}$ interlayer between the absorber and the Mo can reduce the penetration of $\mathrm{Na}$ from the substrate glass into the absorber. This, in turn, can decrease the acceptor concentration and voltage output of the device. Furthermore, Mo is opaque and consequently will be unacceptable for the back contact of the top device in any tandem-junction device. 
The current heterojunction partner material, CdS, is unattractive because of the environmental hazards associated with $\mathrm{Cd}$. Furthermore, although efficient CIGS(S)-based devices are routinely fabricated using a very thin CdS "buffer layer," uncertainty remains as to why solution-deposited $\mathrm{CdS}$. There is also the question of whether alternative materials (e.g., ones that don't contain $\mathrm{Cd}$ lowers the efficiency of the device) and alternative processes (e.g., those compatible with in-line vacuum processing) might provide equal or better performance. Consequently, new contact technologies are needed for both single and multijunction CIGS(S) devices.

The next-generation CIGS(S)-based thin-film device will ideally have the following characteristics. The back-contact and substrate combination will offer superior reproducibility to the present soda-lime glass/Mo or stainless-steel/Mo systems. This will expand the list of potential substrates and back-contact metals to those more optimally suited to CIGS(S) thin-film processing and will reduce the cost of manufacturing. Considerable research will be required to understand the details of the interaction of contacts with the absorber layers and how to improve the contact designs.

\section{II.C. Other Issues}

Although the most pressing issues in the science of chalcopyrite-based solar cells are covered above, other areas are also worthy of research support. For example, even though CIGS(S)-based devices are well-known for their stability and CIGS(S)-based prototype products have impressive durability, CIGS(S) devices can show transient effects related to electrical bias, light exposure, encapsulation, etc., that require explanation. Although CIGS(S)-based PV devices are relatively impervious to environmental exposure, long-term product performance in a wide range of demanding applications requires a durable, low-cost package. CIGS(S)-based PV shares with other PV technologies a common need for better, more-stable potent materials and less-expensive transparent cover materials. Many other issues could be listed here and are omitted only for brevity. CIGS(S) remains the solar cell technology most in need of studies of a broad range of issues both in science and engineering.

\section{SUMMARY AND CONCLUSIONS}

The team concludes that a well-planned program of basic scientific research can have a major impact on both the viability of the current-generation CIGS(S)-based solar cells and on the design and implementation of future-generation chalcopyrite devices. We have identified five topical areas of major importance to this process and have attempted to outline some of the specific experiments that could be performed and models that should be developed to have the maximum impact in this field. We have recommended a mixture of approaches aimed at both evolutionary and revolutionary progress. The purpose of the scientific research should be to develop quantitative deposition process/device performance relationships that can be used for process design optimization and control, as well as in the practical possibility of non-empirical engineering of future-generation devices.

\section{ACKNOWLEDGEMENTS}

The authors thank J. F. Hiltner and J. R. Sites of Colorado State University for preparing Fig. 1. The authors also acknowledge the support of the Department of Energy and the National Renewable Energy Laboratory. 


\title{
CRITICAL ISSUES AND RESEARCH NEEDS FOR CdTe-BASED SOLAR CELLS
}

\author{
A. D. Compaan ${ }^{1}$, J. R. Sites ${ }^{2}$, R. W. Birkmire ${ }^{3}$, C. S. Ferekides ${ }^{4}$, and A. L. Fahrenbruch ${ }^{5}$ \\ ${ }^{1}$ University of Toledo, Dept. of Physics, Toledo, Ohio 43606 \\ ${ }^{2}$ Colorado State University, Dept. of Physics, Fort Collins, Colorado 80523 \\ ${ }^{3}$ Institute of Energy Conversion University of Delaware, Newark, Delaware 19716 \\ ${ }^{4}$ University of South Florida, 4202 E. Fowler Avenue, Tampa, Florida 33620 \\ ${ }^{5}$ ALF, Inc., 107 Montalvo Road, Redwood, California 94062
}

\begin{abstract}
We present a review of the basic features of polycrystalline thin-film CdTe solar cells and modules and then survey the outstanding fundamental issues and research challenges for application of this materials system to photovoltaic devices.
\end{abstract}

\section{INTRODUCTION}

Polycrystalline thin-film cadmium telluride (CdTe) solar cells are one of the most promising candidates for large-scale application of photovoltaic energy conversion because they have shown laboratory efficiencies in excess of $15 \%{ }^{1,2}$ and module performance of over $10 \%{ }^{3}$ In addition, CdTe solar cells with efficiencies over $11 \%$ have been made by a variety of deposition methods ${ }^{4}$, and several of these methods have been adapted to module manufacturing. Several good reviews exist of the deposition methods as well as module and device performance. ${ }^{5-7}$

Progress towards improving device and module performance has been slow over the past five years and the translation of laboratory results to first-time manufacturing has been more difficult than expected due to the complexity of the processes involved for making large area, thin-film CdTe modules. The slow progress is further compounded by the lack of the fundamental scientific and engineering knowledge base required to underpin the development of the technology. In this paper, critical issues are identified that have impeded the advancement of CdTe solar cell technology, and areas of basic research opportunities are identified.

All high-efficiency CdTe/CdS devices have been fabricated in a superstrate configuration as shown in Figure 1, where the $\mathrm{CdS}$ and the $\mathrm{CdTe}$ are deposited sequentially onto a glass/transparent-conducting-oxide (TCO) superstrate. Although there are variations in device fabrication process, particularly with respect to how the CdTe is deposited, it is possible to describe a "generic" fabrication procedure for high efficiency solar cells:

1. A glass superstrate is first coated with a TCO such as $\mathrm{SnO}_{2}$ or indium tin oxide (ITO). The choice between ITO and $\mathrm{SnO}_{2}$ is primarily determined by the deposition temperature of $\mathrm{CdS}$ and/or CdTe films. For low temperature $\mathrm{CdS}$ and CdTe deposition processes, ITO is the material of choice, having higher optical transmission for a given sheet 


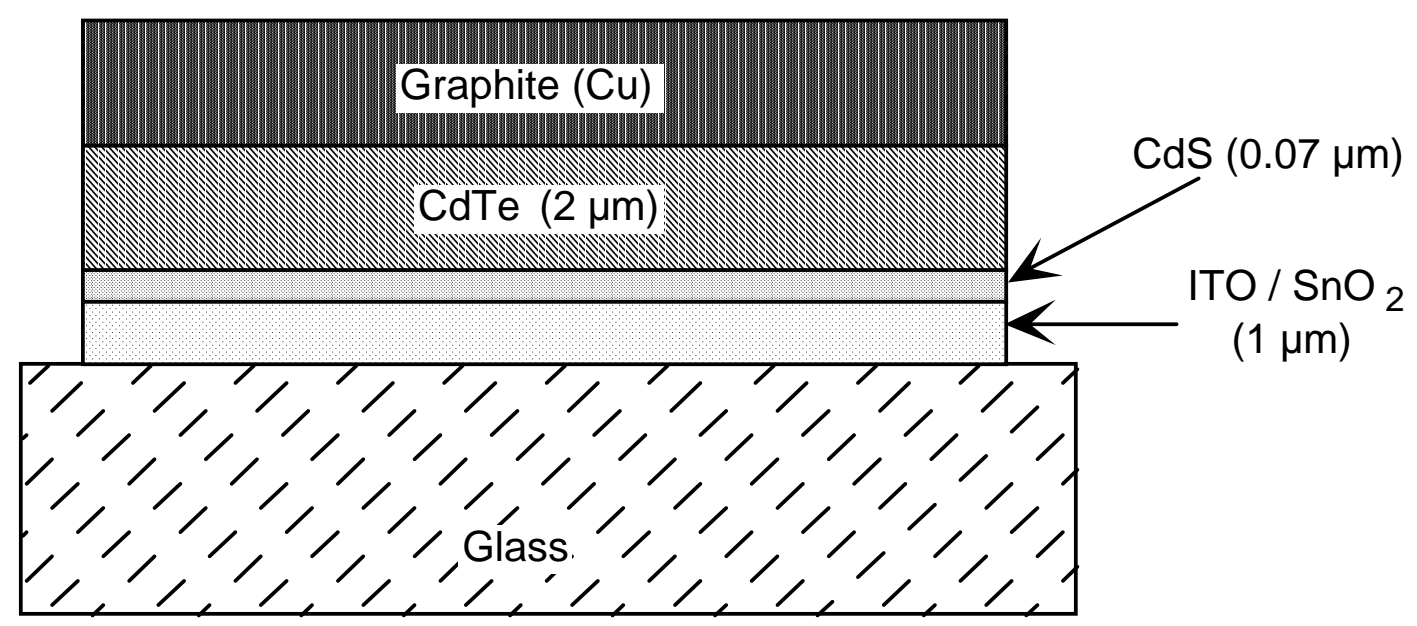

Figure 1. Basic CdS/CdTe cell structure.

resistance. For $\mathrm{CdS}$ and/or $\mathrm{CdTe}$ deposition processes requiring high temperatures, $\mathrm{SnO}_{2}$ is the best material since it is more stable, mainly due to the fact that the deposition process itself requires temperatures of $450-550{ }^{\circ} \mathrm{C}$.

2. The CdS film is deposited by a wide variety of processes including close-spaced sublimation (CSS), vapor-transport deposition (VTD), chemical-bath deposition (CBD), sputtering, screen printing, electrodeposition, atomic-layer epitaxy (ALE), physical-vapor deposition, and spray pyrolysis. For thin $\mathrm{CdS},<100 \mathrm{~nm}$, the film is typically heat treated in a reducing atmosphere or in the presence of $\mathrm{CdCl}_{2}$. The heat treatment increases grain size and may reduce the defect density of the films. ${ }^{8-11}$

3. The CdTe film is deposited by a process such as close-spaced sublimation, screen printing, spraying, electrodeposition, atomic-layer epitaxy, physical-vapor deposition, sputtering, chemical-vapor deposition, molecular-beam epitaxy, or laser ablation. Substrate temperatures are from $80^{\circ} \mathrm{C}$ to about $630^{\circ} \mathrm{C}$ depending on the deposition process and the CdTe films are from $2 \mu \mathrm{m}$ to $10 \mu \mathrm{m}$ thick.

4. The $\mathrm{CdTe} / \mathrm{CdS}$ structure is typically given a post-deposition heat treatment while exposed to $\mathrm{CdCl}_{2}$ or another $\mathrm{Cl}$ containing compound such as $\mathrm{Cl}_{2}$ or $\mathrm{HCl}{ }^{12,13}$ In some cases, such as in electrodeposition and screen printing, the chlorine may be contained in the original CdTe deposit. ${ }^{14,15}$ The heat treatment enables grain growth, reduces defect density in the films, and promotes the interdiffusion of the CdTe and CdS layers. ${ }^{16-18}$ The degree of diffusion varies among the various deposition procedures, but, for equilibrium conditions, it is limited by the miscibility gap between CdS and CdTe. ${ }^{17,18}$ In all cases the asdeposited thickness of the CdS layer is reduced and the $\mathrm{CdS} / \mathrm{CdTe}$ interface becomes alloyed into a $\mathrm{CdTe}_{\mathrm{x}} \mathrm{S}_{1-\mathrm{x}} / \mathrm{CdS}_{\mathrm{y}} \mathrm{Te}_{1-\mathrm{y}}$ interface, where $x$ and $y$ are less than or equal to the solubility limits at $\sim 400{ }^{\circ} \mathrm{C}(\mathrm{x} \sim 0.03$ and $\mathrm{y} \sim 0.06) .{ }^{18}$

5. There is a wide diversity in structures and procedures for electrical contacts that generally includes at least two layers: the primary layer is a heavily doped or degenerate layer that makes a low-loss electrical contact to the CdTe; and the secondary contact is metal or conductive ink that carries the current laterally. Most fabrication procedures include: an etch or surface preparation step, which may produce a Te-rich surface layer and remove oxides; creation of the primary layer, either by deposition of a $\mathrm{p}^{+}$-layer or $\mathrm{ZnTe}: \mathrm{Cu}, \mathrm{HgTe}$ 
or $\mathrm{PbTe}$ or by modification of the CdTe surface by supplying a p-type dopant; a subsequent heat treatment above $150{ }^{\circ} \mathrm{C}$; and application of the secondary contact by sputtering, vacuum evaporation or screen printing. ${ }^{19-25}$

From a device perspective, the thin-film $\mathrm{CdTe} / \mathrm{CdS}$ heterojunction devices show very lowminority carrier recombination at the absorber grain boundaries and at the metallurgical interface which results in high quantum efficiencies. However, open-circuit voltages are relatively low due to recombination in the space charge region in the CdTe.

\section{MATERIALS AND DEVICE PROPERTIES}

In this section, we provide some additional details of the CdTe-related materials and devices with the objective of illustrating some of the complexity of these cells and describing some of the outstanding fundamental challenges that we believe impede progress in CdTe-based cell performance.

\section{Device Model}

The ideal one-dimensional device structure of Figure 1 may apply nicely to junctions in crystalline or epitaxial thin-film devices; however, it ignores the effects of grain boundaries and a variety of other complicating phenomena of the polycrystalline thin-film $\mathrm{CdS} / \mathrm{CdTe}$ solar cell. A more realistic device structure is sketched in Figure 2a where the

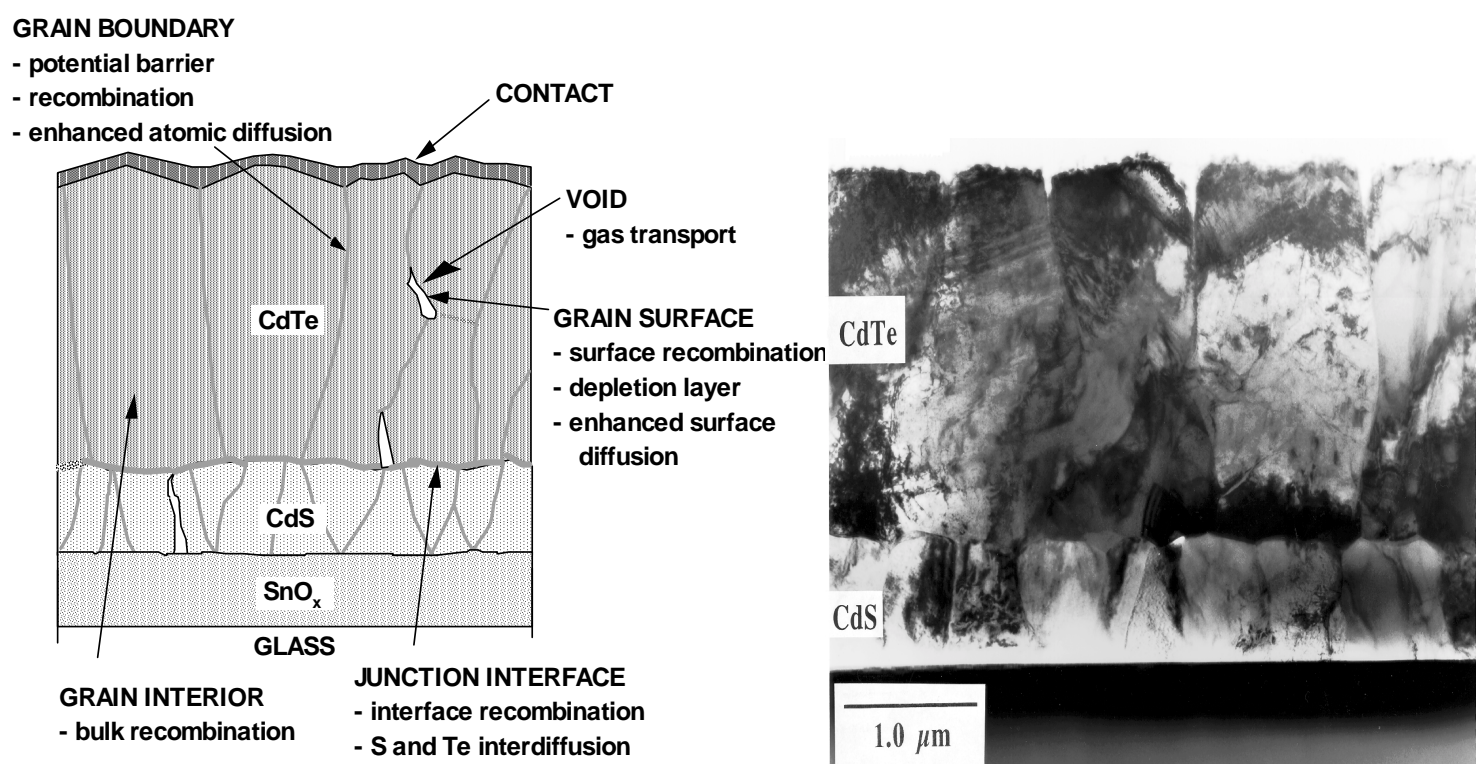

Figure 2. a) CdS/CdTe cell structure showing polycrystallinity and related issues; b) TEM cross-sectional micrograph of a typical cell.

grain boundaries are displayed for $\mathrm{CdS}$ and CdTe layers. Note that there is a lattice mismatch of slightly greater than $10 \%$ at the heterojunction ${ }^{26}$ so that epitaxial growth is necessarily limited. Successful device fabrication requires thorough understanding of, and ability to control, the effects of grain boundaries, internal voids, possible pinholes through the thin CdS layer, interdiffusion across the $\mathrm{CdS} / \mathrm{CdTe}$ interface, and the establishment of a low 
resistance contact without degrading the junction properties. The TEM cross-sectional micrograph of Figure $2 \mathrm{~b}$ illustrates the validity of this structural model.

Figure 3 illustrates a structure with additional complexity, some aspects of which are certainly present. There is compelling evidence of interdiffusion at the CdS/CdTe interface forming a sulfur-rich layer of $\mathrm{CdTe}_{\mathrm{y}} \mathrm{S}_{1-\mathrm{y}}(\mathrm{y} \sim 0.03)$ and a Te-rich layer of $\mathrm{CdS}_{\mathrm{x}} \mathrm{Te}_{1-\mathrm{x}}(\mathrm{x} \sim 0.06) .{ }^{16,18}$ It has been proposed that this interdiffusion process results in Srich spikes along the grain boundaries. This S-rich material is likely to be n-type and could lead to enhanced electron collection from the CdTe.

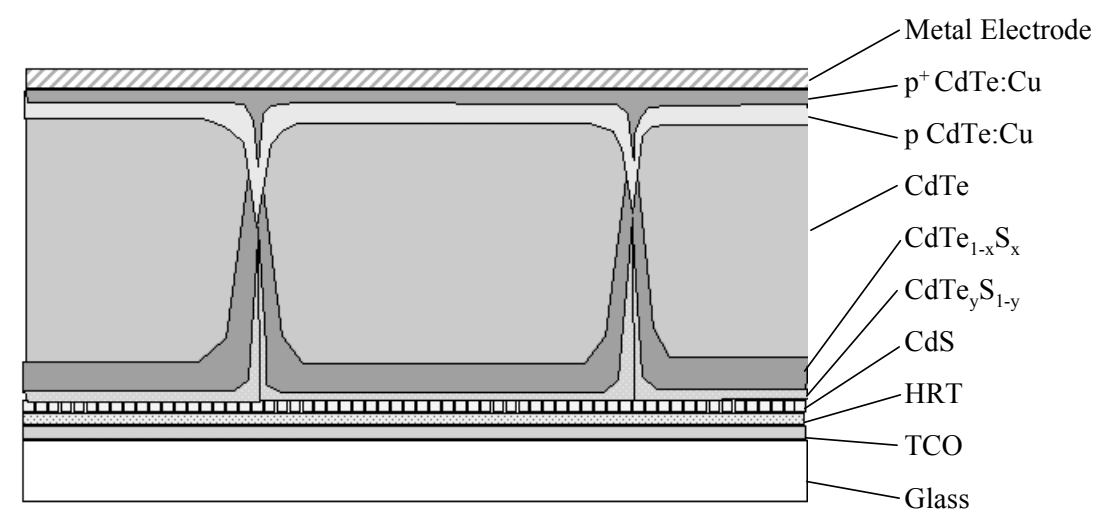

Figure 3. Further details of $\mathrm{CdS} / \mathrm{CdTe}$ cell structure showing possible three dimensional effects. (From P. V. Meyers.)

In addition, the contacting process for the back of the cell cannot be assumed low resistance. It most commonly involves the creation of a heavily doped p-layer under the metal or graphite back contact. Such contacts sometimes produce a potential barrier high enough to produce non-negligible losses and can have stability problems. Nevertheless, more than one type of back contact has been shown to be sufficiently low resistance and to have excellent stability under cell ${ }^{28}$ or module ${ }^{3,29}$ operating conditions. Finally, Figure 3 shows the presence of a high-resistivity tin oxide (HRT) layer between the TCO and CdS layers. A variety of HRT layers have been used ${ }^{30-32}$ and have been shown to improve device yield and may also enhance stability for some structures and under some stress conditions. Further discussion of the HRT layer is given later.

\section{$\underline{\mathrm{CdS} / \mathrm{CdTe} \text { Interdiffusion }}$}

Some interdiffusion of $\mathrm{S}$ and Te across the metallurgical junction probably occurs during film growth, especially for the high temperature deposition processes. However, further interdiffusion occurs during the post-deposition treatment near $400^{\circ} \mathrm{C}$ in the presence of $\mathrm{CdCl}_{2}$ or vapors containing $\mathrm{Cl}^{12,13}$ In the blue from $400 \mathrm{~nm}$ to $500 \mathrm{~nm}$, absorption in $\mathrm{CdS}$ attenuates the light reaching the CdTe layer, and thus reduces the quantum efficiency $(\mathrm{QE})$ of the cell in this region. (No carrier collection appears to occur in the $\mathrm{CdS}$, either because the minority carrier (hole) lifetime is too low or because other factors present barriers to hole collection.) Note in Figure 4 that, as the CdS thickness is reduced from $100 \mathrm{~nm}$ to $40 \mathrm{~nm}$ and to zero, the QE in the blue rises. 


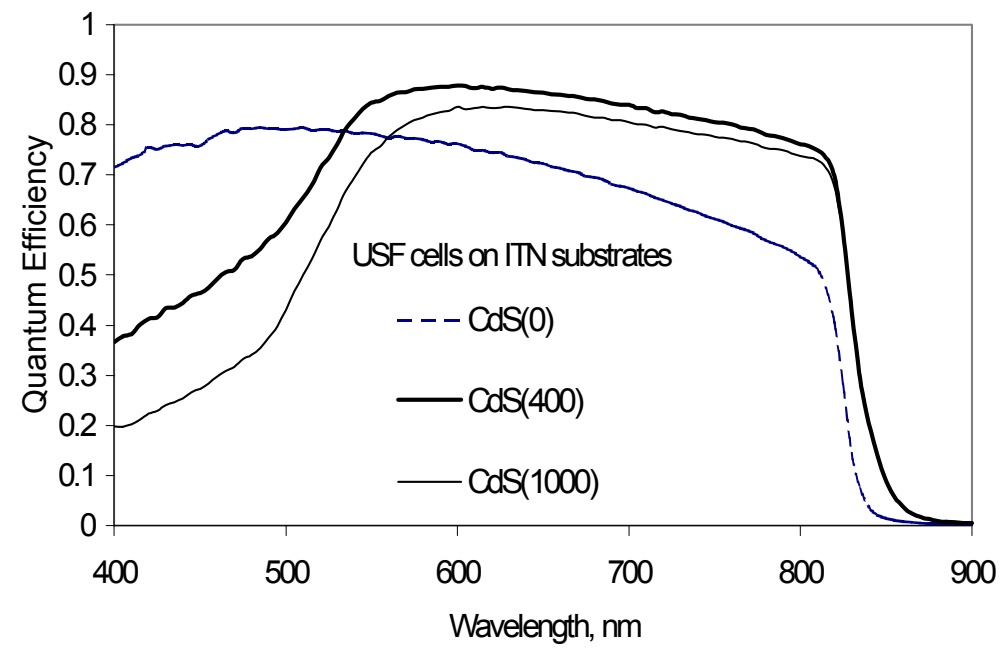

Figure 4. QE with and without CdS layers on substrates with an HRT layer as sketched in Figure 3. Soda-lime glass/ $\mathrm{SnO}_{2}$ substrates from LOF; HRT deposited at ITN; cells fabricated at University of South Florida.

The QE of Figure 4 also shows evidence of the S-Te interdiffusion. The reduced QE between $520 \mathrm{~nm}$ and $580 \mathrm{~nm}$ provides prima facie evidence of interdiffusion of Te into the CdS. (The band gap shrinks at a rate of $\sim 2.7 \mathrm{eV}$ per unit $y$ with increasing Te content in the $\mathrm{CdTe}_{\mathrm{y}} \mathrm{S}_{1-\mathrm{y}}$. ${ }^{17,33}$ Further evidence of interdiffusion arises from the red edge of the spectral response. When cells are fabricated with no CdS layer, the red edge of the $\mathrm{QE}$ lies at the band edge of pure CdTe $(\sim 820 \mathrm{~nm})$. However, when cells are fabricated with some CdS, part of the CdS layer is consumed and the QE extends an additional $10 \mathrm{~nm}$ to $20 \mathrm{~nm}$ into the near infrared-in some cases up to $850 \mathrm{~nm} .{ }^{30}$ This is consistent with sulfur diffusion into CdTe, since the $\mathrm{CdTe}_{1-y} \mathrm{~S}_{\mathrm{y}}$ alloy exhibits large band bowing. ${ }^{17,33}$ Other convincing evidence of the interdiffusion has been obtained with grazing angle x-ray diffraction. ${ }^{18}$

It should also be noted from the QE curves of Figure 4 that the absence of an initial CdS layer reduces the overall quantum efficiency. There is some evidence that the use of an appropriate high-resistivity TCO buffer layer (HRT) between the low-resistivity TCO and the CdTe can permit the reduction of the CdS layer thickness to zero while maintaining high $\mathrm{V}_{\mathrm{OC}}$, provided that a low-sulfur alloy, $\mathrm{CdS}_{\mathrm{x}} \mathrm{Te}_{1-\mathrm{x}}$, is used at least near the HRT interface. ${ }^{30}$

\section{Back Contacts with Copper}

One of the most challenging aspects to the fabrication of high performance CdTe cells is the achievement of low-resistance back contacts. The typical contact employs $\mathrm{Cu}$ in some way, presumably because the $\mathrm{Cu}$ diffuses into the CdTe, producing a heavily doped $\mathrm{p}$ layer. This has been accomplished by diffusion of $\mathrm{Cu}$ into $\mathrm{CdTe}$ and then deposition of a metal or carbon paint. 19,21,23,24 Alternatively, the copper may be introduced by mixing $\mathrm{HgTe}: \mathrm{Cu}$ into the carbon paint which is then baked at temperatures up to $300^{\circ} \mathrm{C}$ where some diffusion may occur. ${ }^{20,25}$

As long as $\mathrm{Cu}$ is employed in the back contact structure, it will be critical to understand and control the role of $\mathrm{Cu}$ in $\mathrm{CdTe}$. Some of the variety of effects are illustrated in the 
photoluminescence spectra shown in Figure 5. These data were obtained on single crystal $\mathrm{CdTe}$ at $10 \mathrm{~K}$ but similar, although broader, features are observed from polycrystalline films. ${ }^{34,35}$
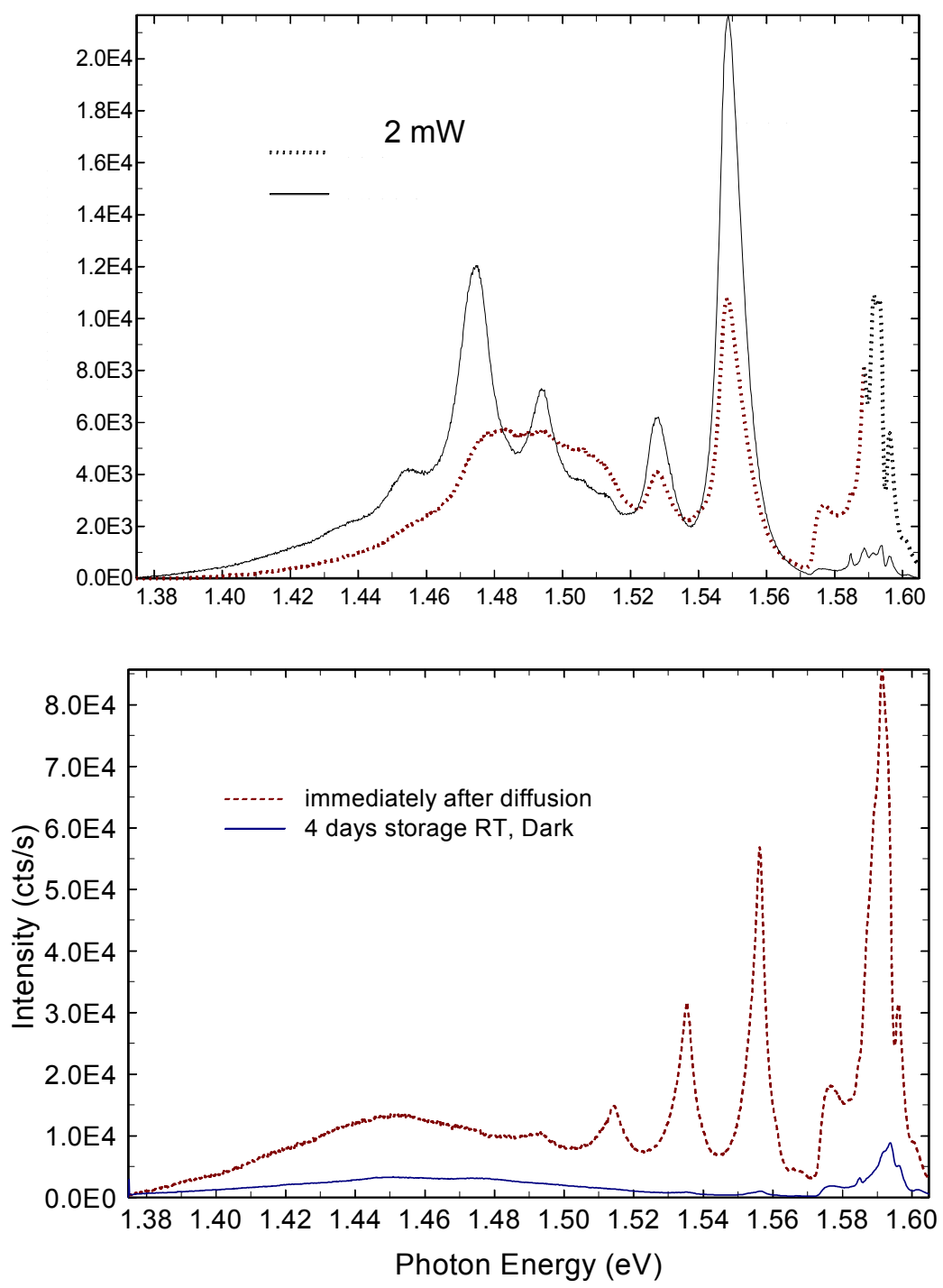

Figure 5. PL from crystalline CdTe at $10 \mathrm{~K}$ excited at $752 \mathrm{~nm}$. a) Pure CdTe showing intensity dependence; b) copper doped CdTe showing changes under 300 K storage. $2 \mathrm{~mW}(0.08 \mathrm{~mW})$ corresponds to about $10(0.4)$ suns in the $0.5 \mathrm{~mm}$ spot.

Three types of features can be distinguished in the spectra: free and bound exciton-related peaks $(1.575 \mathrm{eV}-1.60 \mathrm{eV})$, donor-acceptor (D-A) pair transitions from relatively shallow states $(1.51 \mathrm{eV}-1.56 \mathrm{eV})$, and deeper lying D-A transitions $(1.40 \mathrm{eV}-1.50$ eV). ${ }^{36,37}$ The spectra depend strongly on the excitation power with exciton-related features dominating at high excitation powers due to the inherent bimolecular recombination. (See Figure 5a.) At very low power, however, the D-A pair transitions dominate. Note that the transition near $1.55 \mathrm{eV}$ shifts slightly to the blue with increasing excitation power. This is characteristic of D-A pairs due to the fact that the formation 
rate, the recombination rate, and the final state Coulomb energy (of the ionized donor and ionized acceptor) are dependent on the pair separation in the lattice. ${ }^{38}$ Diffusion of $\mathrm{Cu}$ into CdTe quenches the shallow D-A pair (probably $\mathrm{D}-\mathrm{V}_{\mathrm{Cd}}$ ) transition at $1.55 \mathrm{eV}$ but produces a new peak near $1.45 \mathrm{eV}$ (presumably $\mathrm{D}-\mathrm{Cu}_{\mathrm{Cd}}$ ). Also $\mathrm{Cu}$ doping produces a dominant bound exciton peak near $1.59 \mathrm{eV}$ (probably $\mathrm{X}-\mathrm{Cu}_{\mathrm{Cd}}$ ). Additional $\mathrm{Cu}$-related complexes form (possibly from combinations of $\mathrm{Cu}$ interstitials $\mathrm{Cu}_{\mathrm{i}}$, cadmium vacancies $\mathrm{V}_{\mathrm{Cd}}$, and/or substitutional copper $\mathrm{Cu}_{\mathrm{Cd}}$ ), which are quite unstable and disappear with time even at room temperature. These complexes have strong lattice coupling as seen from the optic-phonon side bands in luminescence.

\section{BASIC RESEARCH DIRECTIONS}

There are several basic research issues that will quite likely have a direct impact on the continuing development of high-performance polycrystalline CdTe solar cells. At least some of these can be pursued with relatively straightforward measurement and analysis techniques. As with many polycrystalline materials systems, much of the challenge will be to develop a user-friendly shared data base from several systematic series of measurements.

\section{Polycrystalline CdTe}

The basic nature of polycrystalline CdTe has been elusive, and clearly more detailed knowledge is critical. What we know is that several deposition techniques yield similar quality solar cells, that the material used in cells has a low carrier density $\left(10^{15} \mathrm{~cm}^{-3}\right.$ range), that post-deposition annealing, generally with $\mathrm{CdCl}_{2}$, can increase both crystallite size and hole density, and that the resulting structure resembles the one shown in Figure 2. There is almost certainly a compensating process involving native defects, but at this point, there is no convincing description of the dominant process, or even strong evidence to decide whether it is associated with bulk CdTe or with granular surfaces. Theoretical evaluation of common possibilities involving vacancies, antisite doping, and simple complexes have recently been done with $\mathrm{CuInSe}_{2}{ }^{39}$ through calculation of the chemical potential as a function of Fermi level. Similar effort may provide useful insights for CdTe as well. Defect equilibrium models relating the densities of the various charged defects, dopant atoms, and free carriers have been done for $\mathrm{p}$-CdTe:P in the past, ${ }^{40}$ but the subject should be revisited with regard to $\mathrm{CdTe}$ with $\mathrm{Cu}, \mathrm{Cl}$, and $\mathrm{O}$ impurities.

Existing measurement techniques, such as photoluminescence, capacitance, DLTS, and lateral resistivity, can also be very powerful. They should incorporate systematic variation of temperature and illumination, and they should be applied to series of CdTe samples prepared with systematic variation of one or more process variables. Even better would be improvement of techniques for making such measurements locally with high spatial resolution, so that one might have direct information to separate grain-boundary effects from those of the bulk. These measurements should be extended to other series of samples made with varying concentrations of extrinsic dopants and with alloys where Te is partially replaced with $\mathrm{S}$ and $\mathrm{Cd}$ with $\mathrm{Zn}$. Virtually all solar cells show transient behavior of cell parameters to some degree. Although this has been explored for time scales of minutes to hours (e.g., for $\mathrm{CdS} / \mathrm{CuInSe}_{2}{ }^{41}$ ), valuable information might be 
obtained by examining the transient behavior of the $\mathrm{CdS} / \mathrm{CdTe}$ device on time scales from $10^{-6} \mathrm{sec}$ to several seconds as a function of temperature and light intensity. In addition, it is important to map this territory in order to be sure that more routine testing is consistent. Finally, there should be major refinements of in situ process monitoring for both the vapor constituents and the growth surface. Greater assurance that the CdTe layer is uniform and reproducible will assist the measurement systematics, and may also lead directly to better performance cells.

\section{CdS/CdTe Junction}

Traditionally, $n-C d S$ has been used to form a diode junction with p-CdTe, and the two materials, as shown in Figure 1, have usually been viewed as separate layers. There is growing evidence, as shown in Figure 3, that there is significant interdiffusion of $\mathrm{S}$ and Te in the completed diode. It is likely in fact that the degree and nature of this interdiffusion plays a major role in determining the actual junction location, diode quality, and cell performance. Again, a systematic series of samples, this time with the amount of interdiffusion used as the varied parameter, is recommended. The correlation of photovoltaic characterization with elemental profiles, morphology of the junction region, and post-deposition annealing should be helpful to our basic understanding of the diode junction.

The identification of specific dopant and recombination states in the CdS/CdTe-junction region is a difficult problem. It is also a critically important one, since these are the physical parameters that ultimately determine solar-cell performance. Theory may give some insight, but the most powerful experimental tool is likely to be photoluminescence (PL) with illumination through the CdS layer of completed cells. This configuration tends to be sensitive to the same region that controls the junction, and there have already been credible measurements of changes in peak height, peak position, local variations, and lifetime with post-deposition anneals. ${ }^{35,42,43}$ There needs to be a basic verification that PL, normally done at extremely high injection levels, indeed reflects the AM1.5 cell parameters accurately. Extension of the spectral range of PL to about $0.5 \mathrm{eV}$ might yield additional information about midgap recombination centers (and check the common assumption that all recombination is non-radiative in this material). Finally, it would be very helpful to reduce or eliminate the use of relative units in PL spectroscopy measurements, making it possible to compare samples more productively. Three very practical questions are: 1) whether industrial fabrication should be designed to explicitly incorporate a mixed $\mathrm{CdS}_{\mathrm{x}} \mathrm{Te}_{1-\mathrm{x}}$ layer; 2) whether doing so might allow the elimination of a separate $\mathrm{CdS}$ layer; and 3) whether there may be alternatives to post-deposition annealing to achieve high performance. A more basic question is how is it that $\mathrm{CdS}$ provides good junctions with CdTe. Is it poor band line-up between TCOs and CdTe? Is it passivation of recombination centers similar to the way $S$ is used for surface passivation in the III-V system? Does interdiffusion act to getter defects near the junction? Or, does three-dimensional interdiffusion of $\mathrm{CdS}$ and CdTe create a favorable geometrical structure?

A very useful framework for comparison of different $\mathrm{CdS} / \mathrm{CdTe}$ solar-cell results are computational simulations, based on carrier transport theory, using finite element 
mathematical techniques. Several user-friendly programs exist, ${ }^{44}$ but they have been developed for one-dimensional analysis of single-crystal cells and need enhancements to account for polycrystalline features and for tunneling. In many cases the issue is whether the three dimensionality inherent in a polycrystalline structure can be reduced to a more manageable problem, such as separately lumping bulk and grain-boundary effects and then treating them in parallel. Creation of user-friendly two-dimensional simulators should be a priority.

\section{Front Contact}

Essentially all CdTe solar cells rely on a transparent conductive oxide, generally $\mathrm{SnO}_{2}$, for the front contact. One goal is to minimize the product of the layer's electrical resistance and optical absorption, and basic work on the structure and properties of such oxides should be helpful. Perhaps more important, however, is the impact of the front contact, which is the first deposited layer in Figure 1, on the rest of the cell. Variations in its morphology should be added to the list of parameters that are systematically correlated with cell performance.

\section{Back Contact}

The back contact for a CdTe cell, as suggested above, has been another of the major challenges, since the goal is a low-resistance contact to a relatively wide band gap p-type material. Most of the successful solutions involve copper to form a thin $\mathrm{p}^{++}$or metallicalloy layer on the surface of the CdTe, followed by a thicker conducting layer, such as the carbon shown in Figure 1. The apparent problem is double-edged in that copper tends to diffuse away from the contact, leaving it more resistive, and towards the primary diode junction, decreasing its effectiveness. This thesis, however, is somewhat speculative, so better data on the actual profile of copper, and how it varies with CdTe deposition and contact formation techniques, as well as a theoretical model incorporating the effects of field-aided diffusion are needed. A different tack, which is already underway in earnest, is to find a copper-free contact strategy. The logical candidates are other p-dopants for $\mathrm{CdTe}$, such as $\mathrm{Hg}, \mathrm{Ag}$, and $\mathrm{Sb}$. $\mathrm{Sb}_{2} \mathrm{Te}_{3}$ shows good promise, ${ }^{28}$ but it is too early to declare the problem solved.

\section{ACKNOWLEDGEMENTS}

The authors are indebted to a large number of colleagues at both our own institutions and at the National Renewable Energy Laboratory (NREL), First Solar, Inc., ITN Energy Systems, and the Colorado School of Mines. We especially thank Peter Meyers of ITN for the use of Figure 3. Financial support was provided in part by NREL.

\section{REFERENCES}

1. J. Britt \& C. Ferekides, Appl. Phys. Lett. 62, 2851 (1993).

2. H. Ohyama,T. Aramoto, S. Kumazawa, H. Higuchi, T. Arita, S. Shibutani, T. Hishio, J. Hakajima, M. Tsuji, A. Hanafusa, T. Hibina, K. Omura, \& M. Murozono, $26^{\text {th }}$ Photovoltaics Specialists Conference-1997, pp. 343. 
3. H.S. Ullal, K. Zweibel, \& B. von Roedern, $26^{\text {th }}$ Photovoltaic Specialists Conference-1997, pp. 301.

4. A.M. Hermann, Solar Energy Materials and Solar Cells, 55, 75 (1998)

5. W. Fuhs and R. Klenk, Proc. $2^{\text {nd }}$ World Conf. on PV Solar Energy Conversion, 1998, pp. 381-386.

6. R. W. Birkmire and E. Eser, Ann. Rev. Mater. Sci., 27, 625-53 (1997).

7. P. V. Meyers and R. W. Birkmire, Prog. In Photovoltaics: Res. and Appl., 3, 393-402 (1995).

8. C. S. Ferekides, J. Britt, Y. Ma and L. Killian, Proc. 22nd IEEE Photovoltaic Specialists Conference, 1993, pp. 389-393.

9. C. S. Ferekides, K. Dugan, V. Ceekala, J. Killian, D. Oman, R. Swaminathan and D. L. Morel, Proc. 1st World Conference on Photovoltaic Energy Conversion, Hawaii, 1994, pp. 99-102.

10. B. E. McCandless and S. S. Hegedus, Proc. 21st IEEE Photovoltaic Specialists Conference, 1991, pp. 967972.

11. B. Depuydt, I. Clemminck, M. Burgelman and M. Casteleyn, Proc. 12th European Photovoltaic Solar Energy Conference, 1994, pp. 1554-1556.

12. P. V. Meyers, C. H. Liu and M. Doty, 'Method of making photovoltaic cells with chloride dip', US Patent 4,873,198 (1989).

13. T. X. Zhou, N. Reiter, R. C..Powell, R. Sasala and P. V. Meyers, Proc. lst World Conference on Photovoltaic Energy Conversion, Hawaii, 1994, pp. 103-106.

14. B. M. Basol, E. S. Tseng and D. S. Lo, 'Electrodeposition of thin-film heterojunction photovoltaic devices that utilize Cd rich $\mathrm{Hg}, \mathrm{Cd}$, Te', US Patent 4,548,681, (1984).

15. N. Nakayama, H. Matsumoto, K. Yamaguchi, S. Ikegami and Y. Hioki, Jpn. J. Appl. Phys., 15, 2281 (1976).

16. M.E. Özsan, D.R. Johnson, D.W. Lane, and K.D. Rogers, $12^{\text {th }}$ European Photovoltaic Solar Energy Conference-Proceedings, p. 1600 (1994).

17. K. Ohata, J. Saraie and T. Tanaka, Jpn. J. Appl. Phys., 12, 1198-1204 (1973); S.Y. Nunoue, T. Hemmi, and E. Kato, J. Electrochem. Soc. 137, (1990).

18. D.G. Jensen, B.E. McCandless, \& R.W. Birkmire, $25^{\text {th }}$ Photovoltaics Specialists Conference-1996, p. 773; R. B. Birkmire, J. E. Phillips, W. N. Shafarman, S. S. Hegedus, B. E. McCandless and T. A. Yokimcus, Polycrystalline thin-film materials and devices, Final Report to NREL, Subcontract No. XN-0-10023-1, NREL, Golden, CO, 1993.

19. A. L. Fahrenbruch, Sol. Cells, 21, 399-412 (1987).

20. J. P. Ponpon, Solid State Electron., 28(7), 689-706 (1985).

21. H. Uda, S. Ikegami and H. Sonomura, Sol. Energy Mater., 35, 293-298 (1994).

22. L. M. Fras, J. Appl. Phys., 49(2), 871-875 (1978).

23. B. E. McCandless, Y. Qu and R. W. Birkmire, Proc. lst World Conference on Photovoltaic Energy Conversion, Hawaii, 1994, pp. 107-110.

24. Y. S. Tyan, 'Semiconductor devices having improved low-resistance contacts to ptype CdTe and method of preparation', US Patent 4,319,069 (1980).

25. T. L. Chu, S. S. Chu, K. D. Han and M. Mantravadi, Proc. 20th IEEE Photovoltaic Specialists Conference, 1988, pp.1422-1425.

26. Landoldt-Bornstein Numerical Data and Functional Relationships in Science and Technology, vol 17b, "Physics of II-VI and I-VII Compounds, Semimagnetic Semiconductors," ed. by O. Madelung, M. Schulz, and H. Weiss (1982).

27. A.L. Fahrenbruch, $15^{\text {th }}$ NCPV Photovoltaics Program Review, p. 48 (ed. by M. Al-Jassim, J.P. Thornton, and J.M. Gee, AIP CP462, 1998) 
28. N. Romeo, A. Bosio, R. Tedeschi, and V. Canevari, $2^{\text {nd }}$ World Conf. and Exhibition on Photovoltaic Solar energy Conversion--Proceedings, p. 446 (1998) ed. by J. Schmid, et al.

29. R.C. Powell, U. Jayamaha, G.L. Dorer, \& H. McMaster, $15^{\text {th }}$ NCPV Photovoltaics Program Review, (ed. by M. Al-Jassim, J.P. Thornton, and J.M. Gee, AIP CP462, 1998) p. 31.

30. S.P. Albright \& J. Kester, "Module and Process Optimization and Device Efficiency Improvement for Stable. Low Cost, Large-area, CdTe-based, Photovoltaic Module Production," Final Technical Progress Report-1996. (Available from NTIS.)

31. X. Wu, P. Sheldon, Y. Mayathongdy, R.Ribelin, A. Mason, H.R. Moutinho, and T.J. Coutts, $15^{\text {th }}$ NCPV Photovoltaics Program Review, (ed. by M. Al-Jassim, J.P. Thornton, and J.M. Gee, AIP CP462, 1998) p. 37.

32. P.V. Meyers, National CdTe R\&D Team Meeting, May 1999, (unpublished).

33. A.D. Compaan, Z. Feng, G. Contreras-Puente, C. Narayanswamy, and A. Fischer, Mat. Res. Soc. Symp. Proc. 426, p. 367 (1996)

34. D. Grecu \& A.D. Compaan, (to appear in Appl. Phys. Lett. 1999); D. Grecu and A.D. Compaan, $15^{\text {th }}$ NCPV Photovoltaics Program Review, (ed. by M. Al-Jassim, J.P. Thornton, and J.M. Gee, AIP CP462, 1998) p. 224.

35. D. Grecu and A.D. Compaan, National CdTe R\&D Team Meeting, May 1999, (unpublished).

36. E.D. Jones, N.M. Stewart, J.B. Mullin, J. Cryst. Growth, 117, 244 (1992).

37. I. Lyubomirsky, M.K. Rabinal, D. Cahen, J. Appl. Phys. 81, 6684 (1997).

38. P.Y. Yu \& M. Cardona, Fundamentals of Semiconductors, $2^{\text {nd }}$ ed, (Springer-Verlag, Berlin, 1999) pp. $344 \mathrm{ff}$.

37. S.B. Zhang, S.-H. Wei, and A. Zunger, Phys. Rev. Lett. 78, 4059 (1997).

40. F.A. Selim and F.A. Kroeger, J. Electrochem. Soc., 124, 401 (1977).

41. I.L. Eisgruber, J.E. Granata, J.R. Sites, J. Hou, J. Kessler, Solar Energy Materials and Solar Cells, 53, 367 (1998).

42. D.H. Levi, L.M. Woods, D.S. Albin, \& T.A. Gessert, $15^{\text {th }}$ NCPV Photovoltaics Program Review, (ed. by M. Al-Jassim, J.P. Thornton, and J.M. Gee, AIP CP462, 1998) p. 461.

43. S. Smith, H.M. Cheong, B.D. Fluegel, J.F. Geisz, J.M. Olson, R. Dhere, L.L. Kazmerski, and A. Mascarenhas, $15^{\text {th }}$ NCPV Photovoltaics Program Review, (ed. by M. Al-Jassim, J.P. Thornton, and J.M. Gee, AIP CP462, 1998) p. 467.

44. See, for example, AMPS-1D, a one-dimensional solar-cell simulation program written under the direction of Prof. S. Fonash at Pennsylvania St. Univ. 


\title{
NEXT GENERATION THIN FILMS FOR PHOTOVOLTAICS: InGaAsN
}

\author{
E. D. Jones ${ }^{\mathrm{a}}$, A. A. Allerman ${ }^{\mathrm{a}}$, D. J. Friedman ${ }^{\mathrm{b}}$, J. F. Geisz ${ }^{\mathrm{b}}$, J. F. Klem ${ }^{\mathrm{a}}$, \\ Steven R. Kurtz ${ }^{a}$, N. R. Modine ${ }^{a}$, W. Shan ${ }^{c}$, C. Tu ${ }^{d}$, and W. Walukiewicz ${ }^{c}$ \\ a) Sandia National Laboratories, Albuquerque, NM \\ b) National Energy Renewable Laboratory, Golden CO \\ c) Lawrence Berkeley National Laboratories, Berkeley, CA \\ d) University of California San Diego, La Jolla, CA
}

\begin{abstract}
A new semiconductor alloy system, InGaAsN, has been identified as a candidate material for multi-junction solar cells having efficiencies greater than $40 \%$. The introduction of small amounts of nitrogen $(\sim 2 \%)$ into the InGaAs alloy system greatly reduces the band gap energy, with reductions approaching $0.4 \mathrm{eV}$ for $2 \%$ nitrogen content! With the appropriate ratio of indium to nitrogen concentrations, InGaAsN can be lattice matched to GaAs.
\end{abstract}

\section{INTRODUCTION}

The quaternary alloy system, InGaAsN, is a new material system that appears to have many exciting and important device applications. Because of a large negative bowing parameter, ${ }^{1,2}$ the addition of small amount of nitrogen to the $1.4 \mathrm{eV}$ bandgap energy GaAs system lowers the energy! In addition to lowering the bandgap energy, adding indium to GaAsN strain compensates the effect of nitrogen, resulting in a material system with bandgap energies $\sim 1 \mathrm{eV}$ and lattice matched to GaAs! The InGaAsN, alloy system has been identified as a key candidate material for long wavelength laser systems ${ }^{3-5}$ and high-efficiency multi-junction solar cells. ${ }^{6,7}$ Before the successful realization of multijunction solar cells which utilize thin films of InGaAsN as an integral part, questions regarding the role of the nitrogen isoelectronic atom in GaAs must be addressed. In particular: (1) What is the physical (or chemical) origin of the large bandgap reduction? (2) Are the nitrogen induced states extended (band-like) or localized (impurity-like)? (3) Are the observed low conduction-band mobilities intrinsic to InGaAsN? and (4) Are recombination times between the conduction and valence band states seriously affected?

This paper will address the aforementioned questions by reviewing some of the current experimental and theoretical information for the InGaAsN material system. Section 2 discusses InGaAsN thin film metalorganic chemical vapor deposition (MOCVD) and molecular beam epitaxy (MBE) growth techniques and growth parameters which result in the highest quality films suitable for incorporating into multijunction solar cells. The topic of Section 3 is the measurement minority carrier diffusion lengths. Also discussed in Section 3 are measurements of carrier lifetimes and internal quantum efficiencies. Photomodulated absorption spectroscopy results are discussed in Sec. 4 and an analysis of the data in terms of a simple two level system with anticrossing behavior is shown to give good agreement. Results from first principle band structure calculations using the local density approximation are presented in Sec. 5. Here it is shown that while the calculated value for the bandgap energy is in poor agreement with experiment, the agreement between the experimental and theoretical bandgap energy pressure coefficients is excellent. Section 6 outlines some 
key issues that need to be solved before InGaAsN can assume its anticipated role as the next generation thin film material for high efficiency solar cells. Finally, conclusions are presented.

\section{MOCVD Growth}

\section{THIN FILM GROWTH}

Thin $(<0.5 \mu \mathrm{m}) \mathrm{In}_{\mathrm{x}} \mathrm{Ga}_{1-\mathrm{x}} \mathrm{As}_{1-\mathrm{y}} \mathrm{N}_{\mathrm{y}}$ films were grown using trimethylindium (TMIn), trimethylgallium (TMG), 100\% arsine and dimethylhydrazine (DMHy). Dimethylhydrazine was used as the nitrogen source since it has a lower disassociation temperature than ammonia and has a vapor pressure of approximately 110 torr at $18^{\circ} \mathrm{C}$. It was found that unintentionally doped InGaAsN epilayers are p-type. InGaAsN films for Hall and optical measurements were grown on semi-insulating GaAs orientated $2^{\circ}$ off (100) towards $<110>$. Lattice matched films $\left(\delta \mathrm{a} / \mathrm{a}<8 \times 10^{-4}\right.$ )were grown at $600^{\circ} \mathrm{C}$ and 60 torr using a V/ III ratio of 97 , a $\mathrm{DMHy} / \mathrm{V}$ ratio of 0.97 and a $\mathrm{TMIn} / \mathrm{III}$ ratio of 0.12 . The growth rate was $10 \AA / s$. These conditions resulted in films with an indium mole fraction of $0.07 \pm 0.005$ and a nitrogen mole fraction of $0.022 \pm 0.003$. The composition was determined by calibration growths of GaAsN and InGaAs along with double crystal x-ray diffraction measurements. The nitrogen composition of bulk films was also confirmed from elastic recoil detection measurements. A significant increase in photoluminescence intensity was observed from these films following a post-growth anneal. Ex-situ, post-growth anneals were carried out in a rapid thermal anneal system under nitrogen using a sacrificial $\mathrm{GaAs}$ wafer in close proximity to the InGaAsN sample.Undoped InGaAsN/GaAs strained quantum wells are also of great interest to $1.3 \mathrm{~mm}$ vertical-cavity surface-emitting lasers. Incorporation of $\mathrm{N}$ in GaInAs, however, is found to quench photoluminescence (PL), but rapid thermal annealing (RTA) can improve the PL intensity. ${ }^{8}$ For solar cell applications a $1-2 \mu \mathrm{m}$ thick layer, lattice-matched to the GaAs substrate, with n- and p-type doping is desired.

Nitrogen incorporation on the order of $1 \%$ into GaAs has also been accomplished using plasma-cracked $\mathrm{NH} 3,{ }^{9}$ dimethylhydrazine (DMHy) ${ }^{10}$ and hydrazine as $\mathrm{N}$ sources. By far, the most common nitrogen source currently is dimethylhydrazine due to its easy incorporation into existing MOCVD systems and high vapor pressure.

Typical MOCVD growth of epitaxial $\operatorname{In}_{\mathrm{x}} \mathrm{Ga}_{1-\mathrm{x}} \mathrm{As}_{1-\mathrm{y}} \mathrm{N}_{\mathrm{y}}$ layers on GaAs use trimethylindium (TMIn), trimethylgallium (TMG), arsine and dimethylhydrazine (DMHy) as sources. Lattice matched films $(\delta \mathrm{a} / \mathrm{a}<8 \times 10-4)$ can be grown at $600^{\circ} \mathrm{C}, 60$ torr and $10 \AA$ $\mathrm{s}$ using a V/III ratio of 97 , a DMHy/V ratio of 0.97 and a TMIn/III ratio of 0.12 . These conditions result in films with an indium mole fraction of $0.07 \pm 0.005$ and a nitrogen mole fraction of $0.022 \pm 0.003$. The composition has been determined by calibration growths of GaAsN and InGaAs along with double crystal x-ray diffraction measurements. The nitrogen composition of bulk films has also been confirmed from elastic recoil detection measurements and secondary ion mass spectroscopy (SIMS). A wide range of other growth conditions have also resulted in lattice-matched InGaAsN films, but the material must be grown at low temperatures and high $\mathrm{DMH} / \mathrm{V}$ ratios ${ }^{11}$ to incorporate sufficient nitrogen. Nitrogen incorporation also appears to be suppressed with increasing In content. $^{12}$

Current challenges in the growth of InGaAsN by MOCVD techniques include high background carrier concentrations, unintentional incorporation of impurities, intentional doping, and low diffusion lengths. The most important issue for solar cell devices is 
(a)

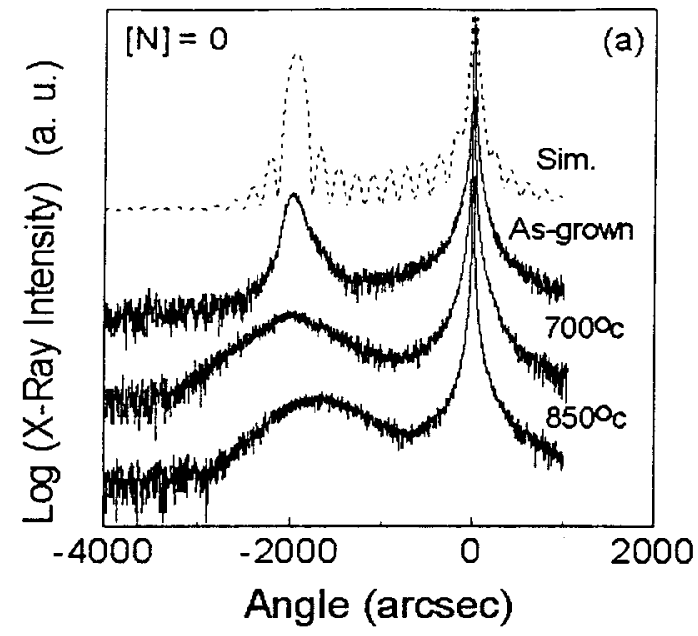

(b)

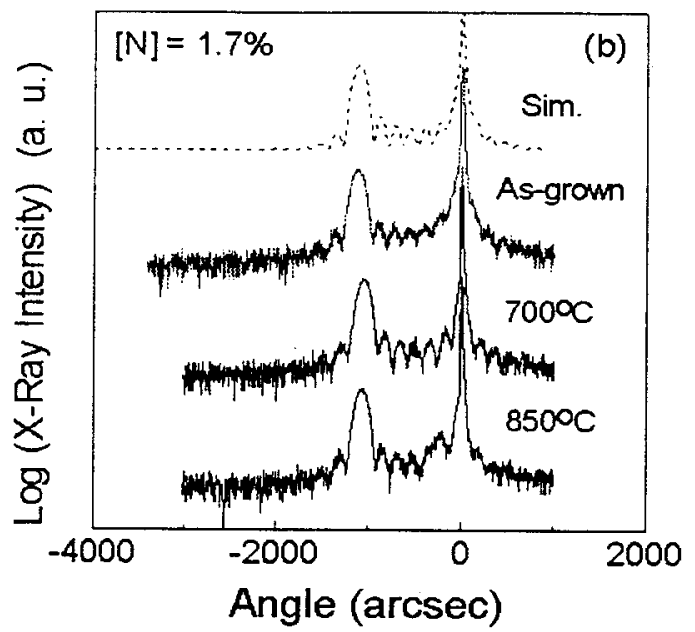

Figure1. X-ray rocking curves of as-grown and annealed $\mathrm{In}_{0.108} \mathrm{Ga}_{0.892} \mathrm{As}$ (Fig. 1(a)) and $\mathrm{In}_{0.108} \mathrm{Ga}_{0.892} \mathrm{~N}_{0.017} \mathrm{As}_{0.983}$ (Fig. 1(b)) samples, respectively. The dashed lines correspond to simulation results.

minority carrier diffusion lengths. Growth of InGaAsN to date has not yielded any material with sufficiently long diffusion lengths for typical high efficiency III-V structures. ${ }^{12}$ The reason for these low diffusion lengths remains unclear. Recent understanding of the band structure of this material explains observed low electron mobilities, but this alone does not completely explain the poor diffusion lengths. Theoretical calculations based on the current understanding of the band structure has not yet been able to predict the intrinsic limits to carrier lifetimes in this material. Direct, reliable measurements of minority carrier lifetimes and diffusion lengths also remain elusive. These topics are discussed in greater detail in Section 3.

Annealing of the material has been shown to improve PL intensities, ${ }^{8}$ hole mobilities, ${ }^{13}$ and cell characteristics. ${ }^{14}$ Reduced deep-level densities by annealing have also been reported. ${ }^{15}$ Carrier concentration can also be dramatically affected by annealing. ${ }^{13}$ Annealing conditions (e.g. ambient gas, temperature, time) can dramatically affect the results of annealing but these effects are not well understood because the actual microscopic effect of annealing the material is not yet known.

InGaAsN grown by MOCVD on GaAs is typically found to be p-type $\sim 1 \times 10^{17} \mathrm{~cm}^{-3}$. High carbon and hydrogen contamination in MOCVD-grown InGaAsN has been observed by SIMS. It has been postulated by many that the p-type background is due to the carbon acceptor partially passivated by hydrogen, but this has not been proven. The role of these unintentional contaminants in terms of potential deep trap centers that reduce diffusion lengths has not been deciphered. Demonstration of hydrogen and carbon free material may help elucidate this question. Intentional p-type doping with $\mathrm{C}$ and $\mathrm{Zn}$ has proved to be straightforward, but $n$-type doping remains problematic. The efficiency of Si doping efficiency by disilane is greatly reduced in InGaAsN materials in a temperature dependant manner implying problems with pre-reaction in the gas-phase. Se doping with $\mathrm{H}_{2} \mathrm{Se}$ on the other hand is extremely efficient at the low temperature, low arsine pressures typically employed. 


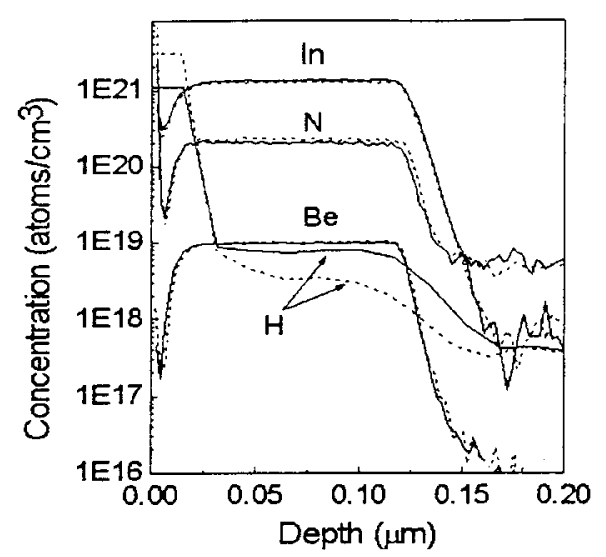

Figure 2. SIMS profiles of as-grown (solid lines) and $700^{\circ} \mathrm{C}$-annealed (dashed lines) for the $\mathrm{Ga}_{0.892} \mathrm{In}_{0.108} \mathrm{~N}_{0.017} \mathrm{As}_{0.983}$ samples.

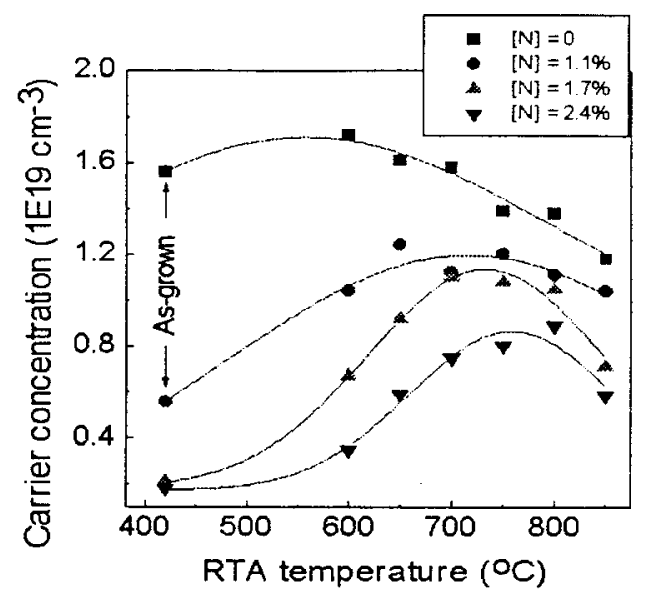

Figure 3. Free carrier concentrations as a function of RTA temperature for asgrown and annealed InGaAsN samples

\section{MBE Growth}

Highly Be-doped p-type $\left(\sim 1 \times 10^{19} \mathrm{~cm}^{-3}\right), 110$-nm-thick $\operatorname{In}_{0.108} \mathrm{Ga}_{0.892} \mathrm{~N}_{\mathrm{X}} \mathrm{As}_{1-\mathrm{x}}$ layers on semi-insulating GaAs substrates have been grown ${ }^{16}$ by gas-source molecular beam epitaxy with cracked arsine and a RF plasma nitrogen radical beam source. The compositions were determined by X-ray rocking curve (XRC) measurements and simulations based on the dynamical theory. A 10 second RTA was performed on these samples with halogen lamps in a flowing $\mathrm{N}_{2}$ ambient. During RTA, the samples were put on a GaAs wafer faceto-face to prevent the loss of arsenic at elevated temperatures.

Figures $1 \mathrm{a}$ and $1 \mathrm{~b}$ show XRCs of as-grown and annealed $\mathrm{In}_{0.108} \mathrm{Ga}_{0.892} \mathrm{As}$ and $\mathrm{In}_{0.108} \mathrm{Ga}_{0.892} \mathrm{~N}_{0.017} \mathrm{As}_{0.983}$ samples, respectively. The dashed lines correspond to simulation results. The $\mathrm{In}_{0.108} \mathrm{Ga}_{0.892} \mathrm{~N}_{0.017} \mathrm{As}_{0.983}$ peak shifts closer to the GaAs substrate peak, demonstrating that adding $\mathrm{N}$ into $\mathrm{In}_{0.108} \mathrm{Ga}_{0.892} \mathrm{As}$ layer does reduce the net compressive strain of the system. For N-containing samples, there are clear Pendelloesung fringes, indicating high crystalline quality and uniformity of the film, and the smooth interfaces between GaAs and InGaAsN. After RTA $\left(700^{\circ} \mathrm{C}\right.$ for $\left.10 \mathrm{~s}\right)$, the $\operatorname{In}_{0.108} \mathrm{Ga}_{0.892} \mathrm{As}$ peak broadens, indicating strain relaxation. For $\mathrm{In}_{0.108} \mathrm{Ga}_{0.892} \mathrm{~N}_{0.017} \mathrm{As}_{0.983}$, however, the Pendelloesung fringes after RTA at $700^{\circ} \mathrm{C}$ are sharper than those of the as-grown sample, indicating improved structural quality. After annealing at $850^{\circ} \mathrm{C}$, the XRC peaks are still well resolved, indicating the $\operatorname{In}_{0.108} \mathrm{Ga}_{0.892} \mathrm{~N}_{0.017} \mathrm{As}_{0.983}$ layer remains pseudomorphically strained and has better thermal stability than $\operatorname{In}_{0.108} \mathrm{Ga}_{0.892}$ As due to reduced lattice-mismatch $(0.37$ vs. $0.7 \%)$.

Secondary ion emission spectroscopy was performed on the samples to check the Be diffusion after RTA. It is found that no $\mathrm{H}$ is incorporated in $\mathrm{In}_{0.108} \mathrm{Ga}_{0.892} \mathrm{As}$, but $\mathrm{H}$ is incorporated alongside $\mathrm{N}$ in as-grown InGaAsN samples, possibly due to the very large electronegativity of $\mathrm{N}$ atoms ( $\mathrm{N}: 3.0$, As: 1.57). The higher the $\mathrm{N}$ concentration is, the 
more the $\mathrm{H}$ concentration. The free carrier concentration decreases with $\mathrm{N}$ incorporation mainly due to $\mathrm{H}$ passivation, which is also reported in MOCVD-grown InGaAsN samples. Figure 2 shows the SIMS profiles of as-grown (solid lines) and $700^{\circ} \mathrm{C}$-annealed (dashed lines) for $\operatorname{In}_{0.108} \mathrm{Ga}_{0.892} \mathrm{~N}_{0.017} \mathrm{As}_{0.983}$ samples. By annealing, $\mathrm{H}$ atoms dissociate from the $\mathrm{In}_{0.108} \mathrm{Ga}_{0.892} \mathrm{~N}_{0.017} \mathrm{As}_{0.983}$ layer, so the $\mathrm{H}$ concentration is reduced. With increasing RTA temperature, the $\mathrm{H}$ concentration is further decreased, and the free carrier concentration increases. There is no detectable Be diffusion at $700^{\circ} \mathrm{C}$ RTA. At $850^{\circ} \mathrm{C}$, some diffusion could be detected, and the free carrier concentration is also decreased.

Figure 3 shows the free carrier concentrations as a function of RTA temperature for asgrown and annealed InGaAsN samples. With the $\mathrm{N}$ concentration increasing from 0 to 0.024 , the carrier concentration of the as-grown samples decreases by one order of magnitude, and the Hall mobility decreases from 60 to $30-45 \mathrm{~cm}^{2} / \mathrm{Vs}$. After RTA at $700^{\circ} \mathrm{C}$, the carrier concentration of $\mathrm{N}$-containing samples is increased to half that of GaInAs and the Hall mobility also increases to $\sim 50 \mathrm{~cm}^{2} / \mathrm{Vs}$. Therefore, the product of carrier concentration and hole mobility is increased to half that of the GaInAs sample.

Several n-type InGaAsN layers were also grown ${ }^{16}$, and the mobility is rather low, similar to MOCVD layers. For a carrier concentration of $10^{17}$ to $10^{18} \mathrm{~cm}^{-3}$ the room-temperature mobility is $\sim 200 \mathrm{~cm}^{2} / \mathrm{Vs}$, which can be explained by the large electron effective mass and significant alloy scattering. Performing RTA shows that hydrogen atoms not only passivate the $\mathrm{Si}$ dopants but also act as n-type dopants. In summary, $\mathrm{N}$ incorporation in InGaAsN results in lower strain, better structural quality and thermal properties. Since here, hydrogen can come from cracked arsine, we now compare to MBE growth results using a solid As source. ${ }^{17}$

For InGaAsN samples grown by MBE using a solid As source, the SIMS measurements detected no $\mathrm{H}$ in these samples above the instrumental background of approximately $1 \times 10^{18} \mathrm{~cm}^{-3}$. Similarly, the concentration of $C$ was below $1 \times 10^{16} \mathrm{~cm}^{-3}$. On the other hand, B was detected at levels between $5 \times 10^{16}$ and $3 \times 10^{17} \mathrm{~cm}^{-3}$, depending on the $\mathrm{N}$ plasma source and power level used. This most likely results from sputtering of the boron nitride plasma chamber in the RF plasma source. In addition, a slight but significant increase in the level of $\mathrm{O}$ was detected for InGaAsN as compared to GaAs.

Unintentionally doped samples were p-type with room-temperature carrier concentrations of approximately $1 \times 10^{16} \mathrm{~cm}^{-3}$, and mobilities of approximately $200 \mathrm{~cm}^{2} / \mathrm{Vs}$. After annealing at $700^{\circ} \mathrm{C}$ for 15 minutes, the hole concentration increased slightly, to $2 \times 10^{16}$ $\mathrm{cm}^{-3}$, while the mobility remained essentially unchanged. $\mathrm{N}$-type samples were also grown by doping with Si. Electron concentrations in these samples were factors of 10-20 lower than the estimated $\mathrm{Si}$ atomic concentrations of $5 \times 10^{17}$ to $2 \times 10^{18} \mathrm{~cm}^{-3}$, and the mobilities were $200-300 \mathrm{~cm}^{2} / \mathrm{Vs}$. Upon annealing, the electron concentrations increased by a factor of two, and the mobilities increased slightly.

\section{MINORITY CARRIER EFFECTS}

To date, $1.0 \mathrm{eV}$ bandgap InGaAsN has displayed promising minority carrier diffusion, required for solar cell operation, under very limited conditions. Under specialized growth and post -processing procedures, hole diffusion lengths as large as 0.6 to $0.8 \mu \mathrm{m}$ have been observed in n-type, $1.0 \mathrm{eV}$ InGaAsN, lattice matched to $\mathrm{GaAs}^{7}$. Negligible electron diffusion has been observed in p-type material. Further research is required to understand 


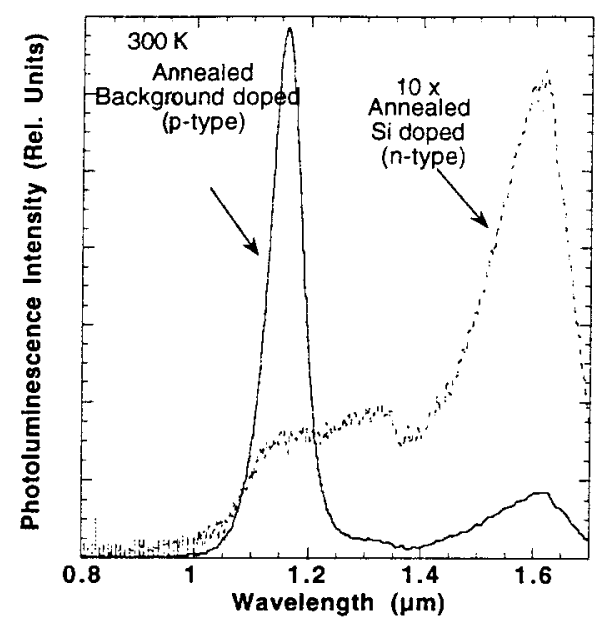

Figure 4. Photoluminescence spectra of $\mathrm{n}$ Figure 5. Time-resolved transmission of and p-type $\mathrm{In}_{0.07} \mathrm{Ga}_{0.93} \mathrm{As}_{0.98} \mathrm{~N}_{0.02}$, after annealed p-type $\mathrm{In}_{0.07} \mathrm{Ga}_{0.93} \mathrm{As}_{0.98} \mathrm{~N}_{0.02}$ at annealing.

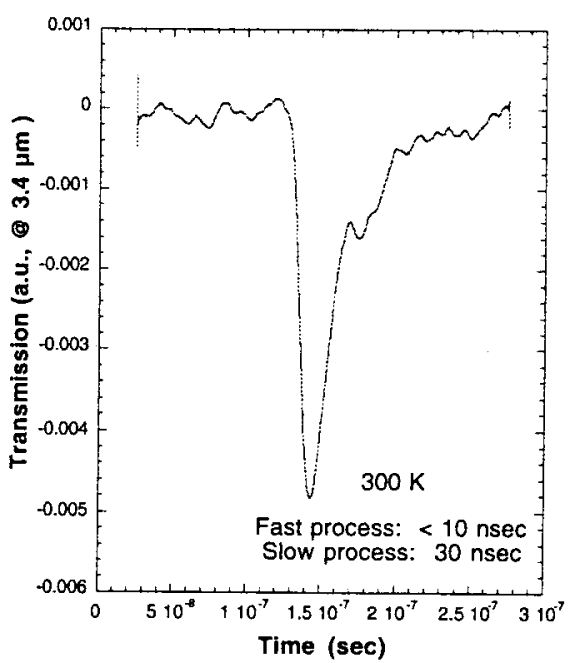

$3.4 \mu \mathrm{m}$, after pulsed excitation of electronhole pairs.

those properties of InGaAsN which limit minority carrier diffusion and to identify material growth and processing conditions which produce viable material for solar cells.

Optical $^{7}$ and DLTS ${ }^{15}$ studies show that InGaAsN (In=7\%, N=2\%) grown by MOCVD has several defects associated with doping and $\mathrm{N}$ incorporation. Photoluminescence spectra are shown in Fig. 4 for InGaAsN films grown under the same conditions and with nominally the same compositions as the $\mathrm{n}$ - and p-type regions of the solar cell. A band-edge photoluminescence peak was not observed in as-grown $\operatorname{In}_{0.07} \mathrm{Ga}_{0.93} \mathrm{As}_{0.98} \mathrm{~N}_{0.02}$ films at $300 \mathrm{~K}$. After an ex-situ annealing process, a band-edge photoluminescence peak, approximately $60 \mathrm{meV}$ linewidth, was observed in the p-type films. N-type doping with Si appears to introduce yet another defect or impurity into the InGaAsN. ${ }^{15}$ With Si doping, the bandedge photoluminescence was weak both before and after ex-situ annealing (Fig. 4).

Consistent with photoluminescence results, initial studies indicate that carrier lifetime is longest for annealed, p-type $\operatorname{In}_{0.07} \mathrm{Ga}_{0.93} \mathrm{As}_{0.98} \mathrm{~N}_{0.02}$ films. $\mathrm{N}$-type or as-grown films had significantly shorter free carrier lifetimes. The carrier lifetime in annealed p-type material is controlled by a fast $(<10 \mathrm{nsec})$ process and a slower, temperature dependent, defectmoderated process ( $50 \mathrm{nsec} @ 300 \mathrm{~K})$ and is shown in Fig. 5. This short lifetime $(\approx 5 \mathrm{nsec})$ and typical Hall mobilities observed in our samples $\left(\approx 200 \mathrm{~cm}^{2} / \mathrm{Vs}\right)$ predict carrier diffusion lengths of $\approx 1 \mu \mathrm{m}$. Alloy scattering rates for electrons should be greater for XAsN or $\mathrm{XSbN}$ systems than other III-V alloys, and for InGaAsN, electron mobility is limited by alloy scattering. Alloy scattering is dependent upon spatial fluctuations in the chemical potential for the electrons or holes. These energy fluctuations are very large for GaAsN, and the fluctuations are proportional to the differences in electronegativity between the binary constituents (GaAs vs. GaN for example) or alternatively, the variation of conduction band energy with composition 

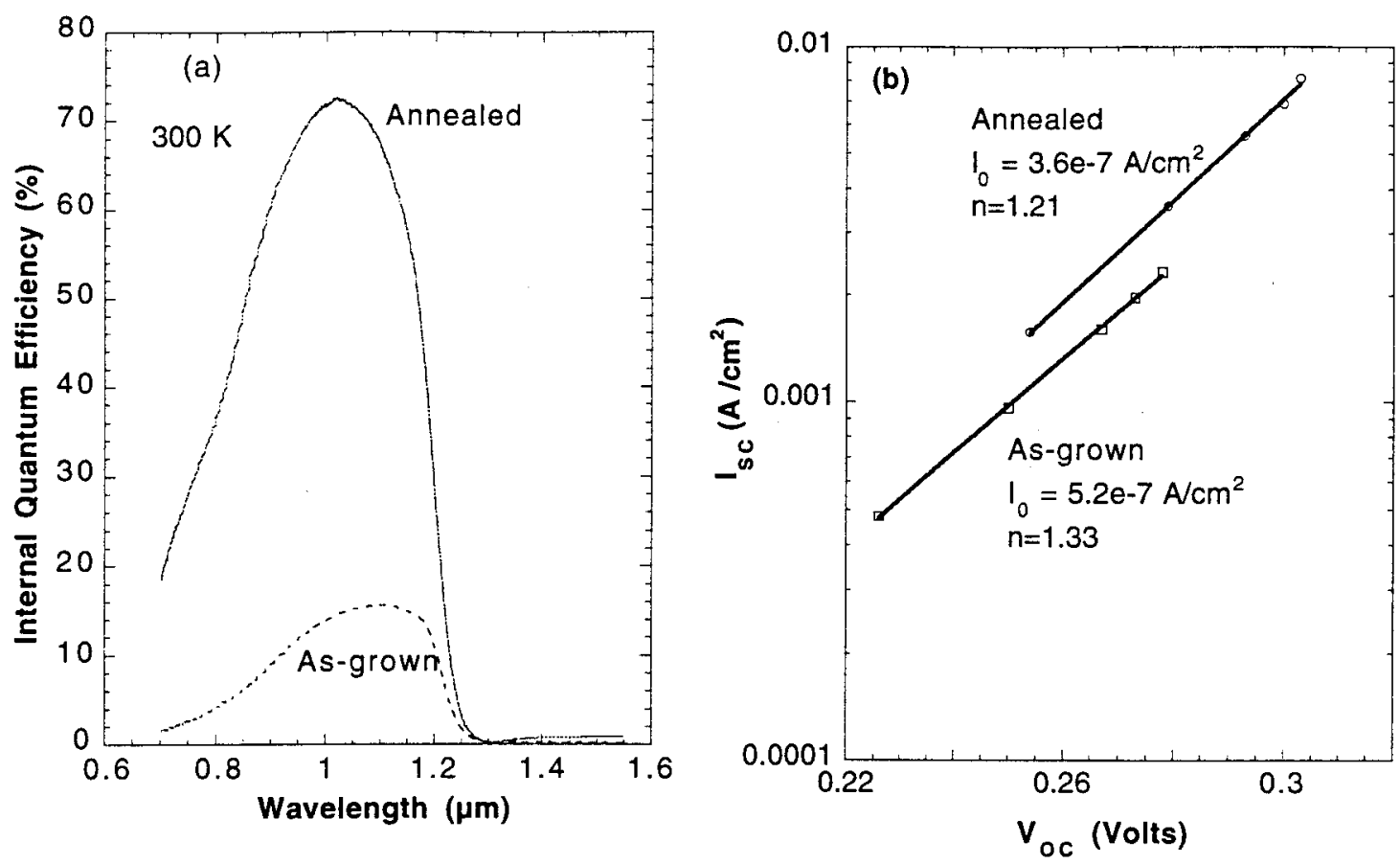

Figure 6. Internal quantum efficiencies of annealed and as-grown, $1.0 \mathrm{eV}$ InGaAsN solar cells (Fig. $6(\mathrm{a}))$ and short-circuit current $\left(\mathrm{I}_{\mathrm{sc}}\right)$ and open-circuit voltage $\left(\mathrm{V}_{\mathrm{oc}}\right)$ at different broadband light intensities for the cells in (Fig. 6 (a)) is shown in Fig. 6 (b)

Based on these annealing studies, a solar cell was constructed with a $1.0 \mu \mathrm{m}$ thick, $\mathrm{n}$ type $\left(2 \times 10^{17} \mathrm{~cm}^{-3}\right.$, Si doped $) \mathrm{In}_{0.07} \mathrm{Ga}_{0.93} \mathrm{As}_{0.98} \mathrm{~N}_{0.02}$ emitter grown on a $1.0 \mu \mathrm{m}$ thick, $\mathrm{p}$ type $\left(4 \times 10^{16} \mathrm{~cm}^{-3}\right.$, background doped) base. Internal quantum efficiency curves for both the annealed and as-grown cells are shown in Figure 6. The photoresponses extended out to the band-edge of the InGaAsN at $1.2 \mu \mathrm{m}$. Peak internal quantum efficiencies of $>70 \%$ were obtained for the annealed cell. Comparing the annealed and as-grown cells, annealing improved the quantum efficiency by roughly a factor of 5 . Comparing the performance of a thick n-type emitter solar cell with thin n-type emitter cells and other alternative designs, it is found that negligible electron diffusion is occurring in the $1.0 \mathrm{eV} \mathrm{p}$-type material (annealed or as-grown). To date, high quantum efficiencies have only been obtained with cell designs utilizing hole diffusion in n-type material. ${ }^{7}$

To estimate the minority carrier diffusion length in our cells, the photocurrent response versus bias was studied. Estimates show that the hole diffusion lengths are in the range of 0.6 to $0.8 \mu \mathrm{m}$ (annealed) and 0.2 to $0.3 \mu \mathrm{m}$ (as-grown). Despite the poor quality of the ntype material as indicated by optical studies, the improved efficiency of the annealed InGaAsN cell was due to diffusion of holes in the n-type emitter. ${ }^{7}$ Electron diffusion lengths remain negligible $(\approx 0.1 \mu \mathrm{m})$, leaving open the possibility that intrinsic, isovalent $\mathrm{N}$ sites produce electron localization in InGaAsN.

\section{PHOTOMODULATION SPECTROSCOPY}

Photomodulation spectroscopy has been used to measure the band gap energy of the $\mathrm{In}_{\mathrm{x}} \mathrm{Ga}_{1-\mathrm{x}} \mathrm{N}_{\mathrm{y}} \mathrm{As}_{1-\mathrm{y}}$ samples under hydrostatic pressure. The measurements were carried out 


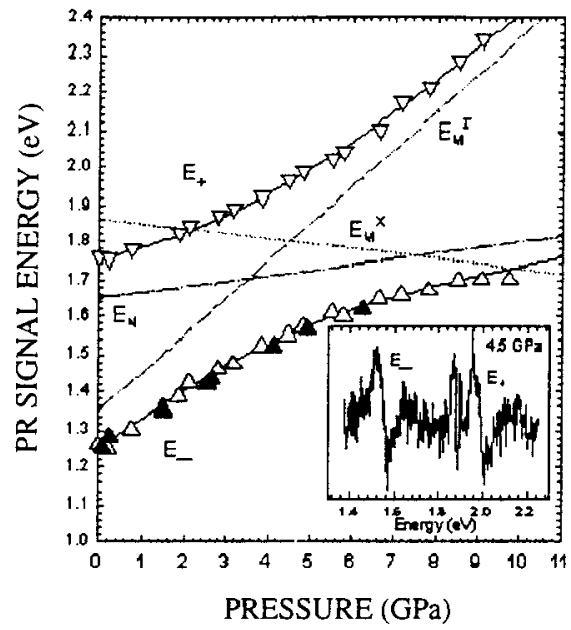

Figure 7. Change of the optical transition energies ( $\mathrm{E}_{-}$and $\mathrm{E}_{+}$) in $\mathrm{In}_{0.05} \mathrm{Ga}_{0.95} \mathrm{~N}_{0.012} \mathrm{As}_{0.988}$ as a function of applied pressure. The open triangles are experimental data. The solid lines are theoretical calculation results for the band anticrossing model. The dashed, dotted, and dot-dashed lines are the pressure dependence of the $\Gamma$ and $X$ conduction-band edges of the $\mathrm{In}_{0.05} \mathrm{Ga}_{0.95} \mathrm{As}$ matrix and the $\mathrm{N}$ level relative to the top of the valence band, respectively. The inset shows a photoreflectance (PR) spectrum taken at 4.5 GPa. The narrow $\mathrm{PR}$ spectral feature at energy below $\mathrm{E}_{+}$is from the GaAs substrate.

in either transmission or reflection geometry at room temperature. Photomodulation spectroscopy is a differential method probing the variation of the absorptivity of the samples caused by the modulation of physical parameters such as the built-in surface field in the system. ${ }^{18}$ The resulting spectra are characterized by features arising from direct interband transitions at high symmetry points in the band structure. Quasi-monochromatic light from a halogen tungsten lamp dispersed by a $0.5-\mathrm{m}$ monochromator was focused on the samples

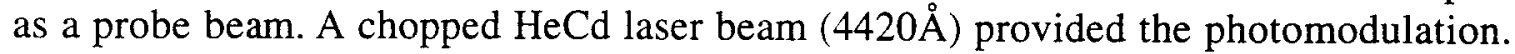
The photo-modulated transmission signals were detected by either $\mathrm{Ge}$ or Si photodiode using a phase-sensitive lock-in amplification system. Application of pressure was accomplished by mounting small sample chips with sizes of $\sim 200 \times 200 \mathrm{~mm}^{2}$ into gasketed diamond anvil cells.

It is found that in $\mathrm{N}$ containing material the fundamental gap or $\mathrm{E}_{0}$ transition splits into two transitions $E_{-}$that represents the lowest energy gap and a higher energy $E_{+}$transition. The splitting of the conduction band given by the energy difference $\Delta E=E_{+}-E_{\text {_ }}$ increases with increasing $N$ content. Also, as is shown in Fig. 7, the energies of the $E_{+}$and $E-$ transitions show characteristic dependence on the hydrostatic pressure. ${ }^{19}$ The observed effects find a simple and straightforward interpretation in terms of an anticrossing of a narrow band of localized $\mathrm{N}$ states and the extended states of the conduction band. The hydrostatic pressure changes the location of the $\mathrm{N}$-level relative to the conduction band leading to an exemplary case of a level anticrossing behavior. The band anticrossing model explains very well the pressure dependence of the $\mathrm{E}_{+}$and $\mathrm{E}_{\text {. }}$ transitions. It also accounts for the pressure-induced change in the relative intensities of the photomodulation spectra for these transitions. ${ }^{18}$

The interaction between localized $\mathrm{N}$-states and the conduction band leads also to a massive modification of the conduction band structure. Figure 8 shows the calculated dispersion relations for a parabolic conduction band interacting with dispersionless $\mathrm{N}$-band. The conduction band splits into two highly non-parabolic subbands. The curvatures, and thus also the density of states effective masses strongly depend on the energy and the relative location of the nitrogen energy level $\mathrm{E}_{\mathrm{N}}$ and the conduction band edge of the nitrogenfree host semiconductor matrix $\mathrm{E}_{\mathrm{M}}$. The predicted increase of the effective mass for the 


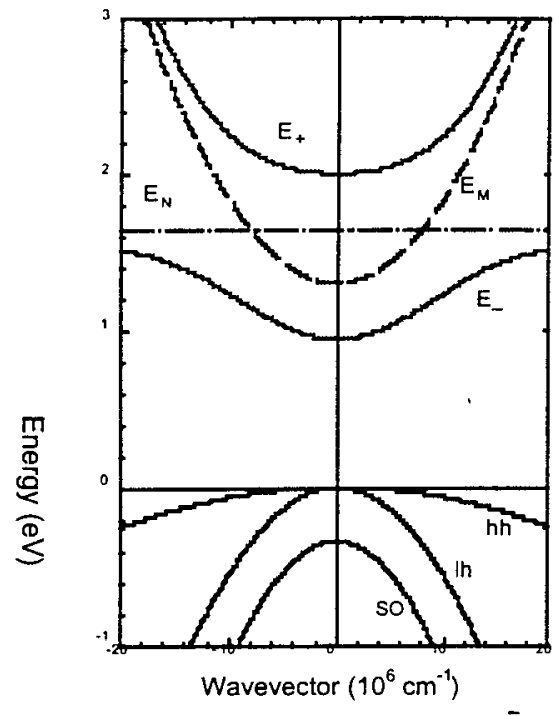

Figure 8. Calculated band structure showing anticrossing in the vicinity of the $\Gamma$ point in the Brillouin zone for GaAsN. The dashed line represents the unperturbed curvature of the $\Gamma$ conduction band of GaAs $\left(\mathrm{E}_{\mathrm{M}}\right)$ and the dot-dashed line is the energy location of the highly localized $\mathrm{N}$-state $\left(\mathrm{E}_{\mathrm{N}}\right)$. The anticrossing interaction between the extended conduction-band states and a narrow band of the $\mathrm{N}$ resonant states results in a splitting of the conduction band into two nonparabolic subbands $\left(E_{-}\right.$and $\left.E_{+}\right)$. The downward shift of the $E_{-}$ subband edge is responsible for the reduction of the fundamental band-gap energy commonly observed in III-N-V alloys.

lower subband is consistent with recent magnetoluminescence measurements on InGaAsN quantum wells. $^{20}$

The modifications of the conduction band structure have also profound effect on the transport properties of the conduction band electrons. It has been reported that the room temperature electron mobilities in InGaAsN alloys do not exceed $500 \mathrm{~cm}^{2} / \mathrm{Vs}$ which is at least one order of magnitude lower than electron mobilities observed in $\mathrm{N}$-free GaInAs alloys. This dramatic reduction of the electron mobilities can be explained by $\mathrm{N}$-related alloy disorder scattering. In a simple approximation the strength of the alloy scattering can be related to the $\mathrm{N}$-induced conduction band shift. In InGaAsN alloys this shift can be as large as $0.2 \mathrm{eV}$ per atomic percent of $\mathrm{N}$. This is at least an order of magnitude larger than typical band shifts observed in standard alloys. $\mathrm{N}$-induced enhancement of the conduction band effective mass is another factor leading to the electron mobility reduction. Model calculations show that for GaNAs with $2 \%$ of $\mathrm{N}$ and electron concentration of $1 \times 10^{17} \mathrm{~cm}^{-3}$ the mobility is reduced to $400 \mathrm{~cm}^{2} / \mathrm{Vs}$. This value is about 14 times smaller than the room temperature electron mobility in GaAs. ${ }^{21}$

The up-to-date research has demonstrated that group III-N-V alloys exhibit novel and quite extraordinary properties. However, future applications of these materials hinge on solving several outstanding issues associated with low electron mobilities and minority carrier lifetimes. Further research will be necessary to understand if the inferior material characteristics are intrinsic or extrinsic in nature. To these end systematic studies of factors affecting incorporation of lifetime limiting defects will be necessary. This will require a comprehensive approach that consists of a combination of materials synthesis and characterization techniques. Also more accurate calculations of the energy band structure and the effects of the modified conduction band on the electron mobility should be persuade to resolve the issue of intrinsic limitations of these materials. An important fundamental issue is the question whether III-N-V alloys is a unique system in which small change in 
alloy composition produces such a large change in properties or if there exist other system that exhibit similar behavior.

\section{BANDSTRUCTURE CALCULATIONS}

Both first-principles ${ }^{22-24}$ and empirical ${ }^{25-28}$ theoretical treatments for this material system have concentrated on understanding the dependence of the bandgap energy on nitrogen composition. Recently, Jones et. al. have reported ${ }^{29}$ the results of a first-principles electronic structure calculations based on the Kohn-Sham density functional theory with plane wave basis sets, ultrasoft pseudopotentials, and the local density approximation for the exchange-correlation functional. In construction of the pseudopotentials, the $\mathrm{Ga} 3 \mathrm{~d}$ electrons were treated as valence electrons in order to accurately represent any effects of a near resonance with the nitrogen $2 \mathrm{~s}$ level that has been observed in $\mathrm{GaN}^{30}$ The InGaAsN system in the experimentally relevant concentration range was modeled using a series of supercells of the zincblende GaAs structure each with a single arsenic replaced by a nitrogen. The lattice constants of the supercells were varied to simulate the effects of pressure, and for each cell, the ionic positions were relaxed using first-principles forces until the residual forces were less than $20 \mathrm{meV} / \AA$. In all cases, it was found ${ }^{29}$ that the nitrogen atom remained in the symmetric position during relaxation. In order to compare to experimental data, which is taken as a function of pressure, an $a b$ initio calculation of the system pressure was performed. Several supercells were investigated with the following stoichiometries: $\mathrm{Ga}_{32} \mathrm{As}_{31} \mathrm{~N}, \mathrm{Ga}_{64} \mathrm{As}_{63} \mathrm{~N}, \mathrm{Ga}_{108} \mathrm{As}_{107} \mathrm{~N}$, and $\mathrm{Ga}_{128} \mathrm{As}_{127} \mathrm{~N}$. These cells correspond to nitrogen concentrations of $3.13,1.56,0.93$ and $0.78 \%$, with the nitrogen atoms ordered in simple cubic, fcc, bcc, and simple cubic lattices respectively. The calculated band structures of the various supercells were qualitatively similar despite their differing symmetries, indicating that the nitrogen atoms interact weakly with each other at these low concentrations leading to the conclusion that the artificially ordered supercells provide an adequate model of the near-band-gap electronic structure of the disordered experimental system.

Figure 9 shows a representative band structure for the $3.13 \%$ system. It should be noted that the band structure is plotted with respect to the Brillouin zone of a 64-atom cell. Since the nitrogen substitution breaks the symmetry of the underlying zincblende structure, there is no uniquely defined way to "unfold" the band structure into the Brillouin zone of the primitive 2 -atom zincblende unit cell. The high symmetry points of the primitive GaAs cell fold into the $\Gamma$-point of the 64 -atom cell, and therefore in the presence of a real symmetry breaking term (such as produced by nitrogen substitution), one expects interactions between the resulting levels. The valence band and the conduction band are indicated by the heavy solid line. The conduction band is well separated from the other bands throughout most of the Brillouin zone, and it is quite dispersive with a bandwidth more than $1 \mathrm{eV}$. Likewise, the bands above the conduction band show a substantial amount of dispersion, and there is no evidence of a flat impurity-like band near the conduction band energies shown in Fig. 9. The absence of a nitrogen derived impurity-like state is supported by a decomposition of the wave-functions in terms of atomic-like orbitals, which shows that the conduction band has about $5 \%$ of its weight on the nitrogen atom, which is by far the highest fraction of any of the bands above the gap. ${ }^{22}$ The calculated bandgap is only $0.12 \mathrm{eV}$, while the experimental bandgap is of order $1 \mathrm{eV}$ for this concentration of nitrogen. This large error in the bandgap is a well known problem of the LDA. A central result of this calculation is that despite this large error in the absolute magnitude of 


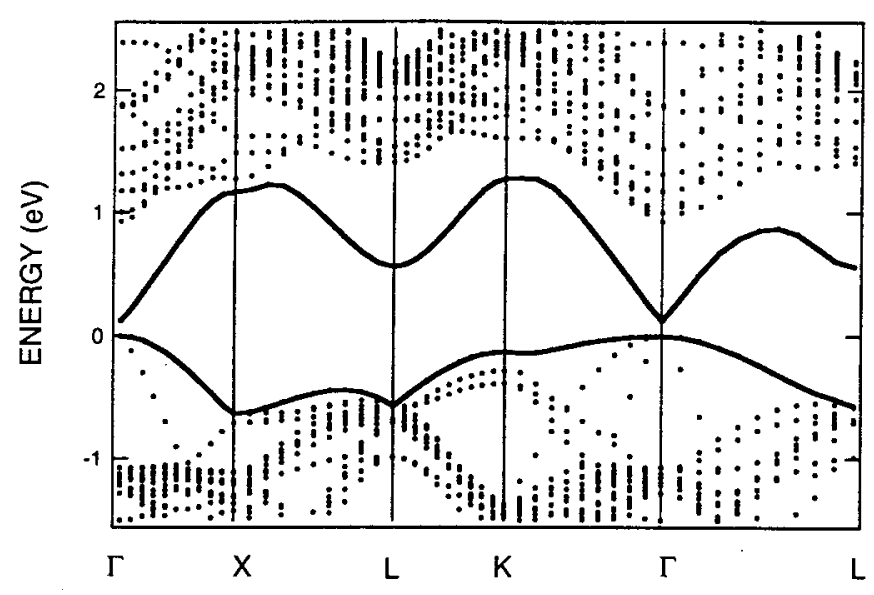

Figure 9. First principles local density approximation calculation for $3.3 \%$ nitrogen InGaAsN alloy. The solid lines, top and bottom, are respectively the conduction and valence bands.

the bandgap, the change in bandgap with lattice constant is in excellent agreement with experiment (see Fig.10).

A remarkable feature of Fig. 10 is the strongly non-linear dependence of the gap on the pressure. This is in marked contrast to the parent GaAs material and provides additional evidence, beyond the large reduction in the bandgap, that a few percent of nitrogen is producing remarkable changes in the material. In order to understand this nonlinearity, it is necessary to study additional bands above the conduction band. Figure 11 shows the theoretical pressure dependence of the $\Gamma$-point energies of several additional bands treated with the same analysis that was used for the conduction band in Fig. 10. Eight energy bands of the system are shown, but these fall into five degenerate groups. The figure shows three singlet states (filled circles), a doublet (open squares), and a triplet state (open triangles). Similar conclusions have also been recently reported by Mattila et. al. ${ }^{31}$

A common origin of nonlinear behavior of energy levels as a function of a parameter (such as pressure) is band repulsion. Band repulsion results from the mixing (hybridization) of bands in the same representation of the crystal symmetry group in such a way that level crossings are replaced with non-intersecting horizontal curves separated by a gaplike region. In Fig. 11, the highest singlet on the left and the triplet on the upper right bend downward due to repulsion from higher energy bands that have been omitted from the figure in order to improve its clarity.

With one exception, all of the bands within a few $\mathrm{eV}$ of the gap are observed to regain the degeneracies of pure GaAs to within of a few hundredths of an $\mathrm{eV}$ by the time the largest cell $(0.78 \% \mathrm{~N}$ concentration) is reached. The exception is the L-derived singlet, which remains split off from the triplet by about $0.1 \mathrm{eV}$. This suggests that this singlet may evolve into the impurity state observed at very low nitrogen concentrations. ${ }^{32,33}$ However, as mentioned above, this state does not act like an impurity state at the technologically interesting concentrations around $2 \%$. Furthermore, the L-derived singlet rises faster than the conduction band throughout the studied pressure range, and we do not see the upward curvature that would be expected if it was repelled by the conduction band. Therefore, the conclusion is that repulsion between the L-derived singlet and the conduction band con- 

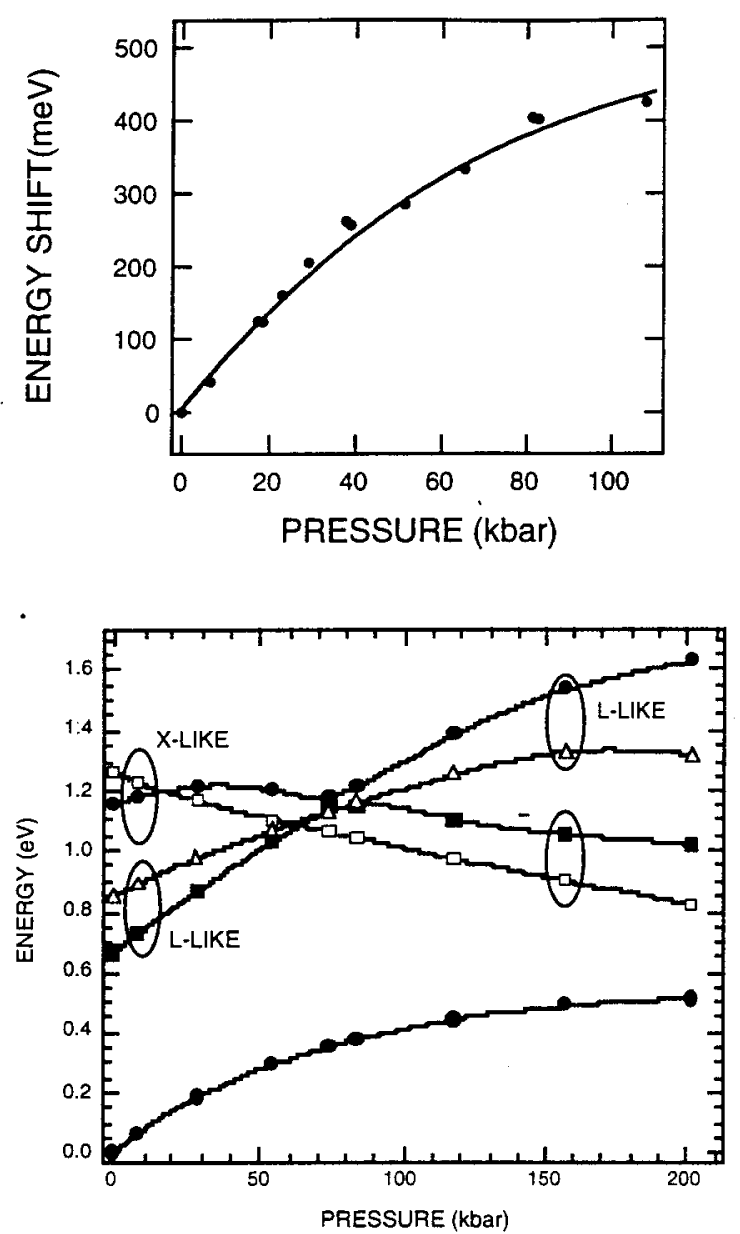

Figure 10. The pressure dependence of the 4$\mathrm{K}$ bandgap energy shift, as determined from by the PL-peak energy, is shown as solid circles. The solid curve drawn through the data is a results of a first principles LDA bandstructure calculation. ${ }^{28}$

Figure 11. Theoretical pressure dependence of the conduction-band states near the bandgap energy minimum for $2 \%$ nitrogen GaAsN alloy. The points are calculated and there are three singlets (filled circles), a doublet (open squares), and a triplet state (open triangles).

tributes at most a small amount to the nonlinearity of the conduction band. In contrast, Fig. 11 demonstrates almost textbook repulsion between the X-derived singlet and the conduction band at pressures over $100 \mathrm{kbar}$, and it is likely that this repulsion continues to lower pressures, even though the effect is obscured by the additional repulsion between the Xderived singlet and a higher state. Based on these observations, the authors proposed ${ }^{29}$ that repulsion from the $\mathrm{X}$-derived singlet is the chief cause of the experimentally observed nonlinear dependence of the bandgap on pressure. Based on the above, these calculations lead to the conclusion that the nonlinear dependence of the bandgap on pressure does not result from localized nitrogen states.

\section{InGaAsN Growth}

\section{ISSUES}

The growth of InGaAsN alloys by MOCVD is difficult due to the unusual sensitivity of the alloy to growth conditions and the possible interaction between Group III and Group V sources. The growth rate depends on the total group III flux when the group V transport is fixed as is typical of III- $V$ compound semiconductors. However, the growth rate has also been found to depend on the N/V ratio. Lower N/V ratios result in higher growth rates regardless of whether the $\mathrm{AsH}_{3}$ flow is increased or the DMHy flux is decreased. Alloy composition in also very sensitive to growth temperature and the $\mathrm{N} / \mathrm{V}$ gas phase ratio. The purity and chemical interaction of the nitrogen source (DMHy) now commonly used adds another uncertainty. Systematic investigation of how growth conditions 
influence material properties such as background impurity incorporation, carrier trap density and energies and minority carrier properties should be pursued.

\section{Localized vs. Delocalized States}

Continued basic research on $\mathrm{InGaAsN}$ is required to address problems concerning the true picture going from localized electron states to a delocalized concept. Detailed transport studies in compensated InGaAsN are required to determine if electron localization is intrinsic to InGaAsN. More defect studies (optical spectroscopy, DLTS, resonant Raman, etc.) are needed understand the atomic nature of the $\mathrm{N}$ site and clusters. Defect studies will also be critical to improving electron and hole diffusion and guiding material growth.

However, future applications of these materials hinge on solving several outstanding issues associated with low electron mobilities and minority carrier lifetimes. Further research will be necessary to understand if the material characteristics are intrinsic or extrinsic in nature. To these end systematic studies of factors affecting incorporation of lifetime limiting defects will be necessary. This will require a comprehensive approach that consists of a combination of materials synthesis and characterization techniques. Also more accurate calculations of the energy band structure and the effects of the modified conduction band on the electron mobility should be persuade to resolve the issue of intrinsic limitations of these materials. An important fundamental issue is the question whether III-N-V alloys is a unique system in which small change in alloy composition produces such a large change in properties or if there exist other system that exhibit similar behavior.

\section{Minority Carrier Lifetimes and Diffusion Lengths}

Continued basic research on InGaAsN is required to address problems identified in these initial studies. Detailed transport studies in compensated InGaAsN are required to determine if electron localization is intrinsic to InGaAsN. More defect studies (optical spectroscopy, DLTS, resonant Raman, etc.) are needed understand the atomic nature of the $\mathrm{N}$ site and clusters. Defect studies will also be critical to improving electron and hole diffusion and guiding material growth. Concurrent studies of prototype solar cells will be critical optimize solar cell design to accommodate deficiencies of the InGaAsN material.

\section{Solar Cell Design}

The requirement that solar cells have good minority-carrier properties (i.e. long diffusion lengths / lifetimes) to achieve high efficiencies sets constraints on the materials systems that can be used for epitaxial growth of multilayer cell structures. A key constraint for the various epilayers in the structure regards their lattice constants compared to that of the substrate: since interface strain due to lattice mismatch generally lead to extended structural defects which adversely affect the minority-carrier properties of the layer. While schemes have been devised to control the propagation of such defects, a lattice-matched system is significantly easier to work with, and is the approach discussed here. Note that the structural properties of an epilayer affect the structure of all layers grown upon it; therefore, lattice-matching of the third junction in a three- or four-junction stack will affect the minority-carrier properties not only of the third junction itself, but also of the other two junctions grown on top of the third junction. Thus the condition or property that $\mathrm{Ga}_{1-\mathrm{x}} \mathrm{In}_{\mathrm{x}} \mathrm{N}_{\mathrm{y}} \mathrm{As}_{1-\mathrm{y}}$ is lattice-matched to GaAs at $\mathrm{x}=3 \mathrm{y}$ is a key advantage of this alloy system. It should be noted, however, that lattice matching is not the only determinant of minority-carrier properties; as discussed below, the minority-carrier properties of (lattice- 
matched) InGaAsN are the key challenges in making a useful solar cell from this alloy system.

For a cell serving as the third junction under InGaP and $\mathrm{GaAs}$ junctions, the efficiency of the device depends sensitively on the band gap of the third junction. Assuming an ideal device, as the band gap is raised above its optimal value of $1 \mathrm{eV}$, the amount of light available between its band gap and the band gap of the GaAs cell above it decreases, decreasing the photocurrent which the third junction is capable of producing. In the seriesconnected two-terminal configuration discussed here, once the photocurrent of the third junction is reduced below that of the $\mathrm{InGaP} / \mathrm{GaAs}$ two-junction stack with which it is in series, the third junction will limit the photocurrent of the entire device, and will decrease rather than increase the overall device efficiency. On the other hand, as the band gap of the third junction is decreased below its $1 \mathrm{eV}$ optimum (the $1 \mathrm{eV}$ number assumes ideal device performance, in sharp contrast to the performance actually achieved for InGaAsN devices to date), the voltage of the third junction will decrease, reducing the efficiency boost provided by the third junction. Reducing the band gap of the third junction will also increase the photocurrent which the third junction is capable of producing; this may provide an approach to help meet the multijunction current-matching requirement for third junctions whose quantum efficiencies less than ideal. However, if a fourth junction is introduced below the three-junction InGaP/GaAs/InGaAsN structure for an additional efficiency boost, reducing the band gap of the third junction too far starts to steal too much light from the Ge fourth junction, once again reducing the overall multijunction device efficiency. The ability to control precisely the band gap of the third cell is therefore a requirement for the material used to fabricate it.

It should be emphasized that correctly measuring the IV curves for devices such as these, which are designed for operation under the spectral range of 1.0 to $1.4 \mathrm{eV}$, is not trivial. There are at least three causes for this difficulty: (1) the spectral range of 1-1.4 eV is very narrow, (2) the spectrum of the simulator's xenon lamp has multiple spikes in this spectral region, and (3) for terrestrial spectra, there are two large atmospheric-absorption notches in the 1.0-1.4 eV spectral range. Attempts to measure the current-voltage characteristics for these devices by using the conventional expedient of a reference cell are likely to result in significant errors if a xenon arc lamp is the light source.

Finally, concurrent studies of prototype solar cells will be critical optimize solar cell design to accommodate deficiencies of the InGaAsN material.

\section{CONCLUSIONS}

There has been considerable activity and progress during the 1998 to 1999 time period in obtaining new experimental data and thus allowing realistic theoretical modeling of the physical properties of the InGaAsN system. When all of the intrinsic properties are understood and extrinsic effects are identified, the real role or importance of InGaAsN as a candidate next generation thin film for photovoltaics material can be made.

\section{ACKNOWLEDGEMENTS}

The authors wish to thank Dr.'s R. M. Biefeld and A. Zunger for many valubale discussions and also for critical reading of the manuscript. Sandia is a multiprogram laboratory operated by Sandia Corporation, a Lockheed Martin Company, for the United States Department of Energy under contract DE-AC04-94AL85000. Part of this work was per- 
formed at Lawrence Berkeley Laboratories, supported by the Director, Office of Energy Research, Office of Basic Energy Sciences, Division of Materials Sciences, of the US Department of Energy under contract No. DE-AC03-76SF00098. The work at the National Energy Renewable Laboratories was supported by the U.S. Department of Energy under Contract No. DE-AC36-98-GO10337. The work at UCSD is supported in part by a UC MICRO program with Rockwell International and the U.S.Army Research Office under the MURI "Low Power and Low Noise Electronics Technologies for Wireless Communications".

\section{REFERENCES}

1. W. G. Bi and C. W. Tu, Appl. Phys. Lett. 70, 1608 (1997).

2. L. Malikova, F. H. Pollak, and R. Bhat, J. Electronic Materials 27, 484 (1998).

3. M. Kondow, K. Uomi, A. Niwa, T. Kitatani, S. Watahiki, and Y. Yazawa, Jpn. J. Appl. Phys. 35, 1273 (1996).

4. M. Kondow, T. Kitatani, S. Nakatsuka, M. C. Larson, K. Nakahara, Y. Yazawa, M. Okai, and K. Uomi, IEEE J. Selected Topics in Quantum Electronics 3, 719 (1997).

5. T. Miyamoto, K. Takeuchi, F. Koyama, and K. Iga, IEEE Photonics Tech. Lett. 9, 1448 (1997).

6. Sarah R. Kurtz, D. Myers, and J. M. Olsen, in Proc. 26th IEEE Photovoltaics Spec. Conf. (IEEE, New York, 1997) pp. 875-878.

7. Steven R. Kurtz, A. A. Allerman, E. D. Jones, J. M. Gee, J. J. Banas, and B. E. Hammons, Appl. Phys. Lett. 74, 729 (1999).

8. E. V. K. Rao, A. Ougazzaden, Y. Le Bellego, and M. Juhel, Appl. Phys. Lett. 72, 1409 (1998).

9. M. Weyers and M. Sato, Appl. Phys. Lett. 62, p. 1396 (1993).

10. S. Miyoshi et al, J. Crystal Growth 124, 439 (1992).

11. A. Ougazzaden, Appl. Phys. Lett. 70, 2861 (1997).

12. D. J. Friedman, J. F. Geisz, S. R. Kurtz, J. M. Olson, R. Reedy, J. Crystal Growth, 195, 438 (1998)

13. J.F. Geisz, J. Crystal Growth 195, 401 (1998).

14. S. Kurtz, Appl. Phys. Lett., 74, 729 (1999).

15. D. Kwon, R. J. Kaplar, S. A. Ringel. A. A. Allerman, S. R. Kurtz, and E. D. Jones, Appl. Phys. Lett.74, 2830 (1999).

16. C. Tu (Private Communication).

17. J. F. Klem (Unpublished Results).

18. M. Cardona, in Modulation Spectroscopy, (Academic Press, New York, 1969)

19. W. Shan, W. Walukiewicz, J. W. Ager III, E. E. Haller, J. F. Geisz, D. J. Friedman, J. M. Olson and Sarah R. Kurtz, Phys. Rev. Lett. 82, 1221 (1999).

20. E. D. Jones, N. A. Modine, A. A. Allerman, I. J. Fritz, S. R. Kurtz, A. F. Wright, S. T. Tozer, and X. Wei, in Light-Emitting Diodes: Research, Manufacturing, and Applications III, edited by E. F. Schubert, I. T. Ferguson, and H. W. Yao, SPIE Conference Proceedings Vol. 3621 (International Society for Optical Engineering, Bellingham, WA, 1999), pp. 52-63.

21. W. Walukiewicz, J. Lagowski, L. Jastrzebski, M. Lichtensteiger and H. C. Gatos, J. Appl. Phys. 50, 899 (1979)

22. A. Rubio and M. L. Cohen, Phys. Rev. B 51, 4343(1995).

23. J. Neugebauer and C. G. Van de Walle, Phys. Rev. B 51, 10568 (1995).

24. S.-H. Wei and A. Zunger, Phys. Rev. Lett. 76, 664 (1996).

25. L. Bellaiche, S.-H. Wei, and A. Zunger, Phys. Rev. B 54, 17568 (1996). 
26. L. Bellaiche, S.-H. Wei, and A. Zunger, Appl. Phys. Lett. 70, 3558 (1997).

27. L. Bellaiche, S.-H. Wei, and A. Zunger, Phys. Rev. B 56, 10233 (1997).

28. L. Bellaiche and A. Zunger, Phys. Rev. B 57, 4425 (1998).

29. E. D. Jones, N. A. Modine, A. A. Allerman, S. R. Kurtz, A.F. Wright, S. T. Tozer, and X. Wei, to be published, Phys. Rev. B (July 15 1999).

30. V. Fiorentini, M. Methfessel, and M. Scheffier, Phys. Rev. B 47, 13353 (1993).

31. T. Mattila, S-H. Wei, and A. Zunger, to be published Phys Rev B.

32. D. J. Wolford, J. A. Bradley, K. Fry, and J. Thompson, in Physics of Semiconductors, ed. J. D. Chadi and W. A. Harrison (Springer, New York, 1984) pp. 627-630.

33. X. Liu, M. E. Pistol, L. Samuelson, S. Schwetlick, and W. Seifert, Appl. Phys. Lett. 56, 1451 (1990). 


\title{
NOVEL MATERIALS FOR PHOTOVOLTAIC TECHNOLOGIES
}

\author{
By \\ Paul Alivisatos, Berkeley \\ Sue Carter, UCSB \\ David Ginley, NREL \\ Gerald Meyer, Johns Hopkins \\ Arthur Nozik, NREL \\ Sandra Rosenthal, Vanderbilt
}

While existing photovoltaic technologies continue to advance, there are still many exciting opportunities in the area of novel materials. These opportunities arise because there is a substantial need for reducing the costs associated with the preparation and processing of photovoltaics, and because the theoretically possible photovoltaic efficiencies have yet to be achieved in practical devices. Thus it remains reasonable to continue photovoltaic research activity aimed at entirely new approaches to processing and at entirely new materials as the active media. This group identified three areas for further consideration:

1. Nano/molecular composites and hierarchical structures

2. Organic semiconductors

3. Hot carrier devices 


\section{Nano/molecular composites and hierarchical structures}

One of the greatest areas of opportunity in novel photovoltaics arises from developments in the physics and chemistry of nanometer scale components. Over the last decade there have been dramatic improvements in our understanding of the physical properties of nanocrystals, nanotubes, and nanorods; in parallel, there has been a great deal of progress in methods for the preparation and assembly of these objects into functional assemblies. Photovoltaics based upon the assembly of nanometer scale components may be advantageous for the following reasons:

1. Charge generation and separation take place on the nanometer scale, and thus assembly of components on the same length scale may permit detailed tailoring of properties;

2. Size dependent scaling laws allow for control of fundamental properties, for instance the optical density is enhanced in small semiconductor particles, as compared to the bulk, due to quantum confinement effects;

3. The use of nanoscale components can lead to many of the advantages observed in MBE grown systems (tandem cells), but with very inexpensive processing. Isolated nanoscale building blocks can be produced cheaply, with almost no defects;

Nanoscale components can be compatible with the elimination of toxic materials, and processing of large areas at low temperature.

There are several indications that these ideas are reasonable. Foremost among these is the recent development of the Graetzel cell, which is inexpensively assembled from nanoscale components, and which displays an efficiency of 10-12\%. In this cell, the individual components are assembled in a crude manner, and there is a great deal that could be gained by further systematic design. For instance, well-defined nanorods of two different semiconductors could be assembled in bundles, so that charge separation can take place at the interface between the rods, and then the individual charges can be conducted along the rods to the appropriate electrodes. By stacking layers of such devices, each layer with a different diameter of rod, a multi-band gap cell could be constructed, due to the quantum size effect. 
While the realization of such cells may still be a decade or more in the future, even in the short term there are potentially significant advantages to processing with nanoscale components now. Nanometer size crystals melt at lower temperatures than bulk crystals. Thus it is possible to spray nanocrystals onto a hot substrate, where they will fuse and form a tin film, at temperatures far lower than where thin films are grown by alternate methods. Further, such spray pyrolysis does not require vacuum, and thus is compatible with large area processing.

There are numerous areas of research that need to develop further before photovoltaic cells based on the assembly of nanoscale components can be realized. These include the ability to assemble components on multiple, hierarchical length scales, and the study of the physics of charge separation, electrical contact, band offsets, and electrical transport in composites of nanoscale components. This requires parallel developments in theory and experiment, in physics, chemistry, and materials science.

\section{Organic Photovoltaics}

Photovoltaics based on organic crystals, polymers, and molecules represent a potentially attractive approach to solar energy conversion. Organic materials offer the possibility of providing low cost, flexible, light weight, easily processed, environmentally friendly photovoltaics. The low power output realized from existing organic photovoltaic technologies has rendered them commercially non-viable. However, the wide range of materials that can be prepared and processed provides immense variability in composition that may one day be exploited to rationally design efficient organic photovoltaics.

The recent advent of commercially available light-emitting-diodes based on organic polymers and the development of processable semiconducting polymers with improved quality provide proof that organic materials can be used in photonic devices. If these or related materials are made to be viable in the photovoltaic industry, improved solar conversion efficiencies are urgently needed. Organic solar cells reported to date have less than $1 \%$ efficiencies under solar irradiation which compares poorly with 10-15 $\%$ of silicon photovoltaics. It is likely that new organic materials with small band gaps 
and high electron/hole mobilities are required for a future generation of efficient organic solar cells.

Organic materials may also provide applications in composite solar cells. One such application is in the sensitized nanocrystalline solar cells described in this chapter. Organic dyes with high oscillator strengths may be used in place of the widely used $\mathrm{Ru}(\mathrm{II})$ polypyridyl compounds. Furthermore, organic polymers or gels ("hole conductors') may be used in place of the iodide/triiodide electrolyte currently employed in the nanocrystalline solar cells. Other niche markets of organic materials already exist, and progress in these areas could help to lower costs and alleviate environmental problems for organic derived photovoltaic technologies.

In summary, organic solar cells offer many potential advantages over existing technologies but suffer from low power conversion efficiencies. The development of an efficient organic photovoltaic would provide the proof-of-concept required for industrial support and eventual commercialization. Organic materials may also find applications as components in more traditional photovoltaic devices.

\section{Hot Carrier PV Cells}

The maximum thermodynamic efficiency for converting solar radiation into electrical or chemical free energy is $32 \%$ for a single bandgap absorber if the photogenerated charge carriers (electrons and holes) are allowed to come to complete equilibrium with the lattice vibrations of the system (phonons in semiconductor materials). Carriers produced by absorption of photons with energies above the bandgap have excess kinetic energy and are called hot carriers; they have an electron or hole temperature greater than the lattice temperature. For solar radiation absorbed by semiconductors the initial photogenerated electron temperature is $3000 \mathrm{~K}$.

The equilibration (cooling) of the photogenerated carriers in semiconductors occurs via electron-phonon scattering and results in carrier cooling; in bulk semiconductor materials the process is very fast (a few picoseconds at solar intensities). Hence, the assumption of complete carrier-phonon equilibration in PV cells based on bulk semiconductor $p-n$ junctions is usually valid. This means that the excess kinetic 
energy of carriers created by absorption of photons having energies in excess of the bandgap is dissipated as heat by carrier-phonon interactions.

The optimum bandgap for single bandgap PV cells is $1.21 .4 \mathrm{eV}$; since solar photons have an energy range of 3.5 to $0.6 \mathrm{eV}$, a large fraction of the energy of absorbed supra-bandgap photons is lost as heat, resulting in the $32 \%$ limit on conversion efficiency. If this energy loss to lattice vibrations can be avoided; the thermodynamic efficiency of converting solar radiation to free energy is increased and reaches $66 \%$ in the limiting case of no carrier cooling loss. To reduce the thermalization loss in PV cells, the conventional approach has been to utilize tandem multi-bandgap structures to more closely match the absorption characteristic of the PV cell to the solar spectrum. The larger bandgap region absorbs the bigger photons and the smaller bandgap region absorbs the smaller photons. For 2- and 3-bandgap tandem structures the theoretical thermodynamic efficiencies are increased to 42 and $50 \%$, respectively.

Another way to avoid carrier cooling losses is to utilize the excess kinetic energy directly. This can be done in two ways: (1) extract the photogenerated carriers before they cool to produce a higher photovoltage; and (2) utilize the excess kinetic energy of hot carriers to produce a second electron-hole pair via an impact ionization process (reverse-Auger recombination).

The first approach requires that the rate of carrier transport and collection in the PV device be faster than carrier cooling; if the carrier cooling can be slowed this, of course, makes this process more competitive. Large reductions in carrier cooling rates have been observed in quantized semiconductor structures at high photoexcitation intensities. The second approach requires that the rate of impact ionization be greater that the rate of hot carrier cooling.

Since successful hot carrier processes in PV cells can produce much higher theoretical conversion efficiencies in single bandgap materials, their structure could be more simple than tandem solar cells and a research effort on these types of new PV cells 
is warranted. The problems that need to be addressed are: (1) how to slow hot carrier cooling in semiconductors at solar light intensities; (2) how to collect hot carriers efficiently at the contacts in hot carrier PV cells; and (3) how to enhance reverse-Auger processes to make them competitive with carrier cooling. 


\title{
TRANSPARENT CONDUCTING OXIDES: STATUS AND OPPORTUNITIES IN BASIC RESEARCH
}

\author{
Timothy J. Coutts ${ }^{1}$, Thomas O. Mason ${ }^{2}$, John D. Perkins ${ }^{1}$, and David S. Ginley ${ }^{1}$ \\ ${ }^{1}$ National Renewable Energy Laboratory, \\ 1617 Cole Blvd., Golden, CO 80401 \\ ${ }^{2}$ Northwestern University \\ Evanston, IL 60208
}

\begin{abstract}
In this paper, we begin by discussing the historical background of transparent conducting oxides and then make some general remarks about their typical properties. This is followed by a short discussion of the desired properties for future applications (particularly photovoltaic devices). These are ambitious objectives but they provide targets for future basic research and development. Although it may be possible to obtain these properties in the laboratory, it is vital to ensure that account is taken of industrial perceptions to the development of the next generation of materials. Hence, we spend some time discussing industrial criteria. Next, we discuss key physical properties that determine the macroscopic physical properties that, in turn, affect the performance of devices. Finally, we select several key topics that ought to be included in future basic research programs.
\end{abstract}

\section{INTRODUCTION}

Transparent conducting oxides (TCOs) have been used in several applications for three or four decades but, despite the huge volume of experience in the field, there remain many unanswered questions at both applied and fundamental levels. The reason that these issues have not been addressed, or only superficially so, is largely because the performance of the TCOs has been adequate to meet the demands of most applications considered thus far. For the purposes of the present document, the applications of concern are photovoltaic cells and modules, but the broader range of applications includes transparent electrode materials for both electrochromic cells and for liquid crystal display devices. In the case of both solar cells and the other two applications, improvement in performance of the TCOs is important because their non-ideal properties will eventually impact the performance of the complete device. We shall provide a definition of the term performance later in this document. From this point, we shall address the specific demands on TCOs imposed by prospective photovoltaic applications.

In the field of polycrystalline thin film solar cells, the dominant materials at present are copper indium gallium diselenide [1] (CIGS) and cadmium telluride [2] (CdTe). In addition, a-Si:H cells depend sensitively on the properties of the TCO transparent electrode. The first 
of the above cells is generally known as a substrate device and second as a superstrate device. The practical implication of this distinction is that the TCO element is either deposited directly onto the glass substrate before the absorber layers are deposited, or it is deposited on top of the semiconductor stack after all of the layers have been deposited. Although it is obvious that specific optical properties are required, additional important criteria must be met by the TCO and by the method used for its deposition. We shall discuss these later.

\section{$\underline{\text { General Observations }}$}

During the last thirty to forty years, the dominant TCOs have been tin oxide $\left(\mathrm{SnO}_{2}\right)$, indium oxide $\left(\mathrm{In}_{2} \mathrm{O}_{3}\right)$, indium tin oxide (ITO), and zinc oxide $(\mathrm{ZnO})$. All of these materials have been mass-produced in very large volumes over a long period of time and we assert that no new TCOs have been developed until about the last 5 years. During this time, there has been substantial coordinated activity in Japan, with Minami being particularly active [3]. Although efforts have been made elsewhere to develop new TCOs with the potential for improved performance [4-8], with the exception of a modest program at NREL from about 1985 onwards, a brief program at AT\&T Lucent Technologies in the mid-1990s, and a recent start-up program at Northwestern University, there have been very few concerted efforts in the United States.

The options are simple; to achieve superior performance, the TCO community must either discover new and improved materials or must find better ways of making the conventional TCOs mentioned above. We believe that the first of these is likely to prove far more fruitful than the second simply because of the huge number of publications devoted to optimization of TCO film deposition. It must also be recognized that there is a significant difference in the performance of the best material produced in research laboratories and those produced by manufacturing companies. While it is desirable to improve the performance of both, we must remember that the mass of coated glass (the usual substrate) produced by an average sized float glass melter is on the order of 600 tons/day [9]. Consequently, the deposition rate in large volume manufacture must necessarily be very rapid, compared with that typically used in laboratory R\&D. Although we believe that there are many basic research issues that ought to be addressed, we also recognize that the needs of the eventual large-scale manufacturer must be carefully considered.

A TCO is a wide band-gap semiconductor that has a relatively high concentration of free electrons in its conduction band. These arise either from defects in the material or from extrinsic dopants, the impurity levels of which lie near the conduction band edge. The highelectron-carrier concentration (the materials will be assumed to be n-type unless otherwise specified) causes absorption of electromagnetic radiation in both the visible and infrared portions of the spectrum For the present purposes, it is the former that is the more important. Because a TCO must necessarily represent a compromise between electrical conductivity and optical transmittance, a careful balance between the properties is required. Reduction of the resistivity involves either an increase in the carrier concentration or in the mobility. Increasing the former also leads to an increase in the visible absorption. Increasing the mobility, however, has no deleterious effect and is probably the best direction to follow. To 
achieve a high-carrier mobility will necessarily improve the optical properties. As we shall re-emphasize later, we see this as a key direction for future research and development of TCOs.

In present day, thin-film solar cells, both high- and low-resistivity materials are required to achieve maximum efficiencies. The role of the high-resistivity layer may be less obvious, but it appears that it is needed to prevent shunts of the junction leading to loss in voltage and fill factor. Optimization of the properties of TCOs generally requires an elevated temperature at some point in their fabrication. For example, some materials are deposited onto very hot substrates, which is compatible with glass manufacture, but some must be deposited onto heat-sensitive substrates such as plastics. For the latter, the upper limit on deposition or annealing temperature is probably less than $200^{\circ} \mathrm{C}$. In addition, in the CIGS substrate cell, zinc oxide is the last layer deposited, and its deposition temperature must be compatible with the semiconductor layers already deposited. If the TCO deposition temperature increases much above $250^{\circ} \mathrm{C}$, then interdiffusion of layers can occur, thereby ruining the device performance.

\section{$\underline{\text { Required Properties }}$}

At the start of a potentially new part of $R \& D$ into photovoltaics, it is necessary to define goals. The properties of TCOs that come most readily to mind are the electrical sheet resistance and the optical transmittance. These will be considered initially. However, other properties are likely to become very important to manufacturers of cells and modules and we shall discus those later in this section.

To a first approximation, we may suggest that a proper emphasis on novel TCOs should lead to a resistivity of less than $10^{-4} \Omega \mathrm{cm}$ (corresponding to a sheet resistance of less than $2 \Omega / \mathrm{G}$ for a film $0.5 \mu \mathrm{m}$ in thickness), together with maximal transmittance in the visible part of the spectrum. Although a sheet resistance of less than $10 / \mathrm{G}$ and a transmittance of greater than $80 \%$ in the visible part of the spectrum, may be acceptable for present-day solar cells, a more ambitious, more carefully defined target must be provided for future programmatic efforts. The definition of the desired electrical properties is straightforward but the definition of the optical properties needs more care. The reflectance and/or transmittance of thin TCO films exhibit interference fringes in the visible and, although the transmittance may peak at a value of $85-90 \%$, this is probably not a realistic average across the operational spectrum of typical devices (approximately 0.4-1 $\mu \mathrm{m}$ ). If instead, we define an absorption coefficient, this would be physically more useful. So, for example, if we have a film $0.5 \mu \mathrm{m}$ in thickness and its absorption coefficient is $2 \times 10^{3} \mathrm{~cm}^{-1}$ or less, then absorption will be a minimal problem. However, this performance should, ideally, be maintained across the entire operational wavelength range. Thus, a target of resistivity of $5 \times 10^{-5} \Omega \mathrm{cm}$ and a visible-absorption coefficient, due to free carriers, of $2 \times 10^{3} \mathrm{~cm}^{-1}$ or less are well beyond those achieved by any research laboratory and would be a difficult but feasible goal for a sustained, well-funded research program. In addition, these criteria must be met for a material with a band gap of at least $3 \mathrm{eV}$. 
Ideally, we would need a mobility of at least $100 \mathrm{~cm}^{2} \mathrm{~V}^{-1} \mathrm{~s}^{-1}$ to achieve these properties, and, in our opinion, it is on this quantity that the future research must focus. Typical mobilities of conventional semiconductors are generally less than $40 \mathrm{~cm}^{2} \mathrm{~V}^{-1} \mathrm{~s}^{-1}$, although there have been reports of higher values being achieved for cadmium stannate $\left(\mathrm{Cd}_{2} \mathrm{SnO}_{4}\right)$ [10].

In the next section, we shall comment on some industrial requirements that must also be met. If the novel materials developed by the $R \& D$ community are to be acceptable to industrial manufacturers of cells and modules, these need to be carefully considered. We shall continue by presenting a series of rhetorical questions concerning the key issues of charge scattering, the role of impurities and defects, and materials. We shall also include some input from theoreticians, which may be useful in seeking for new TCOs with potential applicability.

\section{INDUSTRIAL CONSIDERATIONS}

In its roadmap for the future, the glass industry has made it clear that they expect the manufacture of photovoltaic modules eventually to become a major product of the glass industry [9]. However, this is not presently the case. Assuming, however, that the roadmap prediction is realized, then one can appreciate that an extremely rapid rate of deposition must be used in order to handle the very large areas leaving the glass melter per unit time. Both sputtering and chemical vapor deposition, (CVD) can accommodate very large areas, and both can be designed for rapid rates of deposition. Of the two methods, CVD, probably has the lower capital cost and it is capable of producing mechanically tough films of large areas and acceptable (for present applications) electrical and optical properties. Film deposition may only take about thirty seconds to be compatible with the rate of deposition projected to be used in the manufacture of CdTe solar cells [11].

If sputtering emerges as the preferred deposition technique, then either metallic or metal oxide targets may be used. Metal targets are almost certainly cheaper to manufacture, but have a significant problem associated with them. In particular, during reactive sputtering, nodules tend to form on the target surface. These distort the distribution of the electric field across the surface and make the rate of erosion (and, consequently, the deposition rate as well) somewhat erratic. Progress has been made towards finding a solution to this problem by target manufacturers, who have used very dense sputtering targets. Nevertheless, the problem remains to some extent. If oxide targets are used, then these have their own problems. First of all, radio-frequency sputtering must be used. This is more expensive, more hazardous, and has a slower rate of deposition than CVD. The targets themselves are typically manufactured by pre-reacting stoichiometric mixtures of the individual oxides and then pressing these into targets. Occasionally, target suppliers manufacture hot-pressed targets that consist simply of the mixed oxides. The oxide targets are more expensive and present the issue of recycling. Although it is straightforward to reprocess a metal target, the same cannot be said of an oxide target.

Target manufacturers, particularly those of metal targets, are very sensitive to issues concerning toxicity. Even though the amount of toxic material (cadmium, for example) in a 
TCO film is very small, the perspective of the target manufacturers concerns the machining of the targets themselves and the need to protect machine operators (and ultimately the company itself) from the toxic metals therein. Strict safe operating procedures must be imposed and these represent cost, which clearly must be recovered through sales, thereby leading to higher target costs. Consequently, the issue of target toxicity is of great concern to the target manufacturers rather than to the end users of the TCO films.

The end-user of the TCO film, i.e., the cell/module manufacturer, will certainly demand that the film is readily amenable to etching or patterning, and must be simple to scribe with a laser. Chemical etching is problematic for tin oxide and this must be taken into account in the future development of novel TCOs.

The surfaces and interfaces of TCOs sometimes need to be smooth, as is the case with the CdTe and the CIGS solar cells, or hazy, i.e., textured, as is the case for -Si:H cells. The indirect band gap of the amorphous materials requires the films be textured to increase the path length through the absorber layer, thereby enhancing optical absorption. With CdTe and CIGS, the materials have direct band gaps and texturing is unnecessary. The interfaces of the TCO and other layers of the devices, e.g., CIGS, CdTe or CdS, must be stable and must also have a low-specific contact resistance. Film deposition sometimes requires an elevated substrate temperature. For a substrate cell, this will not affect the semiconductor layers to be deposited subsequently. This may not be the case for superstrate cells for which deposition and elevated temperatures may be necessary after all other semiconductor layers have been deposited. The performance of cells is usually dominated by interfacial regions and these must be carefully controlled. The contact resistance issue is not so severe because it is relatively easy to achieve values of less than some specified maximum permissible value (say, $10^{-2} \mathrm{~cm}^{2}$ ) or, when this is not the possible, other aspects actually dominate the parasitic losses in the device, as seems to be the case with the CdTe cell.

Finally, it is very likely that in mass production, manufacturers will accept critical production windows. Ideally, the deposition conditions must be reasonably forgiving and not require sensitive control.

\section{BASIC RESEARCH QUESTIONS AND OPPORTUNITIES}

In this section, we shall discuss some fundamental questions that must be answered if the projected performance figures defined earlier are ever to be realized. As was pointed out, the development of future TCOs must pay very careful attention to maximizing the carrier mobility. The first sub-section will raise a number of questions concerning the scattering processes. In many cases, the origin of the free carriers in TCOs is not clear. In some TCOs, defects such as oxygen vacancies may be responsible for the carriers. However, the donorlike oxygen vacancies must lie at rather shallow levels to give such high-carrier concentrations. Hence, the second sub-section deals with questions concerning defects and impurities. In the next sub-section, we raise some questions concerned specifically with materials. 
As mentioned above, the search for, fabrication of, and analyses of new TCO films must focus on achieving materials with higher electron mobilities. This can only be achieved by making material with longer electron relaxation times (i.e., by permitting the electrons to travel further between the successive randomizing collisions) or by identifying materials with lower electron-effective masses. It was pointed out long ago that attempts to increase the carrier relaxation time may prove fruitless because so many researchers, using many different deposition techniques, in the last thirty years or so have adjusted their deposition parameters to give optimal material. However, there has never been a clear example of a new deposition method, or set of deposition parameters, that led to significantly higher values of mobility than are usually reported. It was for this reason that our own investigations of cadmium stannate were first commenced. [5]. This material was pioneered by Nozik [10] and coworkers [12] in the early 1970s and it was suggested that the significantly higher mobilities achieved may have been associated with lower electron-effective masses. Although we have now achieved comparable mobilities, our detailed analyses of the transport properties indicate that the effective masses are similar to those reported for other TCOs. Coincidentally, however, the material does appear to lend itself to improved deposition techniques and the achievement of significantly higher values of relaxation time [13]. To this extent, we obtained the right result for the wrong reason! Despite this, we still suggest that an appropriate role for solid-state theorists would be to construct band diagrams and estimate effective masses of likely new TCOs identified on the basis of other theoretical criteria. Ionized impurities have often been identified as the likely scattering centers, but in very few cases have steps been taken to prove this assertion. In our own work, we have used the socalled method of four coefficients [14] in which the conductivity, Hall coefficient, Seebeck coefficient, and Nernst coefficient, are measured on the same sample without any change in the configuration. This enables not only the effective mass and the relaxation time to be calculated directly, but also gives the scattering parameter that relates electron relaxation time to Fermi energy. This work has also established that the conduction band is parabolic to within experimental uncertainly. Additional theoretical work is needed to show that this is expected from ab initio arguments.

In polycrystalline materials, it is often presumed that grain boundaries scatter charge carriers and cause a reduction in mobility relative to single crystals. Potentially, the grain boundaries may be effective scatterers of charge but, in reality, we do not believe that this is necessarily the case. For a heavily degenerate semiconductor, the electron mean-free path is on the order of $4 \mathrm{~nm}$. This is much less than typical grain sizes leading us to the tentative conclusion that grain boundary scattering is probably unimportant. We have also never observed a significant variation with frequency of the ac impedance of the film, lending further support to the notion that the grain boundaries are shunted. Finally, the degeneracy of the material (i.e., the energy separation between the Fermi level and the conduction-band minimum) can be as high as $0.7 \mathrm{eV}$, which is probably larger than the height of the grain boundary potential barrier. For these three reasons, we have reached the conclusion that grain boundaries are probably unimportant in TCO films. However, this requires separate confirmation, and we encourage other researchers in the field to test this hypothesis. It would be interesting, for example, to work at lower carrier concentrations for which grain boundary scattering may be expected to play a much stronger role, and to compare its behavior with theories of grain 
boundary scattering [15] and scattering by ionized impurities [16], neutral impurities [17] and other mechanisms.

At high electron concentrations, $\left(10^{20}-10^{21} \mathrm{~cm}^{-3}\right)$ it is highly probable that compensation will occur. So far as we are aware, this has not been taken into account in any assessment of TCOs. This is, therefore, an important direction for future theoretical and experimental investigation.

Finally, at high-electron concentrations, screening of impurity ions will likely occur. The positively charged ions gather a cloud of electrons around them, thus restricting their electric field to the immediate vicinity of the cloud. Calculations of the role of screening have been made that suggest higher mobilities may be achievable than have been predicted to date on the basis of ionized impurity scattering alone. Hence, future theoretical investigations of charge transport should include the role of both compensation and ionized impurity screening. If one discounts the role of grain boundaries as a scattering species, then we must address the role of ionized impurities or some other mechanism.

If scattering occurs within the grains rather than at the grain boundaries, then it is important to identify the mechanism of scattering. If dislocation networks form, then it is important to know how they act electrically and whether their contribution may be reduced by improved fabrication methods. As mentioned earlier, it has often been speculated that oxygen vacancies give rise to the free electrons in the conduction band of the TCOs. This raises the interesting question of whether high mobilities could be achieved in stoichiometric films extrinsically doped. Examples of extrinsically doped TCOs include zinc oxide and tin oxide doped cationically with aluminum and antimony respectively, and anionically with fluorine. Nevertheless, exceptionally high mobilities have not been observed, although the films may not have been precisely stoichiometric. Our own work on cadmium stannate has led to high mobilities (relative to other TCOs) and these films have been made without extrinsic doping. Although our results are promising, in reality we know neither the source of the carriers nor the dominant scattering mechanism [13]. It would be interesting to investigate cadmium stannate and, perhaps, other materials in the spinel family using extrinsic doping to establish the mobility at even higher carrier concentrations. This, of course, presumes that compensation does not overwhelm the additional donors.

Single crystals of several TCOs have been made [18-21] and very much higher mobilities have been demonstrated than have ever been achieved in thin polycrystalline films. Although the temptation is to conclude that the difference is due to grain boundary scattering, for the reasons outlined above, we are inclined to discount this possibility. It appears to be more likely that the increased mobility is due to improved material quality. That being the case, it may still be possible to improve the quality of polycrystalline TCOs, thereby increasing their mobility. However, single crystal TCOs need to be manufactured in order to support these studies. The crystals should be large enough to permit characterization using the standard techniques but, in addition, we believe that a wider range of characterization techniques may be required. The crystal sizes, therefore, need to be amenable to these. The four coefficient method used at NREL, for example, requires minimum areas of approximately $7 \times 3 \mathrm{~mm}$. Most work on single crystal growth has led to samples that are dendritic and very difficult to characterize electrically and optically. 


\section{The Nature of Defects and Impurities}

In summary, there are many interesting and potentially valuable avenues of research open to much more detailed research and development than has been conducted on TCOs in their history to date.

As discussed earlier, the origin of the electrons in unintentional doped TCO films is far from clear. Although many papers state that these are associated with oxygen vacancies, it is perhaps surprising that these lie at shallow levels, relative to the conduction-band minimum, rather than at deeper levels as is found for other native defects in other semiconductors. With most semiconductors, it is more efficient to dope stoichiometric material extrinsically rather than relying on the native defects. Although this has been done in $\mathrm{ZnO}: \mathrm{F}$ and $\operatorname{In}_{2} \mathrm{O}_{3}: \mathrm{Sn}$, extrinsic doping has not been used with most TCOs and particularly those developed recently. Therefore, we recommend that some attention be paid to the deliberate doping of TCO films. This could be performed during film deposition or subsequent to deposition with the former preferred.

\section{MATERIALS}

In the CdTe superstrate cell, the incident light passes first through the glass substrate, next to the TCO film, and then into the p-CdTe/CdS layers of the junction itself. Exactly the same happens with a-Si:H cells. In both cases, therefore, a reverse-bias junction between the ntype TCO and the p-type absorber layer must be formed, and the properties of this may affect the performance of the device to a greater or lesser extent. However, the extent is unknown, but incorporating a p-type TCO at the front surface could solve this problem. However, except for one or two notable exceptions, there have been no reports of successful fabrication of p-type materials. Given the inverse dependence of the mobility on effective mass, one may expect that the electrical and optical performance of p-type TCOs to be inferior to those of n-type films with their much lower electron effective masses. However, all this needs to be determined by both theory and experiment, and we suggest that this work is urgently needed.

The subject of co-doping has been discussed in the literature during the last one to two years, and it has had very interesting effects that may be useful in the development of p-type TCOs. There are some notable examples of materials being doped with a carrier type opposite to that which is normally observed. For example, high conductivity n-type diamond films have been made by Nishmori et al. [22] and p-type GaN was made by Yamamoto et al. using either Be or $\mathrm{Mg}$ acceptors with either $\mathrm{Si}$ or $\mathrm{O}$ as the donor co-dopants [23]. Yamamoto et al also proposed using the co-doping approach for the production of p-type $\mathrm{CuInS}_{2}$ [24]. In the present context, it is also interesting to note that Yamamoto et al proposed to use the codoping method for the fabrication of p-type $\mathrm{ZnO}$ [25] This work was, however, theoretical and so far as we are aware, has not yet been reduced to practice. We suggest that systematic studies of the use of co-doping in the fabrication of p-type TCOs be initiated. Given their potential advantages, this is a topic we chose to emphasize later in the next section, dealing with key recommendations. First, we shall deal with some general criteria that may be useful in searching for new and improved TCOs. 
As implicit in the name, viable transparent conductors must be simultaneously transparent and conducting, an unusual combination. To date, this combination has only been usefully realized in a limited class of metal oxides, all of which yield n-type conductors. By comparing a variety of known materials we can establish some general guidelines that may be useful in searching for new transparent conductors, possibly including p-type transparent conductors and non-oxide transparent conductors.

Stoichiometric $\operatorname{In}_{2} \mathrm{O}_{3}$ is a transparent insulator that can be doped by substituting $\mathrm{Sn}$ for In to yield n-type indium tin oxide (ITO), a well-known transparent conductor. In contrast, $\mathrm{Al}_{2} \mathrm{O}_{3}$, which is isoelectronic with indium oxide and is another transparent insulator, can not be effectively doped. A major difference between these two materials is that In is a multivalent cation as evidenced by the existence of several indium oxides: $\operatorname{In}_{2} \mathrm{O}_{3}, \operatorname{InO}$ and $\operatorname{In}_{2} \mathrm{O}$. In a simple integer-valence ionic model, the In valence increases from +3 to +2 to +1 in this sequence. Hence, the multivalent nature of In likely enables the $\operatorname{In}_{2} \mathrm{O}_{3}$ host electronic structure to accommodate the enormous conduction electron concentrations (of order $10^{20} / \mathrm{cm}^{3}$ or more) typical of useful transparent conductors, and associated with the three indium oxides mentioned above.

Considering only the multivalence cation criterion, one might expect $\mathrm{WO}_{3}$ to be an excellent TCO host. Stochiometric $\mathrm{WO}_{3}$ is transparent, with a nominal $\mathrm{W}$ valence of +6 . Compounds with tungsten ion valences of $+6,+5,+4,+3$ and +2 are generally considered stable. However, when $\mathrm{WO}_{3}$ is electron doped with either oxygen vacancies or $\mathrm{Li}$ intercalation to form $\mathrm{Li}_{\mathrm{x}} \mathrm{WO}_{3}$, the film quickly darkens. Hence, its use in electrochromic devices. $\mathrm{WO}_{3}$ can accommodate high-conduction electron concentrations but does not remain transparent. The multivalency is therefore not a sufficient condition for a material to form a TCO.

Again, considering a simple integer-valence ionic model, tungsten in $\mathrm{WO}_{3}$ has electronic configuration $\mathrm{W}^{6+}$ : $[\mathrm{Xe}] 4 \mathrm{f}^{14} 5 \mathrm{~d}^{0} 6 \mathrm{~s}^{0}$ whereas indium in $\mathrm{In}_{2} \mathrm{O}_{3}$ has electronic configuration $\mathrm{In}^{3+}$ : $[\mathrm{Kr}] 4 \mathrm{~d}^{10} 5 \mathrm{~s}^{0} 5 \mathrm{p}^{0}$. Hence, adding electrons to $\mathrm{W}^{6+}$ adds electrons to the energetically clustered $5 \mathrm{~d}$ levels, whereas adding electrons to $\mathrm{In}^{3+}$ adds electrons to the empty $5 \mathrm{~s}$ and $5 \mathrm{p}$ levels. Of course, substantial metal/oxygen hybridization occurs but, in general, adding electrons to energetically clustered bands will probably introduce optical transitions in the visible portion of the spectrum. Hence, for n-type transparent conductors, the conduction band minimum should be well separated energetically from higher lying bands. This condition is met in the elements $\mathrm{Cd}, \mathrm{In}, \mathrm{Sn}, \mathrm{Zn}$ and $\mathrm{Ga}$, all of which still have filled d-bands after reaction with oxygen.

It is also important that anion vacancies act as shallow donor levels, rather than as deep traps. In alkali-halides, anion vacancies yield electron traps that are deep enough for transitions between the conduction band and the traps to occur, with associated absorption bands, in the visible. Similar absorption bands exist in alkali-earth metal oxides such as $\mathrm{CaO}, \mathrm{SrO}$ and $\mathrm{BaO}$. Not only can such spatially localized electrons yield coloring optical transitions, but they will also not yield metallic conduction. 
The preceding discussion considered only n-type materials. However, p-type transparent conductors would be very useful for the reasons given earlier. Actually, conducting p-type metal oxides are very well known; almost all copper oxide-based, high-temperature superconductors are p-type conductors derived from semiconducting hosts such as $\mathrm{La}_{2} \mathrm{CuO}_{4}$, which has a band gap of about $2 \mathrm{eV}$. However, these materials and many other stoichiometric transition metal oxides, are correlated electron insulators, rather than filled-band insulators. Not only do these materials generally become broadly absorbing when doped, but even when perfectly stochiometric, sub-band-gap optical excitations exist due to coupled vibrational, magnetic, and excitonic transitions. Hence, correlated electron insulators are not potential host materials for transparent conductors.

Suggestions have recently been made that two other copper oxide-like materials may be ptype transparent conductors. These are $\mathrm{CuAlO}_{2}$ and $\mathrm{SrCu}_{2} \mathrm{O}_{2}$. Both of these are p-type transparent thin films that have been successfully made, but with carrier densities of order $10^{17} / \mathrm{cm}^{3}$ : much lower than n-type materials. It is interesting to consider these two materials in light of the preceding discussion. Again, considering a simple inter-valence ionic model, the $\mathrm{Cu}$ ion is $\mathrm{Cu}^{1+}:[\mathrm{Ar}] 3 \mathrm{~d}^{10} 4 \mathrm{~s}^{0}$, in both materials, making these materials filled $\mathrm{d}$-band insulators, rather than correlated electron insulators. Furthermore, two copper oxides are well known, $\mathrm{Cu}_{2} \mathrm{O}\left(\mathrm{Cu}^{1+}\right)$ and $\mathrm{CuO}\left(\mathrm{Cu}^{2+}\right)$. Hence, in $\mathrm{CuAlO}_{2}$, the multi-valent cation criterion may imply that the material can accommodate p-type doping. However, adding holes to $\mathrm{Cu}^{1+}$ adds holes to the $\mathrm{Cu}$ d-states, which is analogous to adding electrons to $\mathrm{WO}_{3}$ and leads to strong absorption. There are two possible explanations for the observed transparency of p-type $\mathrm{CuAlO}_{2}$ made to date, 1) the doping level is so low that, in thin-film form, the absorption is not strong enough to cause coloration or 2) that strong $\mathrm{Cu}-\mathrm{O}$ hybridization significantly alters the optical excitations due to hole doping in $\mathrm{CuAlO}_{2}$ compared to electron doping into $\mathrm{WO}_{3}$.

We believe that criteria such as the above may help establish potentially new TCO materials that may be made either in thin-film form or in bulk samples. They do not, of course, provide any guidance on whether or not the materials will have improved electro-optical properties. Next, we shall consider the use of bulk-phase diagrams, which also appears to have considerable promise in identifying novel TCOs.

We may expect future TCOs to be relatively complex structures with several cations and completely new phases. A good example of the search for novel materials is provided by the group from Northwestern University [26] which has performed a systematic investigation of oxide-phase space bounded by the known TCO species-In, Zn, Sn, Cd, and Ga. The search has followed the guidelines discovered above. The novel TCO phases discovered to date suggest possibilities for enhanced mobility materials. Firstly, 2-dimensional layered structures such as the homologous series of $\operatorname{In}_{2} \mathrm{O}_{3}(\mathrm{ZnO})_{\mathrm{k}}(\mathrm{k}=1,2,3,4,5,7,11)$ phases (27) suggest the possibility of preferential doping on "spacer" layers, with carrier injection into unperturbed conduction layers. Moriga [28], commented that the $\mathrm{k}=1$, and $\mathrm{k}=2$ members may only be stabilized by partial substitution of Ga for In. Secondly, co-substitution may be an effective means of altering TCO properties. This could lead to enhanced mobility along the conduction layers because they are otherwise undoped, and therefore, free of ionized impurity scattering The extensive 40 at. \% co-substitution of $\mathrm{Zn}^{2+} / \mathrm{Sn}^{4+}$ for two $\mathrm{In}^{3+}$ in $\mathrm{In}_{2} \mathrm{O}_{3}$ (versus only 6 at. \% Sn substitution in ITO) results in excellent optical properties with only minor reductions in conductivity [29]. The analogous $\mathrm{Cd}^{2+} / \mathrm{Sn}^{4+}$ co-substituted in $\mathrm{In}_{2} \mathrm{O}_{3}$ is under investigation by the Northwestern University group. Two other strategies that have 
emerged from this work concern electron hybridization, i.e., crystal structures with more than one cation site. For example, the first true-ternary compound TCO to be reported, $\mathrm{Ga}_{3} \mathrm{In}_{5} \mathrm{Sn}_{2} \mathrm{O}_{16}$ [30] has three of the key cations as structural components, rather than as dopants. These have 4-fold, 6-fold, and 7-fold coordination environments. The ordering between the various sites is believed to be important in governing the electrical and optical properties of the TCO. Unpublished work by the Northwestern University group shows the existence of a spinel solid solution, (1-x) $\mathrm{CdIn}_{2} \mathrm{O}_{4}-\mathrm{x} \mathrm{Cd}_{2} \mathrm{SnO}_{4}$, over the range 0 x 0.75 . Again, the distribution of cations between tetrahedral and octahedral sites is believed to play an important role in determining the TCO properties.

It ought to be stressed that most of these new TCO phases have yet to be deposited as highquality think films. The bulk resistivities are typically in the $10^{-3}-10^{-2} \mathrm{~cm}$ range; however, the above mentioned solid solution exhibits conductivities approaching $3 \times 10^{-4} \mathrm{~cm}$, which is comparable with, or superior to, that of ITO made by the same solid-state reaction method. In general, the properties of thin films are superior to those of bulk materials. There are two other key differences between bulk and films. Often, the solubility limits can be greatly enhanced in films, e.g., ITO, that incorporates only $\sim 6$ at. $\%$ in bulk, but several times this in films, depending on deposition conditions. Secondly, phases that are unstable in bulk can be metastabilized as films. The spinel phase of $\mathrm{Cd}_{2} \mathrm{SnO}_{4}$ is a good example of this behavior because it exists as a film but not as a bulk material. Note that $\mathrm{Cd}_{2} \mathrm{SnO}_{4}$ corresponds to $\mathrm{x}=1$ in the solid solution mentioned above, which is only stable for values of $x<0.75$. The potential for novel stable and metastable phases in the unexplored phase space of the In- $\mathrm{Zn}$ $\mathrm{Sn}-\mathrm{Cd}-\mathrm{Ga}-\mathrm{O}$ system is very considerable, in our opinion.

\section{KEY RECOMMENDATIONS FOR BASIC RESEARCH}

Rather than presenting a summary of the foregoing discussion, we shall simply itemize the key topics for basic research and development: these being issues that we believe must be addressed for the development of next-generation materials that will be crucial for more demanding applications. This is not intended to be an exhaustive list but rather one that makes specific reference to earlier discussions in this paper.

- Materials' science issues

-use $a b$ initio calculations and empirical models in the search new materials -use bulk phase diagrams to identify new phases in binary and ternary TCOs -explore the importance of metastability and crystallinity -explore more complex oxide mixtures -study compounds other than oxides for required optical and electrical properties -develop highly conducting (say, resistivity less than $10^{-2} \mathrm{~cm}$ ) p-type materials -use a wider range of characterization methods to analyze films and crystals -develop methods of deposition to give high rates and low substrate temperatures -investigate the use of single crystal compounds and alloys to assist evaluation of intrinsic properties

-investigate new approaches to the synthesis of device-ready materials 
- Investigate scattering mechanisms

-develop methodologies with which to identify materials with prospectively good electro-optical properties

-use transport measurements to determine predominant scattering mechanism and to probe the band structure of the semiconductors

- establish the upper limit in mobility

-investigate screening effects

-model and minimize the effects of neutral impurities and compensation

-investigate the role of dipole fields on scattering

-evaluate the importance of grain boundaries and linear defects

- Investigate the role of impurities and defects

-establish the role of oxygen vacancies

-investigate co-doping of films and crystals

-determine the importance of non-ionized impurities

-learn how to increase ionization efficiency of impurities

\section{ACKNOWLEDGEMENTS}

The authors would like to acknowledge the contributions made to this paper by Professor Roy Gordon of Harvard University, Dr. Brian Lewis of Arconium, Inc., Dr. Clark Bright of DeltaV Technologies, Inc., Professor Victor Kaydanov of Colorado School of Mines, Drs. Alex Zunger, and Kannan Ramanathan, Peter Sheldon, Bolko von Roedern and Ken Zweibel, all of NREL. This work was supported by the US DoE through Contract No. DE-AC36-98GO10337. 


\section{REFERENCES}

(1) Kessler, J. et al., (1993), 'Interface engineering between $\mathrm{CuInSe}_{2}$ and $\mathrm{ZnO}$ ', in the Twenty third IEEE Photovoltaic Specialists Conference, 447-452, IEEE, Piscataway, NJ.

(2) Zweibel, K., Ullal, H. S. and von Roedern, B., (1996), 'Progress and issues in polycrystalline thin-film PV technologies', in the Twenty Fifth IEEE Photovoltaic Specialists Conference, 745-750, IEEE, Piscataway, NJ.

(3) Minami, T., Kakumu, T. and Takata, S., 'Preparation of transparent and conductive $\mathrm{In}_{2} \mathrm{O}_{3} / \mathrm{ZnO}$ films by radio frequency magnetron sputtering', Journal of Vacuum Science \& Technology A (Vacuum, Surfaces, and Films) Conference Title: J. Vac. Sci. Technol. A, Vac. Surf. Films (USA) 14, 1704-1708, (1996).

(4) Dhere, R. G. et al., 'Electro-optical properties of thin indium tin oxide films: limitations on performance', Solar Cells 21, 281-290, (1987).

(5) Coutts, T. J., Wu, X. and Mulligan, W. P., 'High performance transparent conducting oxides based on cadmium stannate', Journal of Electronic Materials 25, 935-943, (1996).

(6) Phillips, J. M. et al., 'Transparent conducting thin-films of $\mathrm{GaInO}_{3}$ ', Applied Physics Letters 65, 115-117, (1994).

(7) Cava, R. J. et al., 'GaInO 3 - A New Transparent Conducting Oxide', Applied Physics Letters 64, 2071-2072, (1994).

(8) Palmer, G. B., Poeppelmeier, K. R. and Mason, T. O., ' $\mathrm{Zn}_{2-\mathrm{x}} \mathrm{Sn}_{1-\mathrm{x}} \mathrm{In}_{2 \mathrm{x}} \mathrm{O}_{4-\delta}$ :An indium-substituted spinel with transparent conducting properties', Journal of Solid State Chemistry 134, 192-197, (1997).

(9) Eisenhauer, J. et al. (1997), Glass technology roadmap, Department of Energy Workshop Report No. Conf-9704153, Energetics, Inc., Columbia, Maryland.

(10) Nozik, A. J., 'Optical and Electrical Properties of $\mathrm{Cd}_{2} \mathrm{SnO}_{4}$ : A Defect Semiconductor', Phys. Rev. B 6, 453-459, (1972).

(11) Powell, R. C., Dorer, G. L., Jayamaha, U. and Hanak, J. J. (1998), Technology support for initiation of high-throughput processing of thin-film PV modules, Final Technical Report No. NREL/SR-520-25422, National Renewable Energy Laboratory, Golden, CO 80401.

(12) Haacke, G., Mealmaker, W. E. and Siegel, L. A., 'Sputter Deposition and Characterization of $\mathrm{Cd}_{2} \mathrm{SnO}_{4}$ Films', Thin Solid Films 55, 67-81, (1978).

(13) Mulligan, W. P., (1997), 'A Study of the Fundamental Limits to Electron Mobility in Cadmium Stannate Thin Films', Ph.D. thesis, Colorado School of Mines.

(14) Chernik, I. A., Kaidanov, V. I. and Vinogradova, M. I., 'Investigation of the valence band of lead telluride using transport phenomena', Soviet Physics-Semiconductors 2, 645651, (1968).

(15) Seto, J., 'The electrical properties of polycrystalline silicon films', Journal of Applied Physics 46, 5247-5254, (1975).

(16) Conwell, E. and F., W. V., 'Theory of impurity scattering in semiconductors', Physical Review 77, 388-390, (1950).

(17) Erginsoy, C., 'Neutral impurity scattering in semiconductors', Physical Review 79, 1013-1014, (1950).

(18) Kanai, Y., 'Electrical properties of indium-tin-oxide single crystals', Japanese Journal of Applied Physics 23, L12-14, (1984).

(19) Kanai, Y., 'Electrical properties of $\mathrm{In}_{2} \mathrm{O}_{3}$ single crystals doped with metallic donor impurity', Japanese Journal of Applied Physics 23, 127-127, (1984). 
(20) Marley, J. A. and Dockerty, R. C., 'Electrical properties of stannic oxide single crystals', Physical Review 140, A 304- A 310, (1965).

(21) Wen, S. J., Couturier, G., Chaminade, J. P., Marquestaut, E. and Claverie, J. H., P., 'Electrical of pure $\mathrm{In}_{2} \mathrm{O}_{3}$ and $\mathrm{Sn}$-doped $\mathrm{In}_{2} \mathrm{O}_{3}$ single crystals and ceramics', Journal of Solid State Chemistry 101, 203-210, (1992).

(22) Nishimori, T., Nakano, K. and Sakamoto, H., 'n-type high-conductive epitaxial diamond film prepared by gas source molecular beam epitaxy with methane and tri-nbutylphosphine', Applied Physics Letters 71, 945-947, (1997).

(23) Yamamoto, T. and Katayama-Yoshida, H., 'Electronic structures of p-type GaN codoped with $\mathrm{Be}$ or $\mathrm{Mg}$ as the acceptors and $\mathrm{Si}$ or $\mathrm{O}$ as the donor codopants', Journal of Crystal Growth 189/190, 532-536, (1998).

(24) Yamamoto, T. and Katayama-Yoshida, H., (1998), 'A codoping method in $\mathrm{CuInS}_{2}$ proposed by ab initio electronic structure calculations', in the 11th. Int. Conference on Ternary and Multinary Compounds, ICTMC-11, 152, 37-40, IOP Publishing Ltd., Bristol, U. $\mathrm{K}$.

(25) Yamamoto, T. and Katayama-Yoshida, H., 'Solution using a codoping method to unipolarity for the fabrication of p-type ZnO', Japanese J. of Applied Physics 38, L166L169, (1999).

(26) Kammler, D. R. et al., (1999), 'Novel compound and solid solution transparent conducting oxides for photovoltaics', in the 195th. Meeting of the Electrochemical Society, to be published, The Electrochemical Society, Pennington, NJ.

(27) Moriga, T., Edwards, D. D., Mason, T. O., Palmer, G. B. and Poeppelmeier, K. R., 'Phase relationships and physical properties of homologous compounds in the $\mathrm{In}_{2} \mathrm{O}_{3}-\mathrm{ZnO}$ system', Journal of the American Ceramic Society 81, 1310-, (1998).

(28) Moriga, T., Kammler, D., Mason, T. O., Palmer, G. B. and Poeppelmeier, K. R., 'Electrical and optical properties of transparent-conducting homologous compounds in the indium-gallium-zinc oxide system', Journal of the American Ceramics Society in press,(1999).

(29) Palmer, G. B., Poeppelmeier, K. R. and Mason, T. O., 'Conductivity and transparency of $\mathrm{ZnO} / \mathrm{SnO} 2-$ cosubstituted In2O3', Chemistry of Materials 9, 3121-3126, (1997).

(30) Edwards, D. D., Mason, T. O., Goutenoire, F. and Poeppelmeier, K. R., 'A new transparent conducting oxide in the $\mathrm{Ga}_{2} \mathrm{O}_{3}-\mathrm{In}_{2} \mathrm{O}_{3}-\mathrm{SnO}_{2}$ system', Applied Physics Letters 70, 1706-1708, (1997). 


\title{
PHOTOVOLTAICS CHARACTERIZATION: AN OVERVIEW
}

\author{
Y.H. Kao \\ Department of Physics \\ State University of New York at Buffalo \\ Amherst, NY 14260 \\ Lawrence Kazmerski \\ National Renewable Energy Laboratory \\ Golden, Colorado 80401 \\ Kelvin G. Lynn \\ Materials Research Center \\ Washington State University \\ Pullman, WA 99164-2711 \\ Angelo Mascarenhas \\ National Renewable Energy Laboratory \\ Golden, Colorado 80401
}

\begin{abstract}
In order to move to a next generation of characterization methods, the requirements must be fully understood and documented. Presented herein are the required functionalities for the next generation of characterization methods. This paper is not meant to be exhaustive, but instead presents new developing characterization methods. The basic characterization requirements are outlined in the introduction. It is expected that in the future, phenomena will be understood on the atomic scale and applied to large-scale arrays for a complete understanding of the various affects that determine the real cell efficiency. There is a need for a fundamental understanding from atomic and nanoscale characterizations of impurities, native defects, extended defects and interfaces to provide the necessary understanding of these types of PV cells. This information from fundamental probes should be used for input to the performance characterization of the developing technologies, which include high-flux operation, multijunction and lower band-gap systems. These methods will allow new materials to come to realization at a much faster rate than was possible in previous years.
\end{abstract}




\section{INTRODUCTION}

Materials characterization plays a pivotal role in photovoltaic (PV) research and is essential in realizing the breadth of new technologies on the horizon. A number of techniques from atoms to arrays are currently available to determine the structure and properties of PV materials, devices, and systems. However, a clear understanding of the relationship between the underlying materials' characteristics and device performance is still lacking. Efforts and techniques should be devoted to the identification of a set of physical parameters, which can be quantitatively correlated with actual solar cell performance using nondestructive and in-situ characterization techniques. Special electrooptical characterization methods, such as minority-carrier parameter determinations and chemical defect resolutions are needed for the next generation of industrial tools. Figures of merit, namely, minority-carrier lifetime, interfacial recombination velocity, and especially room temperature efficiency, still need to be improved. A serious modeling effort is a necessary component to achieve the final solution. This will require a combined and collaborative effort on the part of industry, national labs, and universities working hand-in-hand to develop the necessary understanding from point defects and impurity complexes to arrays of solar energy panels.

To understand compensation by native defects and/or unintentional impurities, and, in the case of polycrystalline material, grain boundary defects and impurities, leads to understanding of real cell efficiencies. The final solution can be achieved only with a combination of new characterization methods to input the necessary details and industry to use the understanding to make cost-effective solutions for the next generation of PV devices for the United States and the world.

The determination of the device parameters of solar cells and modules is essential to the establishment and tracking of the progress in this arena. When performed under standard conditions, this also provides the mechanism to objectively compare various PV technologies. Finally, since the "device" is the proof of any PV material system, the determination and demonstration of device performance is important for establishing credibility, and providing guidance for further development. The accurate, rapid, and precise determination of efficiency and related device parameters has become an essential part of solar cell research, development, and manufacturing.

A significant need for a centralized, independent laboratory facility has proved to be a requirement for the credible evaluation of photovoltaic devices. Constant inputs are needed for new and unique front-end research methods to provide industry with the necessary input for evaluation, but an understanding of the process is also important. The centralization of the standard measurement capability has also helped to disseminate standard test methods and reporting conditions within the photovoltaic community. It has led to international intercomparisons to make sure that efficiencies measured in one part of the world track those in other locations. Almost all of these central and standard performance-evaluation laboratories can trace their standards and references to common sources. However, new measurement techniques need to be generated in order to understand and improve cell efficiencies. 
In the near term (next 5-10 years), a number of areas of performance evaluation are already in development or identified, as needed to meet anticipated needs. The ability to determine and compare the operational characteristics of solar cells and modules has progressed significantly over the past 15 years, especially with the adoption of international standards for measuring and reporting efficiencies and the initiation of certification procedures and laboratories under the PV (Photovoltaic) GaP program. With the guidance of the PV Program's Five-Year Plan and the recent PV Industry Roadmap, coupled with the experience of those working in this field, several needs are identifiable:

Precision and Accuracy. Measurement uncertainty analysis has been applied to every step of the standard cell and module evaluation processes. Work continues to refine the techniques to provide customers with the best possible evaluation of parameters. The best current methods at the standards laboratories have accuracies (or uncertainties in the efficiency with respect to standard conditions) of $\pm 1 \%$ for single-junction cells, $\pm 5 \%$ for concentrator cells, and $\pm 3 \%$ for modules. For multijunction devices, these range from $\pm 3 \%$ for 1 -sun cells to about $\pm 5 \%$ for modules. The single-junction, 1-sun cell uncertainties are acceptable. The need for concentrator and module technology development requires refinement in those techniques in order to provide better comparisons and evaluation of progress with these technologies.

Standards. New and novel device technologies require rating methodologies. Examples include bifacial cells and holographic concentrators. Many alternatives to the standard "accepted" reporting techniques must be evaluated for their utility and accuracy. In general, standards activities require almost constant attention to ensure equity for evolving technologies. For example, qualification tests developed and accepted for crystalline Si modules might not be appropriate for thin-film technologies.

Standard test and reporting conditions must be checked periodically, as the range of devices in the photovoltaic portfolio expands. On the module side, the development and implementation of other rating schemes (power, energy, etc.). Alternatives to efficiency are required as more appropriate for this component area.

Concentrators. With some renewed interest in these technologies (with some added focus in the High-Performance Photovoltaics Initiative for the coming 10-year period), the current state-of-the-art is not prepared to meet the needs for many of these device evaluations. Issues relating to linearity must be resolved to adequately and fairly evaluate these technologies. Methods for determining efficiencies in the 30\%-35\% range are currently lacking from precision, time-to-perform (responsiveness), reproducibility, and controllability aspects. Much time has been devoted to the development and refinement of the measurement of these concentrator cells as with AM1.5 technologies. There exists a whole range of concentrators in the 20-50 concentrator range that use a substantial portion of diffuse radiation. This has not been standardized, and procedures to compare the performance at such levels (with diffuse and direct components) have not been established. To provide reproducible determinations for these concentrators is critical. Finally, there is a very significant need in the area of concentrator measurements to determine the quantum efficiencies of devices under the concentrated light conditions. 
This is a non-trivial problem, which is exacerbated at higher concentrations and for multiplejunction concentrator technologies.

New technologies. New device types, such as thermophotovoltaics, dye-sensitized and electrochemical cells, quantum-dots/porous, material/organic semiconductors, and a host of other next-generation technologies not yet reported, need attention for performance determinations. Spectral effects, response times, and illumination response are critical, and methods for ensuring these must be considered and adequately addressed. Criteria for these device technologies might have been substantially different, and imposition of "current" test and measurement formalities could be erroneous.

Artifacts. Sensitivities and measurement artifacts are always concerns for the measurement scientist. For new device types, these must be probed and identified before standard measurements are adopted. For these and existing technologies (e.g., $\mathrm{Cu}(\mathrm{In}, \mathrm{Ga})(\mathrm{S}, \mathrm{Se})_{2}$, $\mathrm{CdTe}$, a-Si:H), stabilization criteria must be established. Changes with light exposure are certainly concerns; whether a device diminishes in performance upon exposure or whether it improves! Area is a known problem for research $\left(<1 \mathrm{~cm}^{2}\right.$ areas) devices in which collection from outside the "defined" area is significant, area definition is complicated by ragged or less defined edges, and probe contacts have reliability and shading problems. Area is currently the most common source of error for research devices. For larger area device measurements, accuracy is still a concern. Pulsed-light simulators used for modules in both development and in manufacturing environments may have problems with uniformity and accurate spectral determinations. Capacitive and other transient effects in the module can hinder measurement accuracy. Large-area continuous simulators are now in operation that can alleviate some of these problems and permit the application of spectral correction methods used in cellefficiency measurements. Module ratings and measurement methodologies (e.g., power vs. efficiency, temperature, etc.) must also be addressed to better meet the needs of the industry and the client.

In-situ and smart diagnostics. Characterization will become more integrated into the research equipment and into the manufacturing environment. To realize thin-film technologies with greater than $20 \%$ efficiencies and multiple-junction thin-film technologies with greater than $25 \%$, for example, it will be required to evaluate the surfaces of materials as they grow in order to exactly engineer their properties. In the manufacturing facility, the lines will become more automated, and materials and device performance will have to be evaluated during processing to ensure yields and manufacturing cost effectiveness. Non-contact evaluation methods will become critical. Performance evaluation and other techniques will have to be developed to meet these manufacturing criteria.

For long-term needs (10 years and out), performance measurements and characterization have much in common with other technology requirements - they have to be responsive to technology directions and development. The coming PV Industry Roadmap should serve as a useful document in guiding planning for characterization efforts, much like the Semiconductor Roadmap provided the electronics industry equipment manufacturers and other stakeholders with a strategy for equipment development and supporting technology requirements. For the next generation of photovoltaics, those technologies that will be R\&D interests after 2010 and commercialized from 2015 through 2025, measurements 
and characterization technologies have to position themselves to be flexible and responsive to the needs. Many of the techniques that need to be used to address the needs of the technologies in this longer period of time will be developed in response to other requirements in the related electronics industries. Others will have to be adapted or specifically developed for these next-generation PV technologies. For performance, some areas will likely include (2006 and beyond):

- Very high concentration devices, with operating environments exceeding 1000 suns and perhaps approaching 5000 suns.

- Multi-junction approaches, with 4-10 junctions possible. This will require techniques that not only evaluate performance parameters for these extremely complex solar cells, but also provide techniques and standards for devices having efficiencies exceeding 50\%.

- Low-bandgap cells, determining parameters for devices down to $0.2 \mathrm{eV}$. The multi-cell problems addressed in the previous bullet apply. Thermophotovoltaics for terrestrial applications might require extending to these low band-gap regimes. Accompanying the problems of low band-gap evaluations will be the system performance determinations that are critical to such technologies.

- Area performance measurements will require both larger areas (for product evaluation, with simulators and techniques capable of perhaps $20 \mathrm{~m}^{2}$ for some large-area technologies.) On the other end of spatial resolution, nanoscale-type performance measurement systems will be needed for research optimization of ultra-small area devices and for determination of localized efficiencies on device surfaces to optimize processing and growth parameters. Such tools will become important for maximizing device operation, linking performance events on the nanoscale to eventual macroscale operations. Nanotechnology will be part of $\mathrm{PV}$, both for device development and for characterization efforts.

- Reliability and durability criteria, both for qualifying these new cell technologies and for ascertaining their operating-lifetime probabilities. This would include efforts at service lifetime predictions and development of acceptable accelerated lifetime tests and procedures.

- Systems, including balance of system components, cannot be neglected. As this PV technology becomes more commonly deployed (especially for the US markets), the system reliability is a major concern. This will call attention to the balance of system components (inverters, charge controllers, batteries). Certainly, their reliability is essential, and will require characterization support to establish parameters. Moreover, storage (next-generation batteries and non-conventional approaches) will become a greater part of the R\&D activity.

- Resource assessment and metrology will become increasingly important. First, the measurement of the illumination sources for simulators will become more complex. With the more complicated designs for cells and spectral matching specifications for devices, the exact and rapid determination of the source power density, spectral distribution, and uniformity will become more critical for standards and other laboratories. Secondly, for the proper deployment and design of systems (especially that generation that we do not even yet know), 
the solar resource determination will become essential. This will mandate the mapping of wide areas of the globe for this solar commodity (e.g., satellite and remote sensing) and having mobile and inexpensive equipment for monitoring these resource parameters "on location". The design of systems will become more locality sensitive, especially with a growing and different portfolio of PV technologies.

The communications revolution will become a partner with performance and all characterization techniques in the $21^{\text {st }}$ century. No longer will the ability "to witness", transfer data, or control systems be confined by the locality. Many laboratories have already embarked on techniques to provide immediate transfer of data. The future will focus on these areas, and control of instrumentation will not require operators in remote locations. As the business of manufacturing becomes global, the cost of remote operations will be minimized by "remote viewing" via a much expanded and dedicated Internet capability to control and measure outside the more distant facility.

It is realized that the more standard techniques of characterization will provide a baseline for PV characterization in a large number of cases. These include SIMS, TEM, Raman, STM, STS, SAXS etc. All of these methods are developments of the research community and many are used for needed characterization of the PV industry. One needs to provide a balance between the fundamental community and those in the private sector. Below are a few examples of new techniques that have been developed in the last few years and applied to the PV studies. It is not meant to be exhaustive but as a useful view into the need for this area of research. One needs to always try and couple the characterization techniques with the various growers to ensure a constant feedback on the various results.

\section{Examples of New Innovative Techniques}

The development of NSOM [1,2] has not only extended optical microscopy beyond the diffraction limit, but has also allowed spectroscopic properties to be measured with submicron resolution. Such resolution is sufficient to provide optical characterization of extended defects and grain boundaries in polycrystalline solar cells. The technique works with a proximal probe: a single mode optical fiber drawn to a fine tip and coated with an opaque metal. The resolution is governed by the size of the aperture formed at the apex of the fiber tip, and it can be an order of magnitude less than the diffraction-limited resolution, as illustrated in Fig. 1.

\section{$\underline{\text { Spatial and Time-Resolved PL and PLE }}$}

Near-field and far-field optics can be used simultaneously, allowing localized photoluminescence (PL) or photoluminescence excitation (PLE) experiments to be carried out (using illumination-mode NSOM or collection-mode NSOM). Using the scanning confocal microscopy technique (here, both the exciting and PL light go through a microscope objective that is used in place of the NSOM tip) one can achieve a spatial resolution of 0.5 $\mu \mathrm{m}$ under special circumstances with the sample at $5 \mathrm{~K}$. For higher spatial resolution $(0.1$ $\mu \mathrm{m})$, the NSOM tip can be used to illuminate the sample, while the far-field lens is used to collect the PL. In this mode, a measure of carrier diffusion to localized traps (e.g., at grain boundaries) may be inferred by examining NSOM images. 
By collecting the PL in far-field, submicron measurements of PLE can be obtained giving additional data on alloy composition and band tailing effects. Alternatively, exciting in far field, PL spectra may be recorded with submicron resolution, and both grain interiors and grain boundaries can be locally probed. The PL can be spectrally resolved, for determining alloy composition and identifying impurities, or time-resolved, for identifying areas of rapid recombination. In ternary alloys, the technique can be used to map the variations in exciton emission wavelength due to compositional fluctuations (Fig. II). For the first time, these measurements can focus directly upon the suspect regions, rather than studying the macroscopic spatially averaged properties of polycrystalline films.
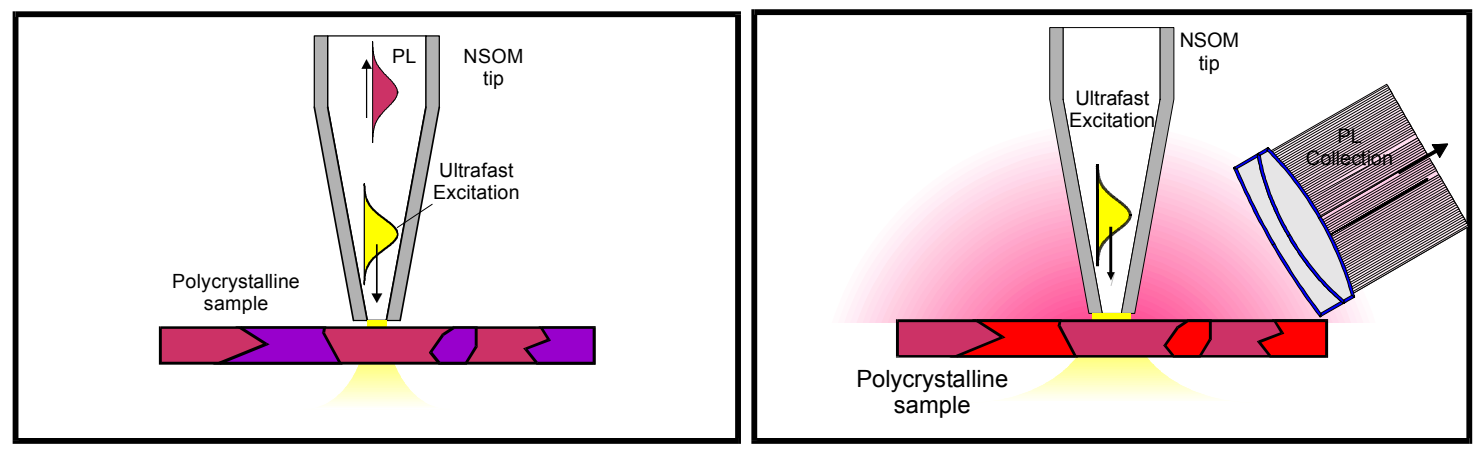

Figure I: (Left) Schematic of the near-field scanning optical microscopy (NSOM) technique showing near-field excitation and collection of light. (Right) Here, the excitation is through the fiber tip whereas the PL is collected by the far-field lens.

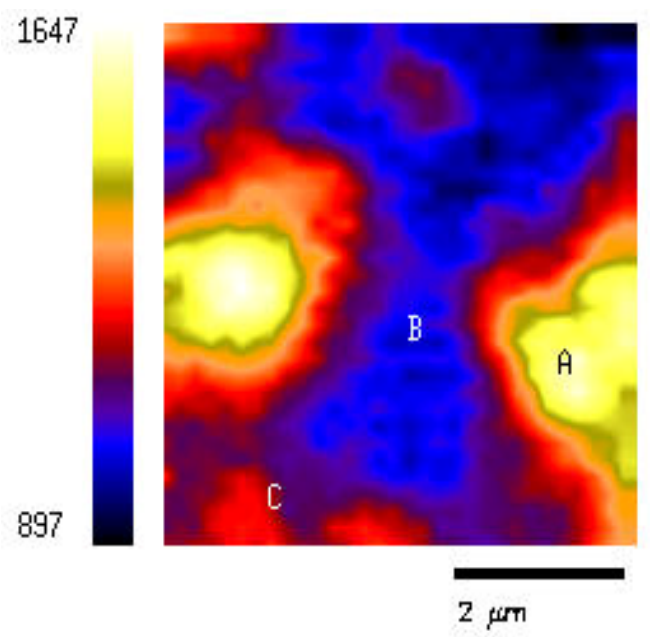

Figure II: Spatially resolved PL of polycrystalline CdTe cell taken at 5K. Points labeled A-C are locations where corresponding spectra showed peak shifts due to variations in sulfur content resulting from interdiffusion and alloying of sulfur with the CdTe (the sulfur diffuses across the heterojunction formed with $\mathrm{CdS}$ in a $\mathrm{CdTe} / \mathrm{CdS}$ solar cell). 
A femtosecond laser system is ideal for time-resolved spectroscopy of PV materials. Tunability is achieved through parametric amplification of a visible-light continuum to provide the necessary broadband probe, with a multichannel CCD detector for parallel measurements of a large spectral region around a semiconductor band edge. The system provides femtosecond pulses through most of the visible and near-infrared spectrum. The femtosecond laser can be coupled into the cryogenic NSOM to enable time-resolved studies to be carried out at submicron spatial resolution (see refs. [3-5]).

\section{Spatially-Resolved Photo-Current Using NSOM}

The highly localized optical excitation provided by NSOM can be used to obtain highresolution images of solar cell efficiency by mapping the collected photocurrent, as the probe is scanned [6]. This is the analogue of electron-beam-induced current in the scanning electron microscope, a well-established technique for mapping electrical properties. However, it has the advantage that one can select the excitation wavelength, and so obtain maps for any desired part of the solar spectrum - again, with sufficient resolution to distinguish single defects within grains or single grain boundaries. Alternatively, with the probe stationary over a specific defect, localized photocurrent spectroscopy can be performed by varying the wavelength of the optical excitation to provide the full absorption spectrum of an individual defect or a grain boundary [7]. Again, the combination of spatial and spectral resolution provided by NSOM provides detailed insight into local cell properties. It demonstrates directly which regions of the cell are less efficient, while the spectral capability provides a diagnostic capability for identifying impurities.

\section{$\underline{\text { Transient Differential Absorption }}$}

By combining the ultrafast time resolution of the laser system with the submicron spatial resolution of the NSOM, one can observe carrier populations at individual crystal grains using the transient differential absorption technique. This is a valuable spectroscopic technique that can reveal the occupation of electron states much more directly than does PL. The femtosecond laser system must be specifically chosen for this type of measurement for materials with bandgaps in the $400 \mathrm{~nm}-1.5 \mu \mathrm{m}$ range. Here, the whitelight continuum is capable of measuring absorption at the entire near-band-edge region simultaneously. This spectral range corresponds to an important energy scale for carrier processes in disordered materials. Of particular interest will be time-resolved vibrational spectroscopic techniques that can probe local vibrational modes associated with the defect centers.

Simple transmission data with submicron spatial resolution is valuable for identifying regions of sulfur diffusion, however, the true potential of this technique is to measure differential absorption. In measuring the change in absorption caused by an intense earlier pulse, the spectral population of photoexcited carriers created by that pulse are observed. The dynamics of this population is time-resolved because of the sampling nature of the experiment, and it is limited only by the 150 -fs pulsewidth of the laser. Low-spatial resolution measurements of this type in CdS [8] have been performed. If one can introduce a limited white-light 
continuum into the NSOM tip and then collect this light after transmission through a specially prepared sample, a spatial and energy map of carrier occupation can be obtained. Since such maps can be obtained at different times in the carrier evolution, information regarding carrier transport in a complex polycrystalline sample can also be obtained.

\section{X-ray Scattering, Fluorescence, and Absorption Methods}

Realizing the tremendous financial commitment invested in synchrotron sources over the past two decades by DOE, one should take advantage of this investment. X-rays from synchrotron radiation provide convenient tools for characterizing microstructures, interfaces, impurities, atomic-density profiles, and defects in PV materials. For the purpose of investigating these microscopic short-range structures, the conventional x-ray diffraction method is not useful. Recent progress in short-range probing techniques, such as grazing incidence x-ray scattering (GIXS), angular dependence of x-ray fluorescence (ADXRF), and $\mathrm{x}$-ray absorption fine structures (XAFS) has made it possible to obtain element-specific information about various types of PV materials, especially the thin-film junctions that are widely recognized to be extremely important for the development of next-generation PV devices.

The angular dependence of x-ray scattering and fluorescence is especially useful tools for investigating the interface morphology of multilayer materials [9-21]. Measurements of GIXS and ADXRF can afford a nondestructive approach to obtain important microstructural information about the buried interfaces, such as the layer thickness, interfacial roughness, and correlation lengths of height fluctuations. Through a control of the x-ray probing depth and field distribution by varying the wavelength and incidence angle, the depth profile of a selected atomic species in the layer structure can be obtained. X-ray fluorescence and anomalous scattering also allow an element-specific method to probe the impurity location and intermixing of constituent atoms in the interfacial region. Theoretical analysis of $x$-ray propagation in layer structures, as well as methods used for obtaining interfacial roughness and correlation lengths can be found in [15] and references cited herein. It should be emphasized that the usual determination of rms roughness is insufficient to characterize a random interface; two rough surfaces with totally different rates of height fluctuations along the surface could have the same value of rms roughness. It must at least be complemented by specifying the correlation lengths in order to properly define an actual rough interface.

XAFS generally refers to extended x-ray absorption fine structure (EXAFS) and near-edge xray absorption fine structure (NEXAFS or XANES). These spectra can be used to obtain valuable atomic-scale information on the local structure and chemical states of a specific atomic species in multicomponent materials, such as interatomic distance, coordination number, and local disorder. Detailed descriptions of the XAFS techniques can be found in several review articles [22-23].

\section{Positron Annihilation Spectroscopy}

Of greater significance are atomic-sized defects, which degrade the electronic properties of $a$-Si. The dominant type is an amphoteric Si dangling bond, labeled $D$ that can trap up to 2 electrons, which determine its charge. In intrinsic $a-\mathrm{Si}: \mathrm{H}$ the uncharged variety 
prevails. The negatively charged state, $\mathrm{D}^{-}$-- occupied by 2 electrons - is most common in $n$-doped $a$-Si:H.

Recently, new results from positron beams have also helped answer fundamental questions regarding amorphous Si. Direct experimental confirmation and theoretical modeling was obtained with positron (antimatter to electron) annihilation spectroscopy (PAS). Positrons are attracted and trapped by negatively charged defects in materials, and eventually annihilate with an electron sitting in or near the trap. With a sophisticated coincidence detection system, the Doppler broadening of the annihilation radiation is used to "fingerprint" the chemical element type of atoms next to the defects. In combination with a theoretical model phosphorous was identified for the first time by comparing P-implanted and P-doped samples to samples without phosphorous.

The background of figure III depicts a two-dimensional raw data set from the P implanted sample. The comparison (i.e., ratio) of the data and data of the P-doped sample to the reference samples is shown in the overlay. A broad component due to conduction band electrons was taken out of the spectrum. The phosphorous "fingerprint" appears as a line at 1.3 atomic units. This directly correlates with the theoretical model of positron trapping and annihilating at the $\mathrm{P}$ decorated dangling bond site ${ }^{*} \mathrm{D}^{-}$. The basic atomic structure of a-Si and the positron wave function around a dangling-bond $\mathrm{Si}$ in the center where the wave function is enhanced by $10 \%$ are shown in figure IV.

Now that the charge-trapping site has been identified, research can focus on the reduction of the $\mathrm{P}$ content in solar cells and the number of dangling bond sites. With the positron technique, the $\mathrm{P}$ content can be monitored for the first time.

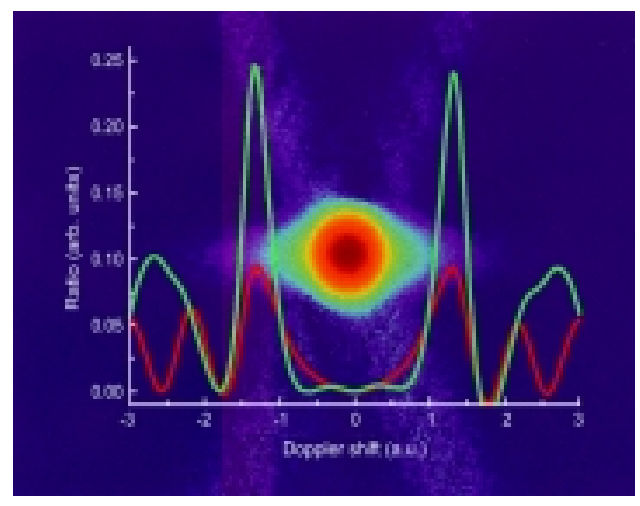

Figure III

Figure III Background: A 2-dimensional positron annihilation spectrum of P-implanted $a$ $\mathrm{Si}$. The two annihilation quanta are registered in coincidence and their difference in energy (Doppler shift) is plotted versus their sum energy. Accumulated counts in each bin are color coded with blue corresponding to 1 and red to $10^{5}$ counts. Foreground: The Doppler broadening information extracted from the raw 2D data in ratio to the Doppler data from a phosphor-free sample. The P-implanted sample data are in green and the Pdoped data are in red. The P-"fingerprint" appears at 1.3 atomic units. The data are 
symmetric about 0 momentum. A broad component due to conduction band electrons was subtracted.

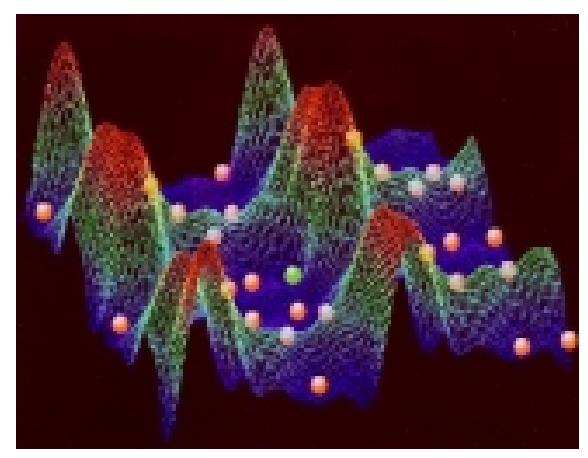

Figure IV

Figure IV: A 3D representation of an atomic model of $a$-Si (red spheres). Drawn is a section through a $4 \times 4 \times 2$ super cell where a $\mathrm{Si}$ atom with a dangling bond is located in the center (in green). Drawn round the atoms is the positron wave function (high probability in dark red). The most likely place for the positron is near the dangling bond Si.

\section{REFERENCES}

1. E. Betzig and J. K. Trautman, Science, 257, 189 (1992).

2. H. Heinzelmann and D. W. Pohl, Appl. Phys., A 59, 89 (1994).

3. S. Smith, N. C. R. Holme, B. G. Orr, R. Kopelman, and T. B. Norris, Ultramicroscopy, 71, 213 (1998).

4. S. Smith, PhD Thesis, University of Michigan, 1996.

5. S. Smith, B. G. Orr, R. Kopelman, and T .B. Norris, Ultramicroscopy, 57, 173 (1995).

6. A. A. McDaniel, J. W. P. Hsu, and A. M. Gabor, Appl. Phys. Lett., 70, 3555 (1997).

7. A. Richter, J. W. Tomm, and Ch. Lienau, Appl. Phys. Lett., 69, 3981 (1996).

8. D. H. Levi, B. D. Fluegel, R. K. Ahrenkiel, A. D. Compaan and L M. Woods, Photovoltaic Specialists Conference of IEEE Twenty-Five, Washington, DC. NREL/TP410-21091, p. 69 (1996).

9. L.G. Parratt, Phys. Rev., 35, 359 (1954).

10. B. Vidal and P. Vincent, Appl. Optics, 23, 1794 (1984).

11. A. Krol, C. Sher, and Y.H. Kao, Phys. Rev. B38, 8579 (1988).

12. D.K.G. de Boer, Phys. Rev., B44, 498 (1991).

13. S.K. Sinha, E.B. Sirota, S. Garott, and H.B. Stanley, Phys. Rev., B38, 2297 (1988).

14. M.K. Sanyal, S.K. Sinha, A. Gibaud, K.G. Huang, B.L. Carvalho, M. Rafailovich, J. Sokolov, X. Zhao, and W. Zhao, Europhys. Lett., 12, 691 (1993).

15. Z.H. Ming, A. Krol, Y.L. Soo, Y.H. Kao, J.S. Park, and K.L. Wang, Phys. Rev., B47, 16373 (1993). 
16. Z.H. Ming, Y.L. Soo, S. Huang, Y.H. Kao, J.C. Tsang, and S.S. Iyer, Appl. Phys. Lett., 65, 1382 (1994).

17. Z.H. Ming, S. Huang, Y.L. Soo, Y.H. Kao, T. Carns, and K.L. Wang, Appl. Phys. Lett., 67, 629 (1995).

18. Z.H. Ming, Y.L. Soo, S. Huang, Y.H. Kao, K. Stair, G. Devane, and C. Choi-Feng, Appl. Phys. Lett., 66, 165 (1995).

19. S. Huang, Z.H. Ming, Y.L. Soo, Y.H. Kao, M. Tanaka, and H. Munekata, J. Appl. Phys. 79, 1435 (1996).

20. Y.L. Soo, S. Huang, Y.H. Kao, and A.D. Compaan, J. Appl. Phys., 83, 4173 (1998).

21. Y.L. Soo, S. Huang, Y.H. Kao, and A.D. Compaan, Appl. Phys. Lett., 74, 218 (1999).

22. P.A. Lee, P.H. Citrin, P. Eisenberger, and B.M. Kincaid, Rev. Mod. Phys., 53, 760 (1981).

23. D.E. Sayer and B.A. Bunker, in X-ray Absorptions, edited by D.C. Koningberger and R. Prin (Wiley, New York, 1988) pp. 211 and 231. 


\section{REPORT DOCUMENTATION PAGE}

Form Approved

OMB NO. 0704-0188

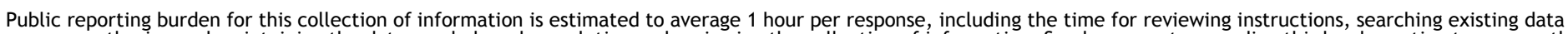

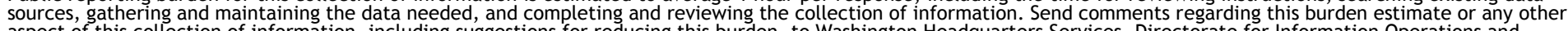

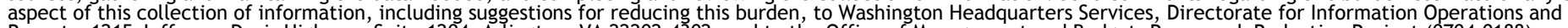

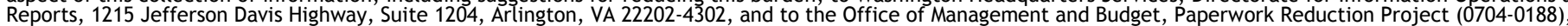
Washington, DC 20503

\begin{tabular}{|l|c|c|}
\hline 1. AGENCY USE ONLY (Leave blank) & $\begin{array}{l}\text { 2. REPORT DATE } \\
\text { August 1999 }\end{array}$ & $\begin{array}{l}\text { 3. REPORT TYPE AND DATES COVERED } \\
\text { Workshop proceedings }\end{array}$ \\
\hline 4. TITLE AND SUBTITLE &
\end{tabular}

Workshop on Basic Research Opportunities in Photovoltaics

6. AUTHOR(S)

Editors: John Benner, Satyen Deb, and Robert McConnell

5. FUNDING NUMBERS

$\mathrm{C}$

TA: PV902601

8. PERFORMING ORGANIZATION REPORT NUMBER

9. SPONSORING/MONITORING AGENCY NAME(S) AND ADDRESS(ES) National Renewable Energy Laboratory

1617 Cole Blvd.

Golden, CO 80401-3393

10. SPONSORING/MONITORING AGENCY REPORT NUMBER

BK-590-26952

11. SUPPLEMENTARY NOTES

12a. DISTRIBUTION/AVAILABILITY STATEMENT 12b. DISTRIBUTION CODE

National Technical Information Service

U.S. Department of Commerce

5285 Port Royal Road

Springfield, VA 22161

13. ABSTRACT (Maximum 200 words)

The Basic Research Opportunities in Photovoltaics Workshop was held on May 3, 1999, in Seattle, Washington, in conjunction with the $195^{\text {th }}$ Meeting of the Electrochemical Society. The workshop was structured into eight topics. Each topic area opened with a presentation in which the participants were asked to address the following: a brief introduction of the area of research; key research issues that were identified in an earlier workshop in 1992; what fundamental research has been done since then or is currently being done to address those issues; what are the research issues that are still relevant in light of advances made since the first workshop; identification of new fundamental research opportunities that will lead to important advances and innovations; and identification of significant commonalities and common research issues that have a cross-cutting impact, such as logically exist in silicon-based thin films, II-VI, and related materials. The topic areas discussed included amorphous and microcrystalline silicon, crystalline silicon, cadmium telluride, copper indium diselenide; III-V materials; novel materials and energy conversion approaches, semiconducting oxides, and characterization. After the meeting, participants in each working topic continued discussions by electronic means, completing journal articles that are to be published as a separate section in the ECS Proceedings of the "PV for the $21^{\text {st }}$ Century" Symposium.

\section{SUBJECT TERMS}

photovoltaics ; basic research ; amorphous silicon ; micro-crystalline silicon ; crystalliine silicon ; cadmium telluride ; copper indium diselenide ; III-V materials ; novel materials ; energy conversion ; semiconducting oxides ; characterization

17. SECURITY CLASSIFICATION OF REPORT

Unclassified
18. SECURITY CLASSIFICATION OF THIS PAGE Unclassified
19. SECURITY CLASSIFICATION OF ABSTRACT Unclassified
15. NUMBER OF PAGES 108

16. PRICE CODE

20. LIMITATION OF ABSTRACT

UL 